\title{
Alkaline-earth derivatives of diphenylphosphine-borane
}

\author{
Louis J. Morris, ${ }^{a}$ Michael S. Hill,, ${ }^{\star}$ a Mary F. Mahon, ${ }^{\star, a}$ Ian Manners ${ }^{\star, b}$ and Brian O. \\ Patrick $^{\mathrm{C}}$ \\ ${ }^{a}$ Department of Chemistry, University of Bath, Claverton Down, Bath, BA2 7AY UK; ${ }^{b}$ Department of \\ Chemistry, University of Victoria, Victoria, British Columbia V8P 5C2, Canada; 'c Department of \\ Chemistry, University of British Columbia, 2036 Main Mall, Vancouver, British Columbia V6T 1Z1, \\ Canada.
}

\section{Supplementary information}

\section{X-ray crystallography}

X-ray crystallographic data for compounds 6-9, was collected on a SuperNova, EosS2 diffractometer with the crystals kept at $150.00(10) \mathrm{K}$ during data collection. For compound 10, a Bruker APEX-II CCD diffractometer was used and the crystal kept at 100(2) K. Using Olex2, ${ }^{10}$ all structures were solved with the ShelXT ${ }^{1}$ structure solution program using Intrinsic Phasing and refined with the ShelXL ${ }^{2}$ refinement package using Least Squares minimization.

Correctly resolving a model for the structure of $6 \mathbf{a}$ presented this crystallographer with something of a conundrum as well as shortening her life! While the crystal was of good quality (rather than spectacular), the data frames were certainly above average quality. Metrically, an orthorhombic $\mathrm{C}$ type was a possibility but the arising solution was poor and accompanied by a despicable $R$ (int). After some 150 various refinements, all roads led back to the original solution in space group $P 2_{1} / n$. However, in treating the data as arising from a single crystal rendered afforded a refinement that was accompanied by in excess of 130 systematic absence violations - mostly related to the $n$-glide - which could not be neglected. Scrutiny of the raw data suggested the potential for pseudo-merohedral twinning of the sample to be present but efforts to resolve this at the point of integration were unsuccessful (probably because of the degree of overlap). Ultimately, the data were integrated as arising from a single component, and the twinning (approx 9\%) was addressed 'after the fact' i.e. during convergence of the model. The lattice in this structure contains a very high level of solvent, for which the electron density was smeared and disorder was prevalent. On balance, a heavily parameterized disorder-model treatment was abandoned in favor of using a solvent mask. An allowance for 4 molecules of toluene, per asymmetric unit, has been made in the model as presented to account for the electron density evidence prior to invoking the solvent mask algorithm.

Boron bound hydrogens were located and refined freely in the structure of $\mathbf{6 b}$. The asymmetric unit hosts one molecule of toluene in addition to one molecule of the magnesium complex.

The boron-bound hydrogen atoms in $\mathbf{7 b}$ were located and refined without restraints. C31 was modelled to take account of 80:20 disorder. Chemically equivalent $\mathrm{C}-\mathrm{C}$ distances involving the arising fractional occupancy carbons were restrained to being similar in the final least-squares cycles.

In compound 8, the asymmetric unit was seen to contain 1.5 molecules of guest toluene in addition to one molecule of the main feature. The hydride ligand in the latter, plus the hydrogens attached to $B 1$, were readily located and refined without restraints. Both solvent moieties were disordered. In particular, the full toluene based on C78, was refined over 2 proximate sites in a 55:45 ratio. The second toluene moiety, based on $\mathrm{C} 71$, is disordered with itself and is present in the asymmetric unit at half site-occupancy. Distance and ADP restraints 
were included in the disordered regions, to assist convergence, and rings were treated as rigid hexagons.

The asymmetric unit in $\mathbf{9}$ comprises one molecule of the calcium containing trimer, one ordered molecule of toluene (based on C120), $1 / 2$ of one molecule of toluene (based on C8S) and 3 other regions of solvent. The boron bound hydrogens and the hydride ligand, in the main feature, were all readily located, and refined without restraints. Atoms C8S and C11S (in the $1 / 2$ toluene molecule were found to be coincident with a 2 -fold rotation axis intrinsic to the space group. This necessarily means that the methyl group therein exhibits 50:50 crystallographic disorder about this symmetry element; hence, C14S is present at half site-occupancy in the asymmetric unit. The unresolved regions of solvent were treated using the solvent mask algorithm available in Olex-2. It was evident that these regions amounted to 2.5 molecules of toluene, albeit disordered in all cases. Consequently, an allowance for these 2.5 lattice guest has been made in the formula as presented.

The asymmetric unit in the structure of compound 10 contains two crystallographically independent dimer halves and two regions of solvent. Each dimer can be completed by virtue of inversion symmetry which is present in the space group. The boron-bound hydrogen atoms were located and refined without restraints. The first of the two solvent regions was modelled as a full molecule of toluene, disordered over two proximate sites, in a 55:45 ratio. ADP restraints were applied in this region. The second solvent region straddles a crystallographic inversion centre which necessarily means that disorder is present. Ultimately the motif in the asymmetric unit was modelled as a toluene moiety, with half site occupancy, in which the methyl group was disordered over two sites in a 35:15 ratio. The fractional occupancy hydrogen atoms which were disordered with this methyl functionality were tentatively assigned and refined with restraints. 
Table S1: X-ray crystallographic analysis.

\begin{tabular}{|c|c|c|c|c|c|c|}
\hline dentification code & $6 a$ & $6 b$ & $7 b$ & 8 & 9 & 10 \\
\hline Empirical formula & $\begin{array}{l}\mathrm{C}_{110} \mathrm{H}_{165} \mathrm{~B}_{2} \mathrm{Mg}_{2} \\
\mathrm{~N}_{4} \mathrm{P}_{2}\end{array}$ & $\begin{array}{l}\mathrm{C}_{52} \mathrm{H}_{70} \mathrm{BMgN} \\
{ }_{2} \mathrm{OP}\end{array}$ & $\begin{array}{l}\mathrm{C}_{139} \mathrm{H}_{181} \mathrm{~B}_{2} \mathrm{Ca}_{3} \\
\mathrm{~N}_{6} \mathrm{P}_{2}\end{array}$ & $\begin{array}{l}\mathrm{C}_{80.5} \mathrm{H}_{108} \mathrm{BMg} \\
{ }_{2} \mathrm{~N}_{4} \mathrm{P}\end{array}$ & $\begin{array}{l}\mathrm{C}_{45} \mathrm{H}_{62} \mathrm{BCaN} \\
{ }_{2} \mathrm{OP}\end{array}$ & $\begin{array}{l}\mathrm{C}_{92.5} \mathrm{H}_{126} \mathrm{~B}_{4} \mathrm{Mg}_{2} \\
\mathrm{~N}_{4} \mathrm{P}_{2}\end{array}$ \\
\hline Formula weight & 1675.63 & 805.19 & 2139.69 & 1222.10 & 728.82 & 1447.77 \\
\hline Temperature/K & $150.00(10)$ & $150.00(10)$ & $149.9(3)$ & $150.00(10)$ & $150.0(3)$ & $100(2)$ \\
\hline Crystal system & monoclinic & nonoclinic & monoclinic & triclinic & triclinic & triclinic \\
\hline Space group & $\mathrm{P} 2_{1} / \mathrm{n}$ & $\mathrm{P} 2_{1} / \mathrm{c}$ & $\mathrm{C} 2 / \mathrm{c}$ & $P-1$ & $P-1$ & $P-1$ \\
\hline $\mathrm{a} / \AA$ & $18.8895(2)$ & $12.3443(2)$ & $24.9783(5)$ & 13.1986(3) & $11.8835(3)$ & $13.4773(6)$ \\
\hline $\mathrm{b} / \AA ̊$ & $16.2536(2)$ & $41.1065(7)$ & 17.9983(3) & $13.8418(3)$ & $12.2918(3)$ & $15.8128(8)$ \\
\hline$c / \AA ̊ A$ & $34.1206(5)$ & $9.5729(2)$ & $58.5938(8)$ & $20.1666(3)$ & $15.4936(4)$ & $22.6902(11)$ \\
\hline$\alpha /^{\circ}$ & 90 & 90 & 90 & $95.509(2)$ & $82.120(2)$ & $104.5820(10)$ \\
\hline$\beta /^{\circ}$ & $105.7783(13)$ & $90.692(2)$ & $100.806(2)$ & $93.735(2)$ & $74.020(2)$ & $92.9400(10)$ \\
\hline $\mathrm{Y} /{ }^{\circ}$ & 90 & 90 & 90 & $98.781(2)$ & $83.995(2)$ & $109.9620(10)$ \\
\hline Volume/ $/ \AA^{3}$ & $10081.1(2)$ & $4857.23(15)$ & $25874.7(8)$ & $3612.34(13)$ & $2149.83(10)$ & $4348.3(4)$ \\
\hline Z & 4 & 4 & 8 & 2 & 2 & 2 \\
\hline$\rho_{\text {calc }} \mathrm{g} / \mathrm{cm}^{3}$ & 1.104 & 1.101 & 1.099 & 1.124 & 1.126 & 1.106 \\
\hline$\mu / \mathrm{mm}^{-1}$ & 0.863 & 0.898 & 1.713 & 0.838 & 1.857 & 0.110 \\
\hline$F(000)$ & 3668.0 & 1744.0 & 9256.0 & 1326.0 & 788.0 & 1566.0 \\
\hline Crystal size $/ \mathrm{mm}^{3}$ & $\begin{array}{l}0.317 \times 0.189 \\
\times 0.028\end{array}$ & $\begin{array}{l}0.399 \\
0.134 \\
0.098\end{array}$ & $\begin{array}{l}0.166 \times 0.145 \\
\times 0.073\end{array}$ & $\begin{array}{l}0.143 \\
0.073 \\
0.055\end{array}$ & $\begin{array}{l}0.163 \\
0.093 \\
0.072\end{array}$ & $\begin{array}{l}0.27 \times 0.23 \times \\
0.11\end{array}$ \\
\hline Radiation & $\begin{array}{l}\text { CuKa }(\lambda= \\
1.54184)\end{array}$ & $\begin{array}{l}\text { CuKa }(\lambda= \\
1.54184)\end{array}$ & $\begin{array}{l}\text { CuKa }(\lambda= \\
1.54184)\end{array}$ & $\begin{array}{l}\text { CuKa }(\lambda= \\
1.54184)\end{array}$ & $\begin{array}{l}\text { CuKa }(\lambda= \\
1.54184)\end{array}$ & $\begin{array}{l}\text { MoKa } \\
0.71073)\end{array}(\lambda=$ \\
\hline $\begin{array}{l}2 \Theta \text { range for data } \\
{\text { collection } /{ }^{\circ}}^{\circ}\end{array}$ & $\begin{array}{ll}6.068 & \text { to } \\
146.942 & \end{array}$ & $\begin{array}{lr}7.162 \\
146.734\end{array}$ to & $\begin{array}{ll}6.09 & \text { to } \\
146.492 & \end{array}$ & $\begin{array}{ll}6.498 & \text { to } \\
147.196 & \end{array}$ & $\begin{array}{l}5.972 \\
146.948\end{array}$ to & 2.86 to 61.16 \\
\hline Index ranges & $\begin{array}{l}-23 \leq h \leq 20 \\
20 \leq k \leq 19 \\
41 \leq 1 \leq 42\end{array}$ & $\begin{array}{l}-15 \leq h \leq 15 \\
-50 \leq k \leq 50 \\
-7 \leq l \leq 11\end{array}$ & $\begin{array}{l}-29 \leq \mathrm{h} \leq 30 \\
15 \leq \mathrm{k} \leq 22 \\
69 \leq \mathrm{I} \leq 72\end{array}$ & $\begin{array}{l}-16 \leq \mathrm{h} \leq 16,- \\
17 \leq \mathrm{k} \leq 17,- \\
19 \leq \mathrm{I} \leq 25\end{array}$ & $\begin{array}{l}-12 \leq \mathrm{h} \leq 14 \\
-15 \leq \mathrm{k} \leq 15 \\
-19 \leq \mathrm{I} \leq 18\end{array}$ & $\begin{array}{l}-19 \leq \mathrm{h} \leq 19 \\
22 \leq \mathrm{k} \leq 22 \\
31 \leq \mathrm{I} \leq 32\end{array}$ \\
\hline $\begin{array}{l}\text { Reflections } \\
\text { collected }\end{array}$ & 133709 & 62007 & 77627 & 46467 & 25453 & 104431 \\
\hline $\begin{array}{l}\text { Independent } \\
\text { reflections }\end{array}$ & $\begin{array}{l}20547\left[R_{\text {int }}=\right. \\
0.0641, R_{\text {sigma }} \\
=0.0355]\end{array}$ & $\begin{array}{l}9685\left[\mathrm{R}_{\text {int }}=\right. \\
0.0491, \\
\mathrm{R}_{\text {sigma }}= \\
0.0275]=\end{array}$ & $\begin{array}{l}24925\left[R_{\text {int }}=\right. \\
0.0329, R_{\text {sigma }} \\
=0.0409]\end{array}$ & $\begin{array}{l}14438\left[R_{\text {int }}=\right. \\
0.0447, R_{\text {sigma }} \\
=0.0448]\end{array}$ & $\begin{array}{l}8620\left[R_{\text {int }}=\right. \\
0.0243, \\
R_{\text {sigma }}= \\
0.0247]=\end{array}$ & $\begin{array}{l}26663\left[R_{\text {int }}=\right. \\
0.0388, R_{\text {sigma }} \\
=0.0413]\end{array}$ \\
\hline $\begin{array}{l}\text { Data/restraints/para } \\
\text { meters }\end{array}$ & $20547 / 0 / 878$ & $9685 / 0 / 546$ & $24925 / 0 / 1277$ & $\begin{array}{l}14438 / 264 / 8 \\
89\end{array}$ & $8620 / 2 / 491$ & $\begin{array}{l}26663 / 282 / 10 \\
85\end{array}$ \\
\hline $\begin{array}{l}\text { Goodness-of-fit on } \\
\mathrm{F}^{2}\end{array}$ & 1.031 & 1.044 & 1.041 & 1.022 & 1.030 & 1.032 \\
\hline $\begin{array}{l}\text { Final } R \text { indexes } \\
{[\mid>=2 \sigma(I)]}\end{array}$ & $\begin{array}{l}\mathrm{R}_{1}=0.0738 \\
\mathrm{wR}_{2}=0.2127\end{array}$ & $\begin{array}{l}\mathrm{R}_{1}=0.0543 \\
\mathrm{wR}_{2} \\
0.1404\end{array}$ & $\begin{array}{l}R_{1}=0.0536 \\
w R_{2}=0.1204\end{array}$ & $\begin{array}{l}\mathrm{R}_{1}=0.0452, \\
\mathrm{wR}_{2}=0.1115\end{array}$ & $\begin{array}{l}\mathrm{R}_{1}=0.0322, \\
\mathrm{wR}_{2} \\
0.0819\end{array}$ & $\begin{array}{l}\mathrm{R}_{1}=0.0516 \\
\mathrm{wR}_{2}=0.1279\end{array}$ \\
\hline $\begin{array}{l}\text { Final } R \text { indexes [all } \\
\text { data] }\end{array}$ & $\begin{array}{l}R_{1}=0.0816 \\
w R_{2}=0.2209\end{array}$ & $\begin{array}{l}\mathrm{R}_{1}=0.0580, \\
\mathrm{wR}_{2}= \\
0.1443\end{array}$ & $\begin{array}{l}R_{1}=0.0659 \\
w R_{2}=0.1275\end{array}$ & $\begin{array}{l}\mathrm{R}_{1}=0.0591 \\
\mathrm{wR}_{2}=0.1198\end{array}$ & $\begin{array}{l}\mathrm{R}_{1}=0.0348, \\
\mathrm{wR}_{2} \quad= \\
0.0838\end{array}$ & $\begin{array}{l}\mathrm{R}_{1}=0.0768 \\
\mathrm{wR}_{2}=0.1424\end{array}$ \\
\hline $\begin{array}{l}\text { argest diff. } \\
\text { eak/hole / e } \AA^{-3}\end{array}$ & $0.53 /-0.47$ & $0.64 /-0.32$ & $0.38 /-0.33$ & $0.42 /-0.38$ & $0.26 /-0.26$ & $1.26 /-0.57$ \\
\hline
\end{tabular}




\section{NMR spectra}

\section{Compound 6a}

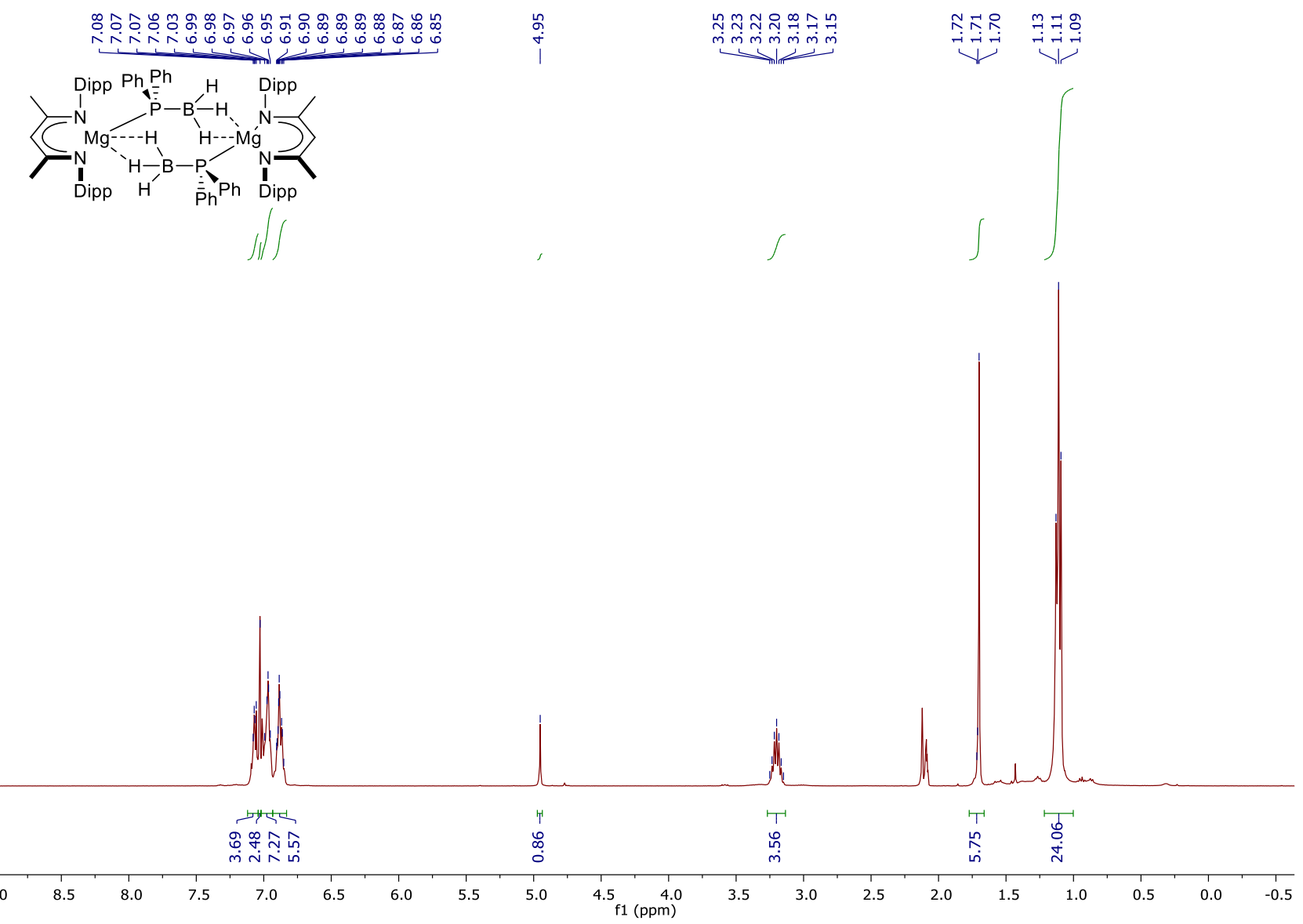

Figure S1: ${ }^{1} \mathrm{H}$ NMR spectrum (400 MHz, $349 \mathrm{~K}, \mathrm{~d}_{8}$-toluene) of compound $\mathbf{6 a}$. 


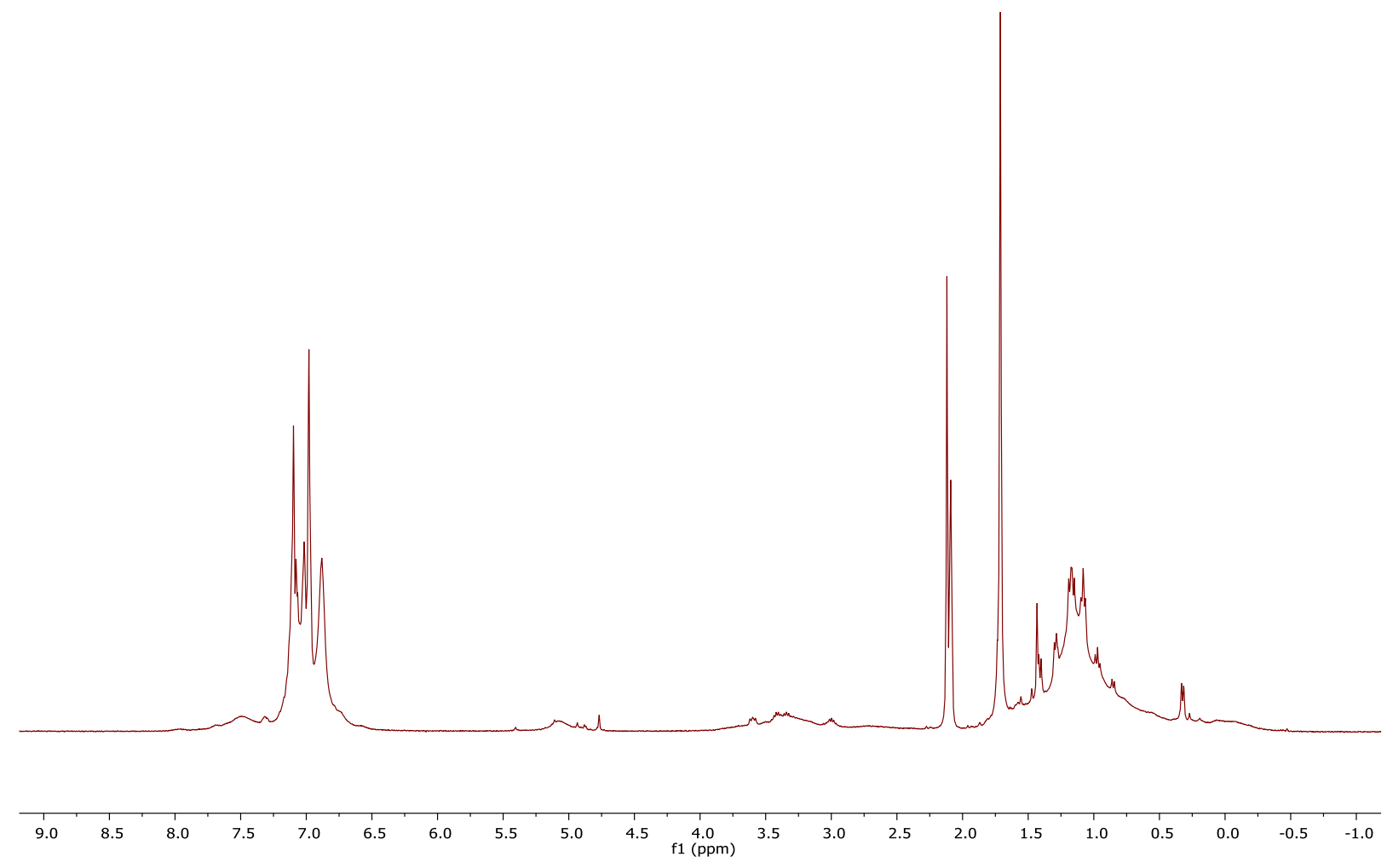

Figure S2: ${ }^{1} \mathrm{H}$ NMR spectrum (400 MHz, $298 \mathrm{~K}, \mathrm{~d}_{8}$-toluene) of compound $\mathbf{6 a}$.

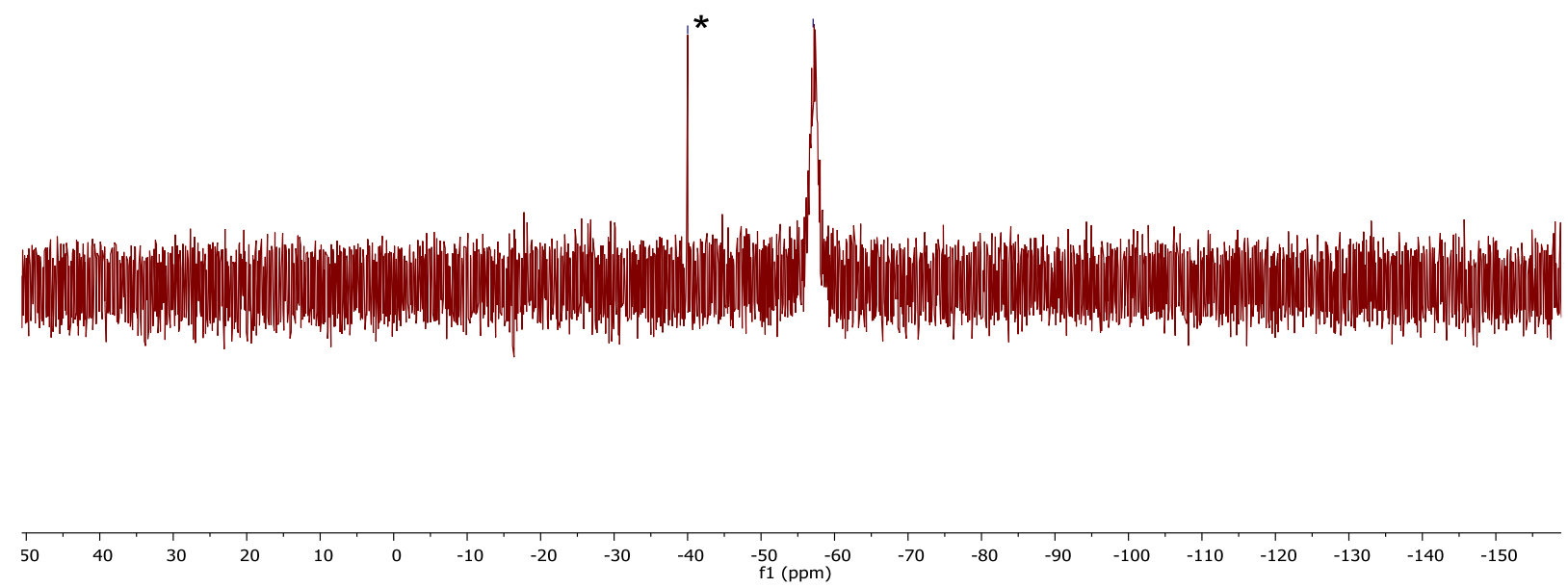

Figure S3: ${ }^{31} \mathrm{P}\left\{{ }^{1} \mathrm{H}\right\}$ NMR spectrum (162 MHz, $349 \mathrm{~K}$ d8-toluene) of compound 6a. * trace diphenylphosphine impurity. 


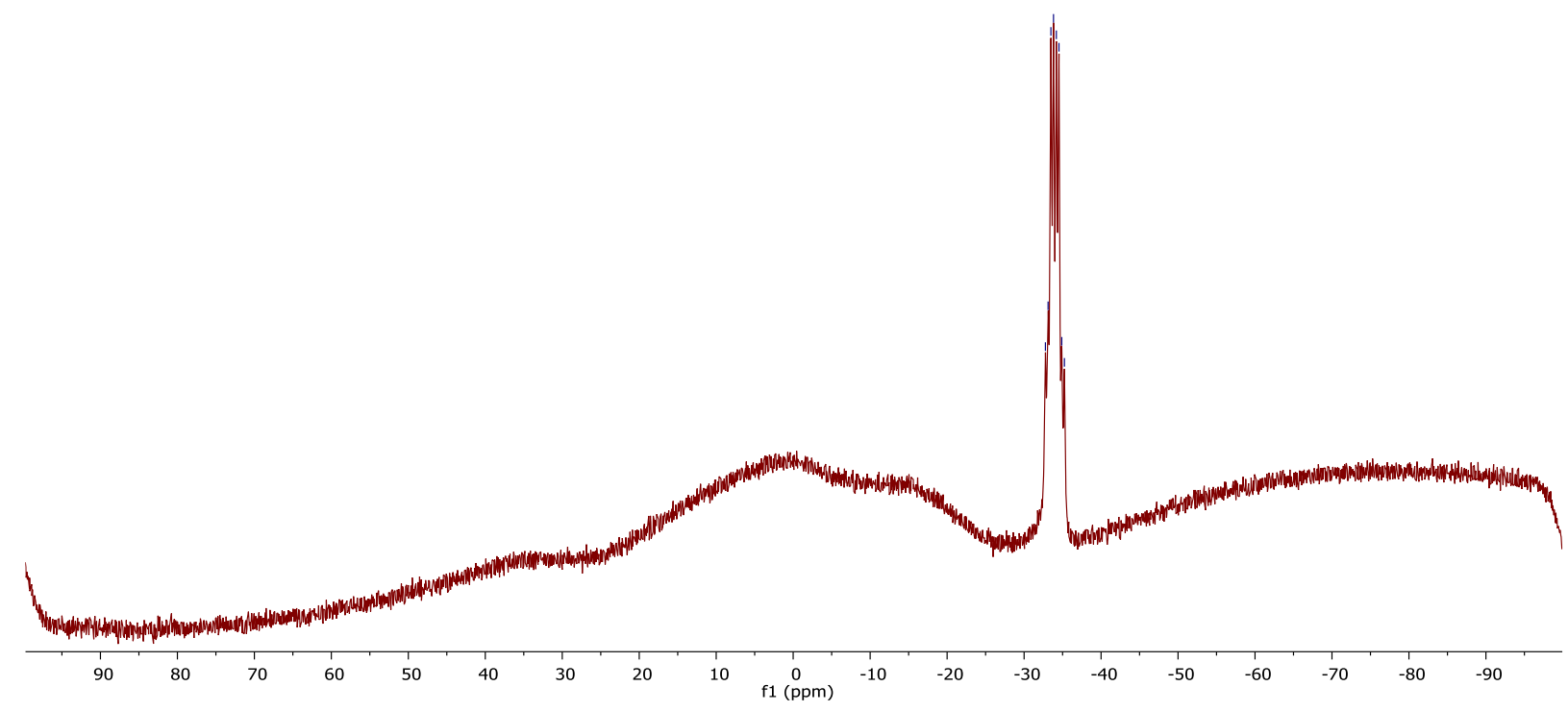

Figure S4: ${ }^{11} \mathrm{~B}$ NMR spectrum (128 MHz, $349 \mathrm{~K}$, d8-toluene) of compound $\mathbf{6 a}$.
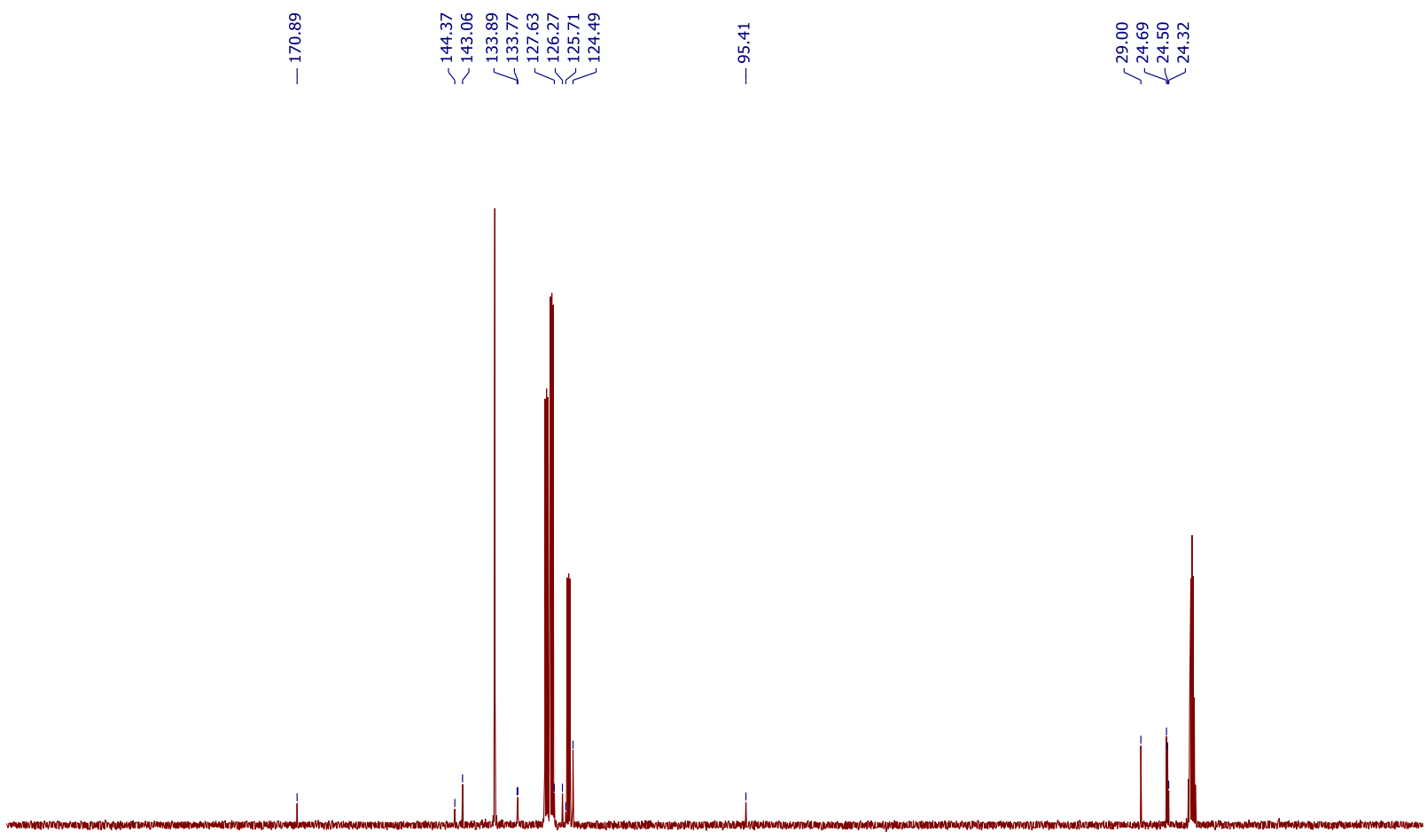

$\begin{array}{lllllllllllllllllllllll}210 & 200 & 190 & 180 & 170 & 160 & 150 & 140 & 130 & 120 & 110 & 100 & 90 & 80 & 70 & 60 & 50 & 40 & 30 & 20 & 10 & 0 & -10\end{array}$

Figure S5: ${ }^{31} \mathrm{C}\left\{{ }^{1} \mathrm{H}\right\}$ NMR spectrum (101 MHz, $349 \mathrm{~K}$, d8-toluene) of compound $6 \mathbf{a}$. 


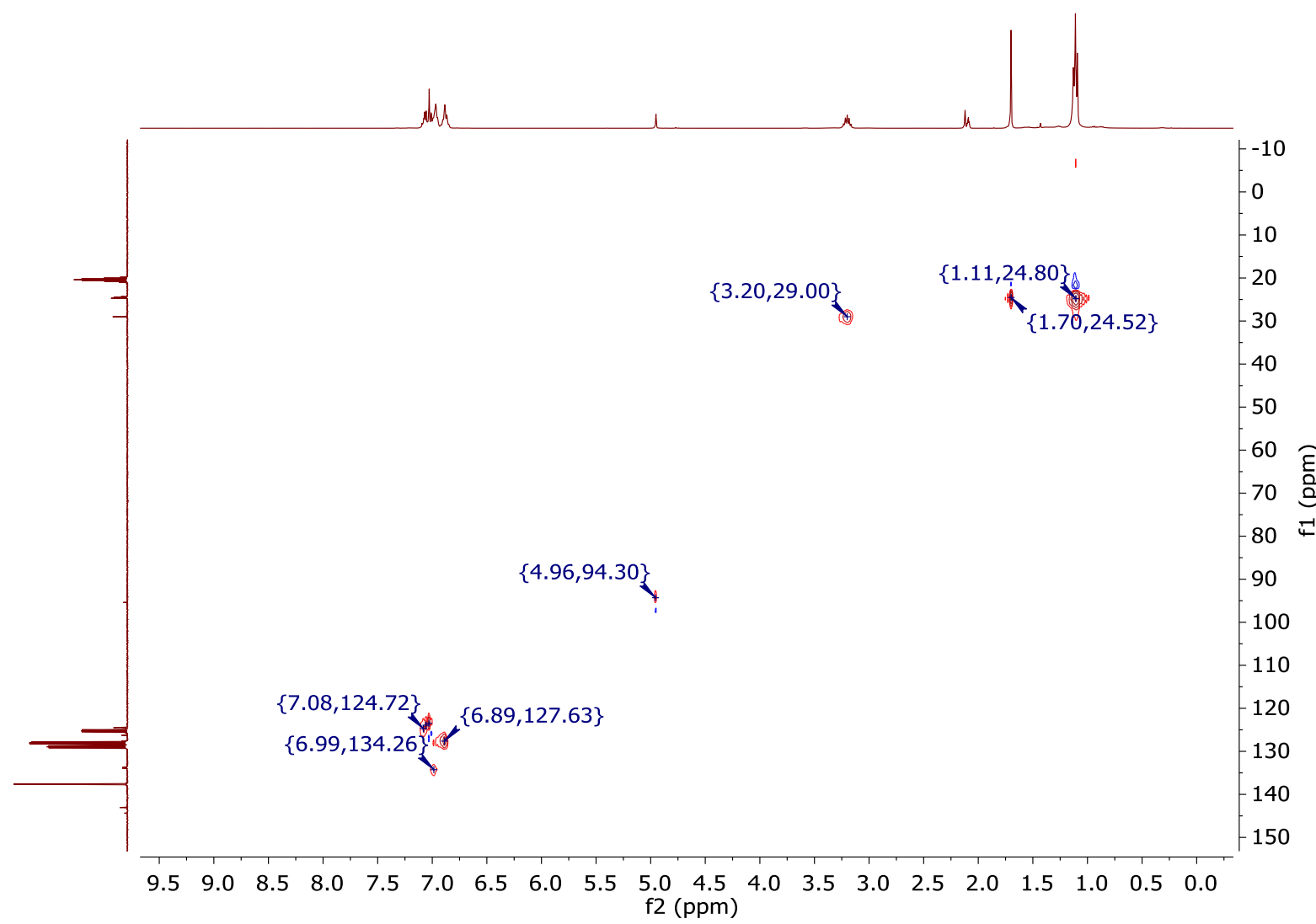

Figure S6: ${ }^{31} \mathrm{C}-{ }^{1} \mathrm{H}$ HSQC NMR spectrum (349 K, d8-toluene) of compound $6 \mathbf{a}$.

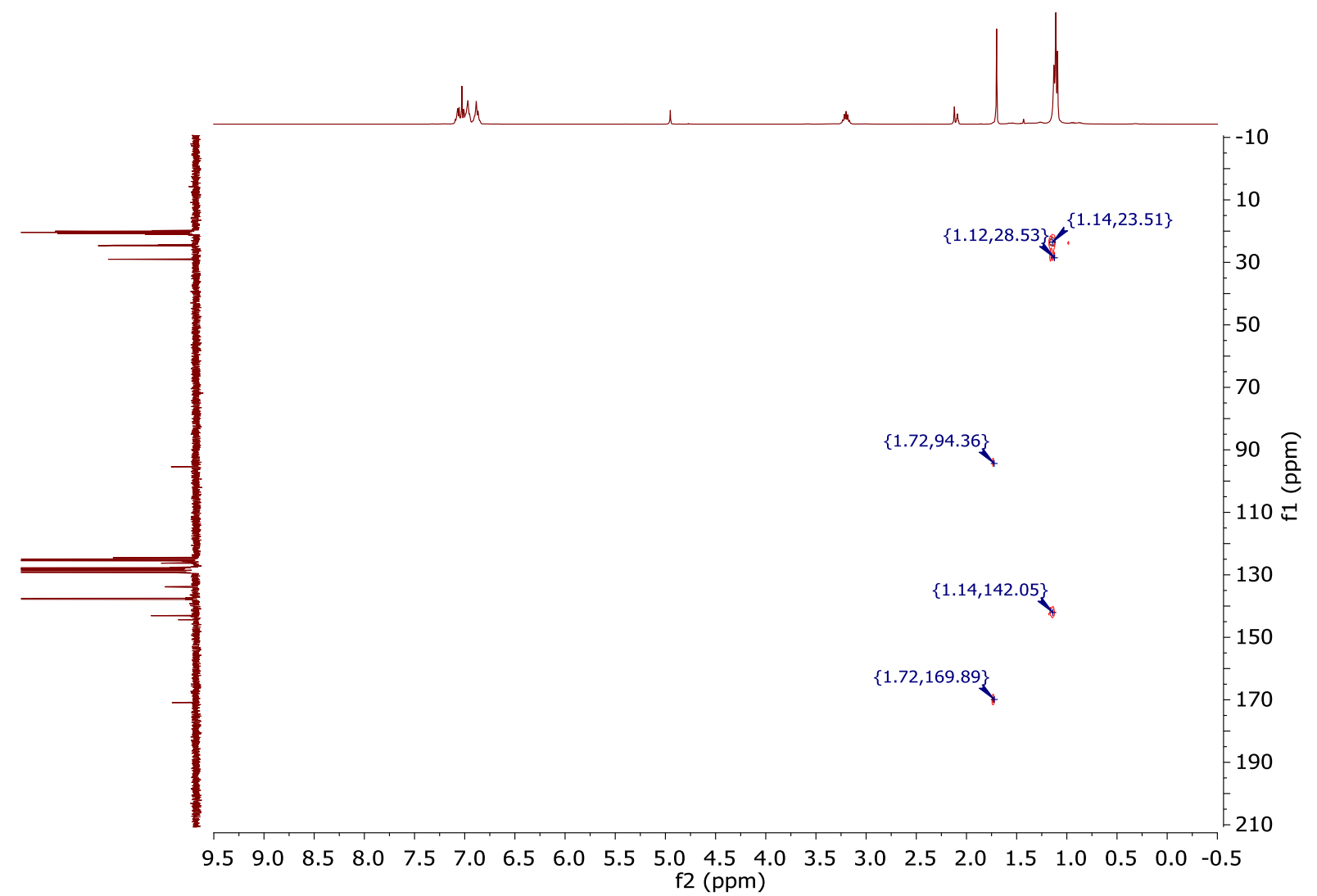

Figure S7: ${ }^{31} \mathrm{C}-{ }^{1} \mathrm{H}$ HMBC NMR spectrum (349 K, d8-toluene) of compound 6a. 


\section{Compound 6b}

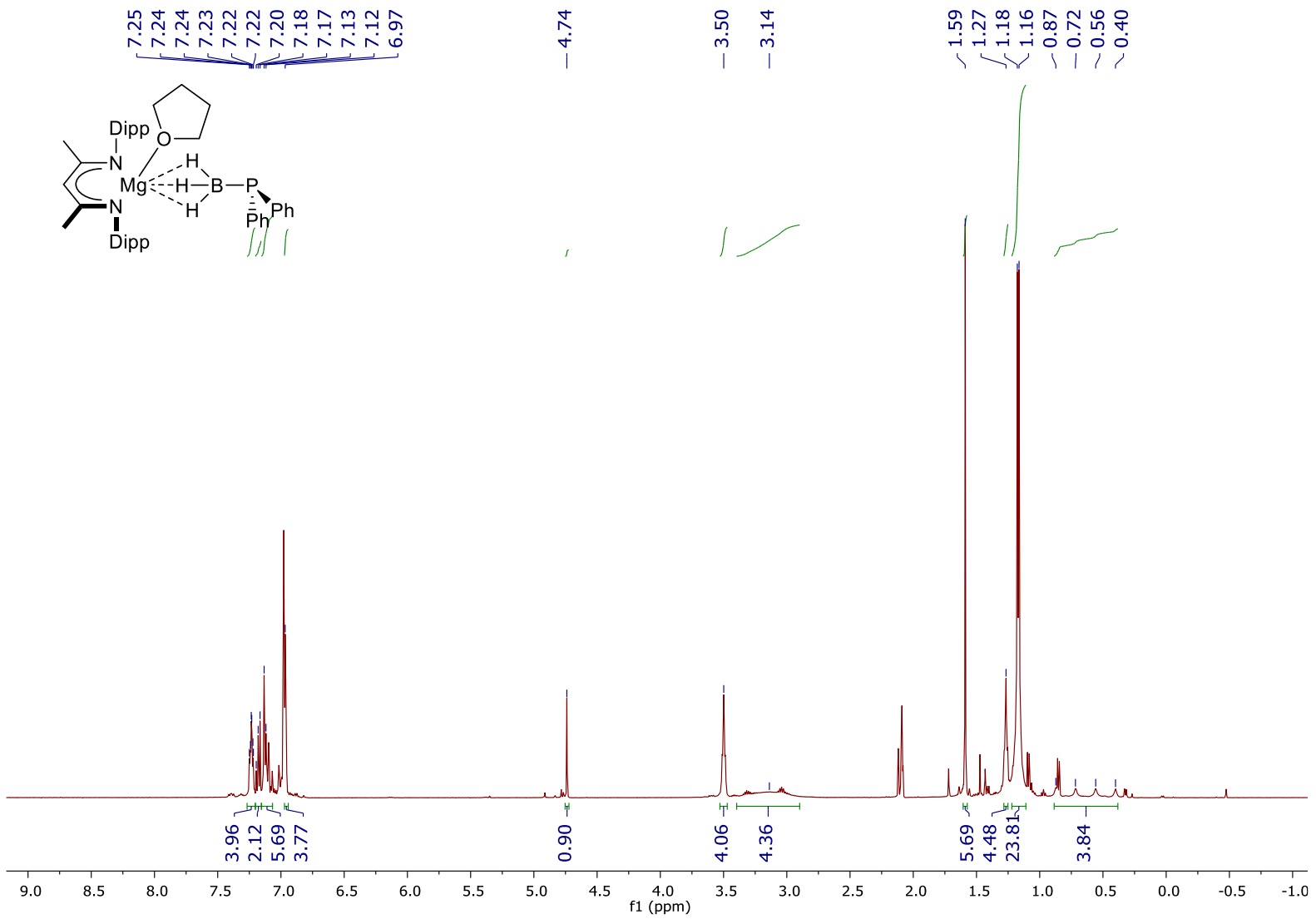

Figure S8: ${ }^{1} \mathrm{H}$ NMR spectrum ( $500 \mathrm{MHz}, 298 \mathrm{~K}$, d8-toluene) of compound $6 \mathbf{b}$.

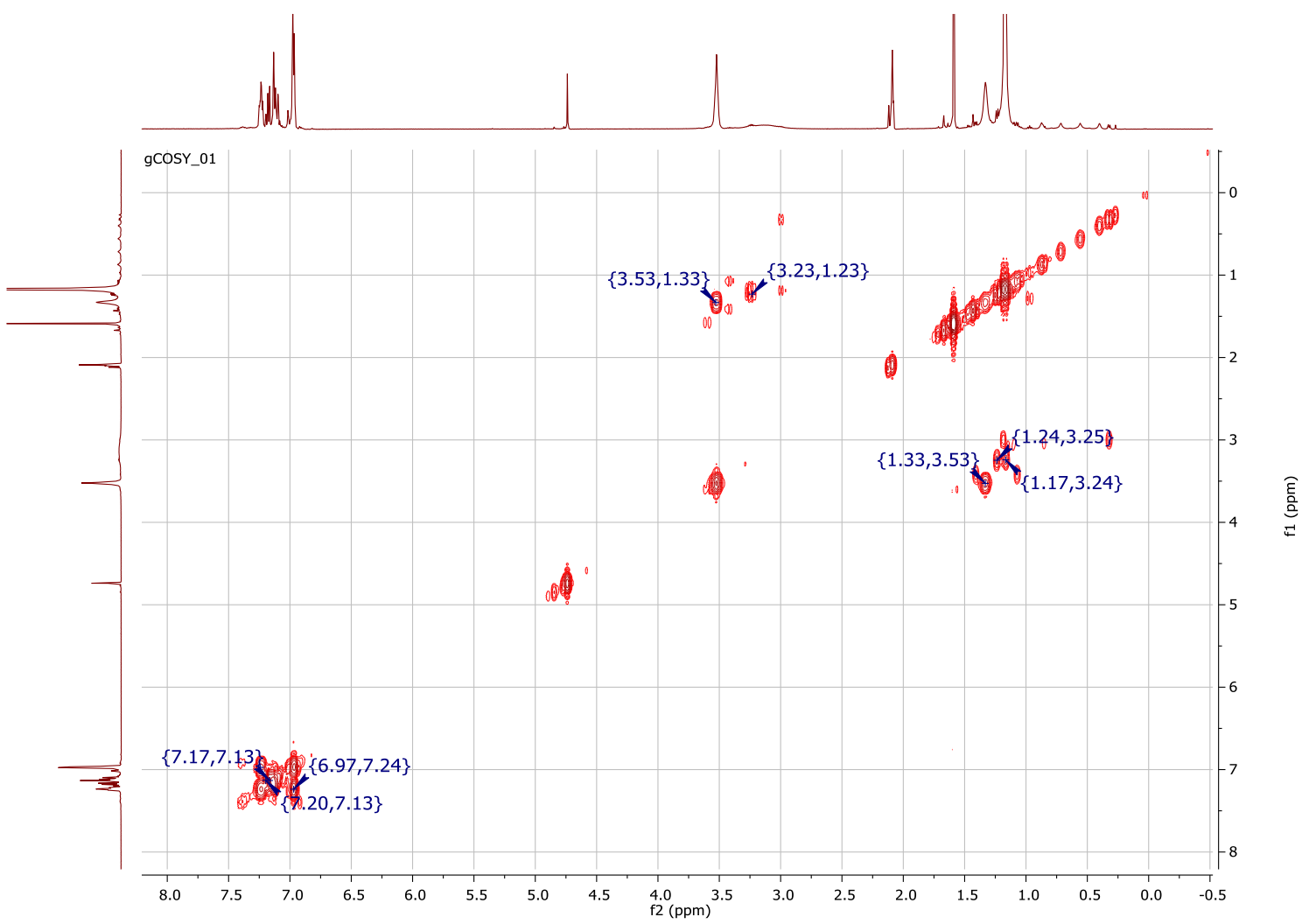

Figure S9: ${ }^{1} \mathrm{H}-{ }^{1} \mathrm{H}$ COSY NMR spectrum (298 K, d8-toluene) of compound $\mathbf{6 b}$. 


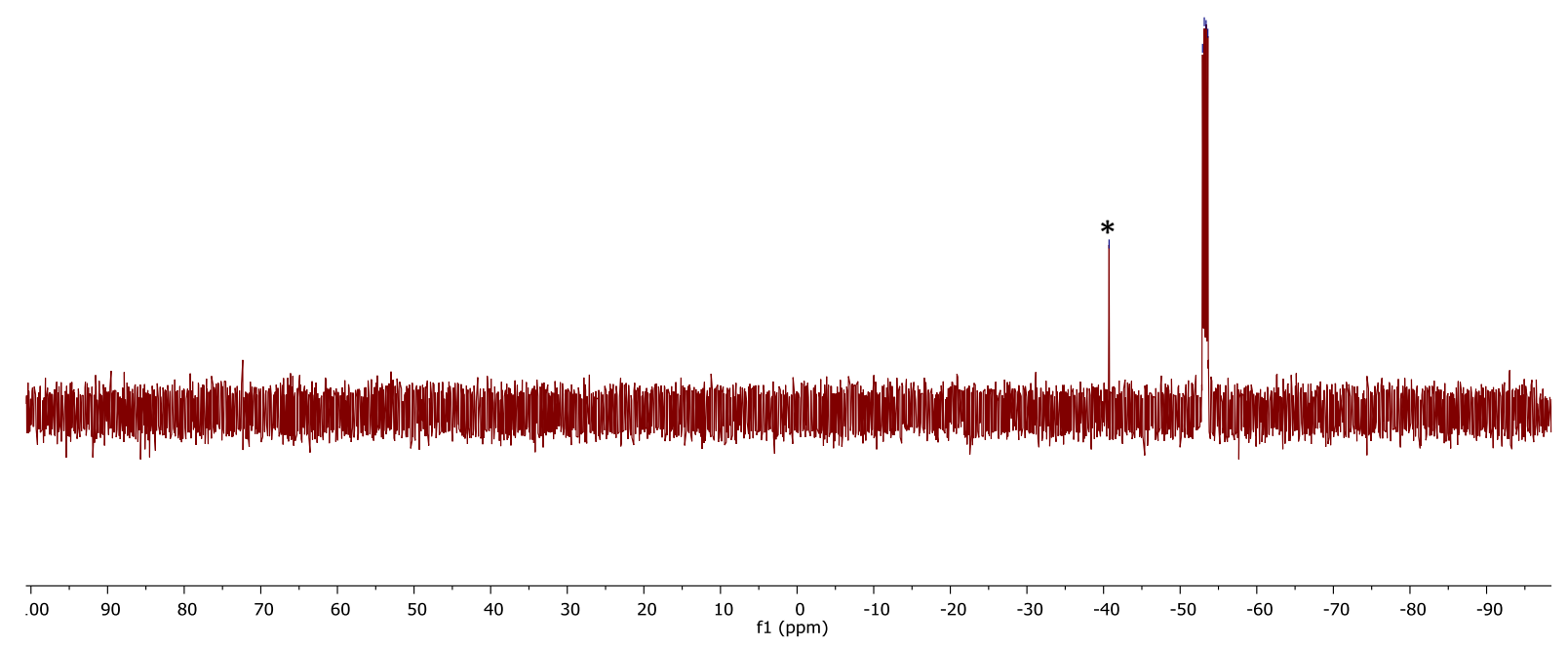

Figure S10: ${ }^{31} \mathrm{P}\left\{{ }^{1} \mathrm{H}\right\}$ NMR spectrum (202 MHz, $298 \mathrm{~K}$, d8-toluene) of compound $6 \mathbf{6 b} .{ }^{*}$ trace diphenylphosphine impurity.
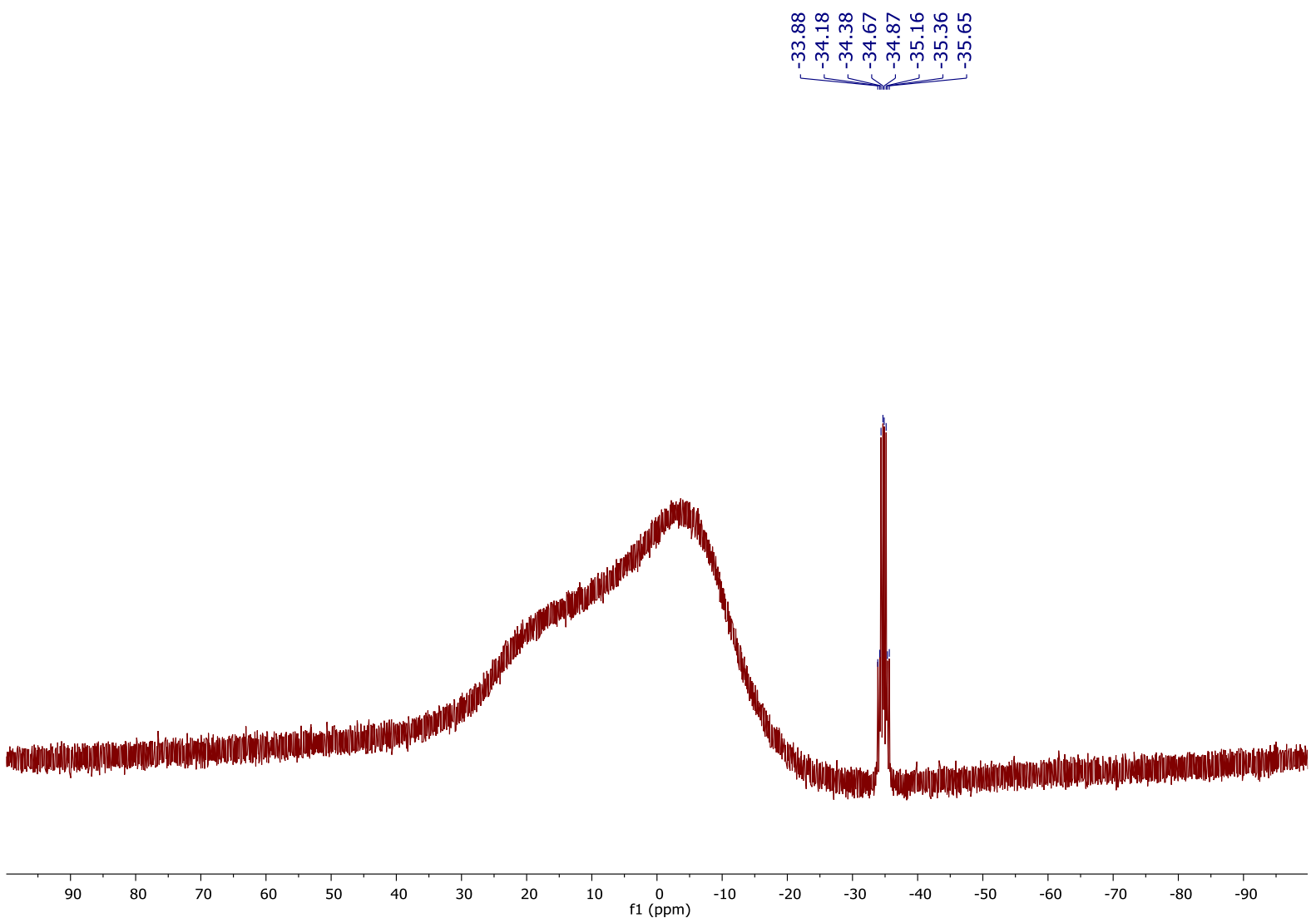

Figure S11: ${ }^{11} \mathrm{~B}$ NMR spectrum (160 MHz, $298 \mathrm{~K}$, d8-toluene) of compound $6 \mathbf{b}$. 


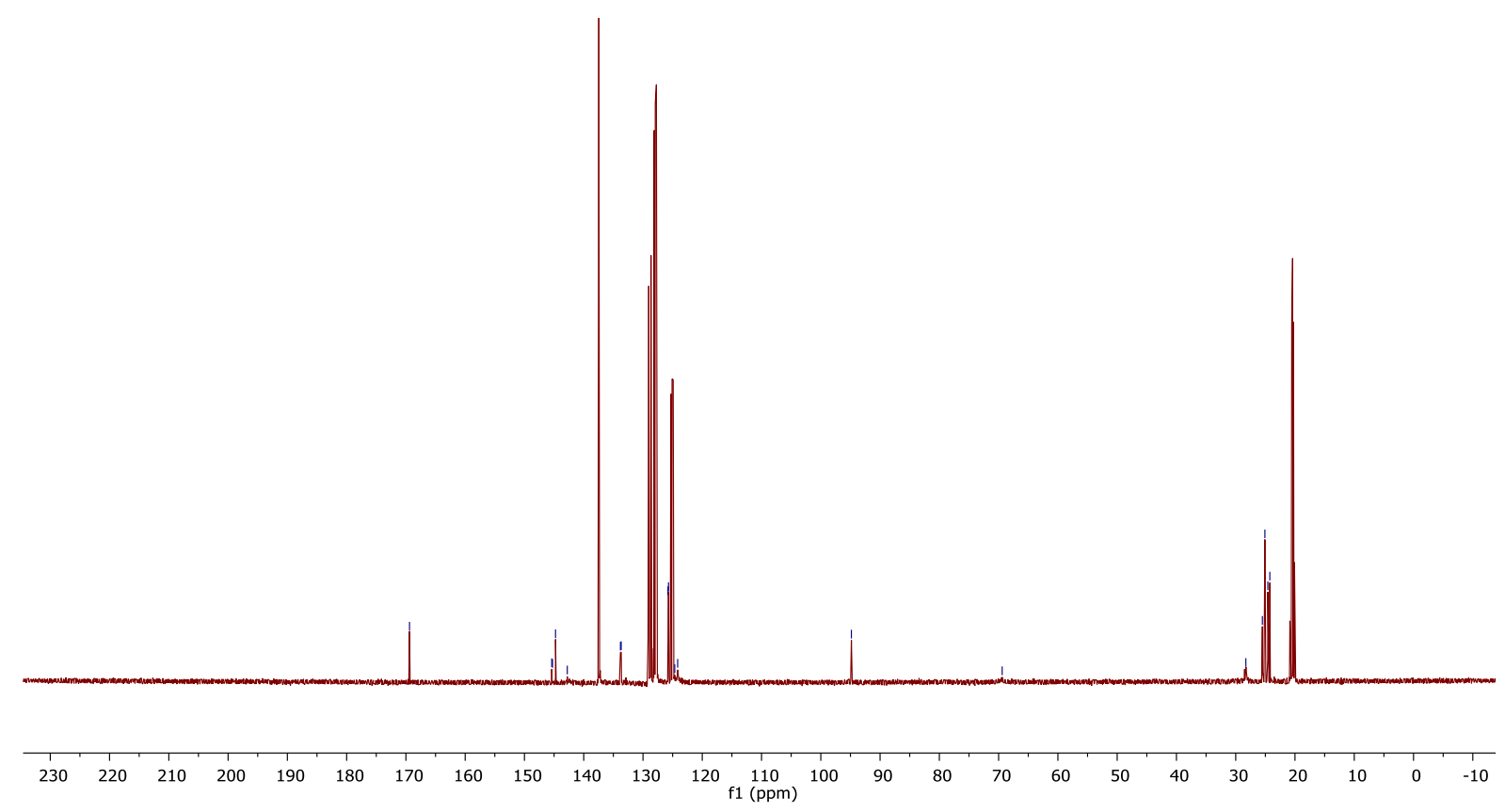

Figure $\mathrm{S} 12:{ }^{31} \mathrm{C}\left\{{ }^{1} \mathrm{H}\right\}$ NMR spectrum (126 MHz, $298 \mathrm{~K}$, d $\mathrm{d}_{8}$-toluene) of compound $\mathbf{6 b}$.

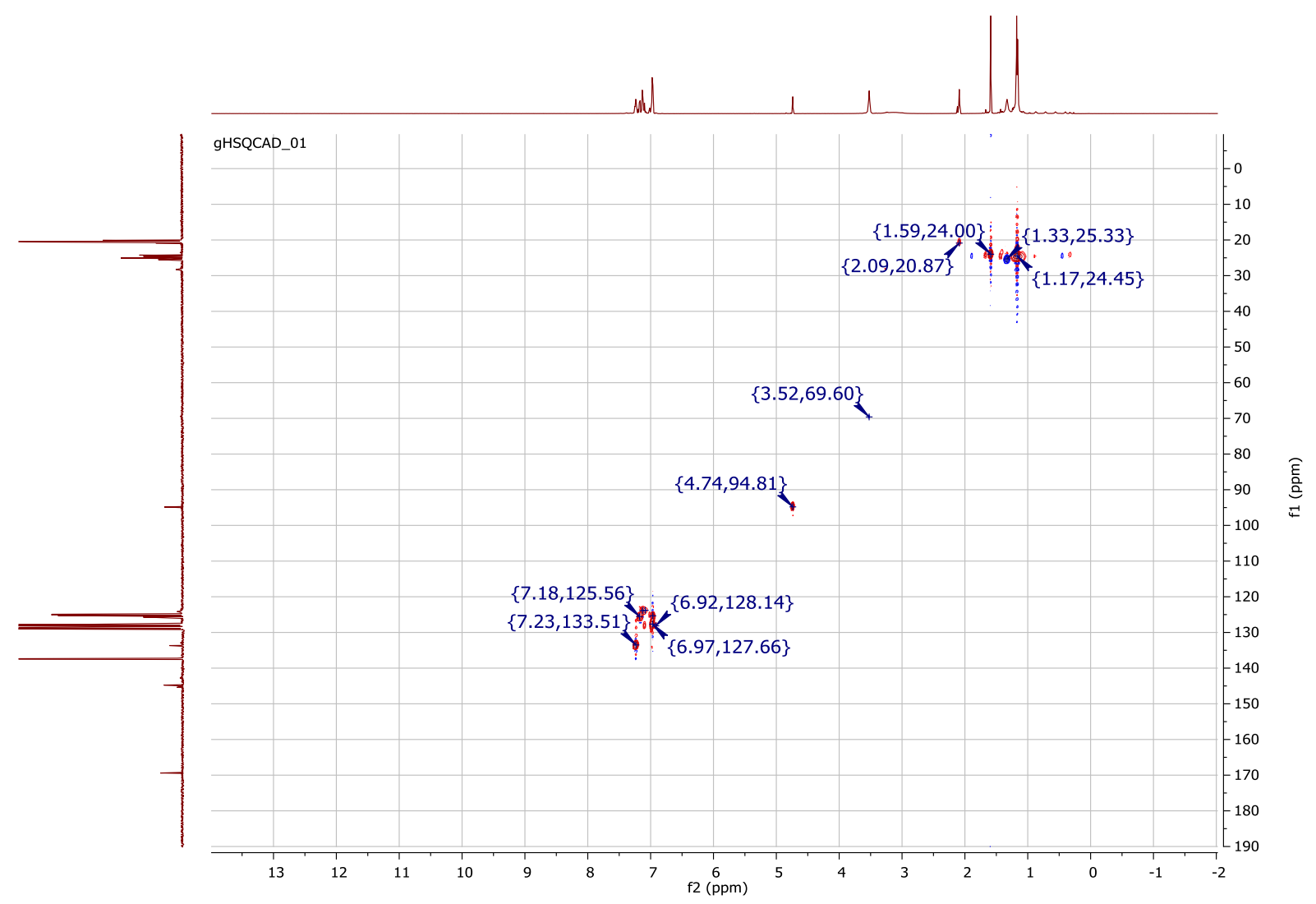

Figure S13: ${ }^{13} \mathrm{C}-{ }^{1} \mathrm{H}$ HSQC NMR spectrum (298 K, d $\mathrm{d}_{8}$-toluene) of compound $\mathbf{6 b}$. 


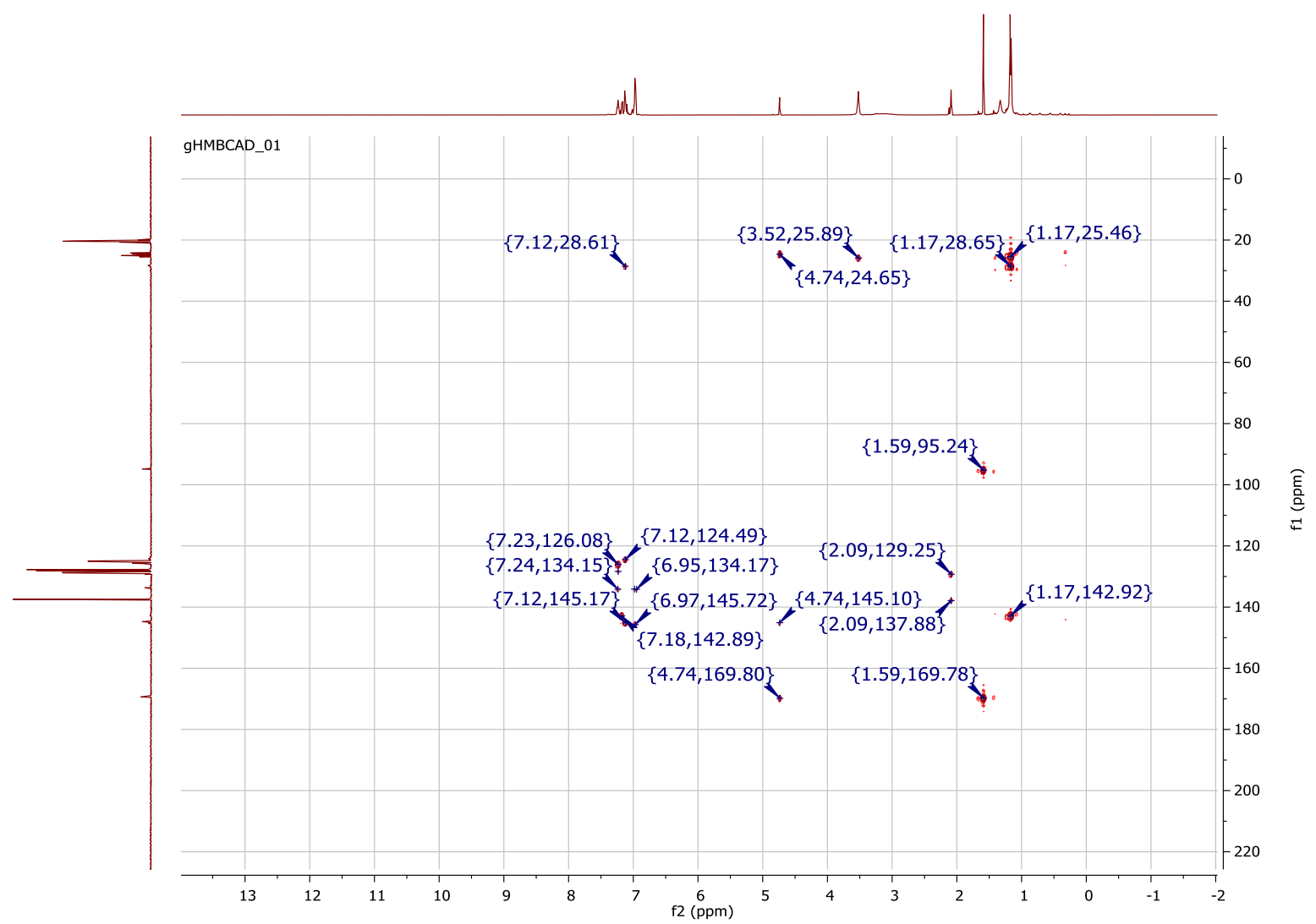

Figure S14: ${ }^{13} \mathrm{C}-{ }^{1} \mathrm{H}$ HMBC NMR spectrum (298 K, d 8 -toluene) of compound $6 \mathbf{b}$.

\section{Compound 7a}

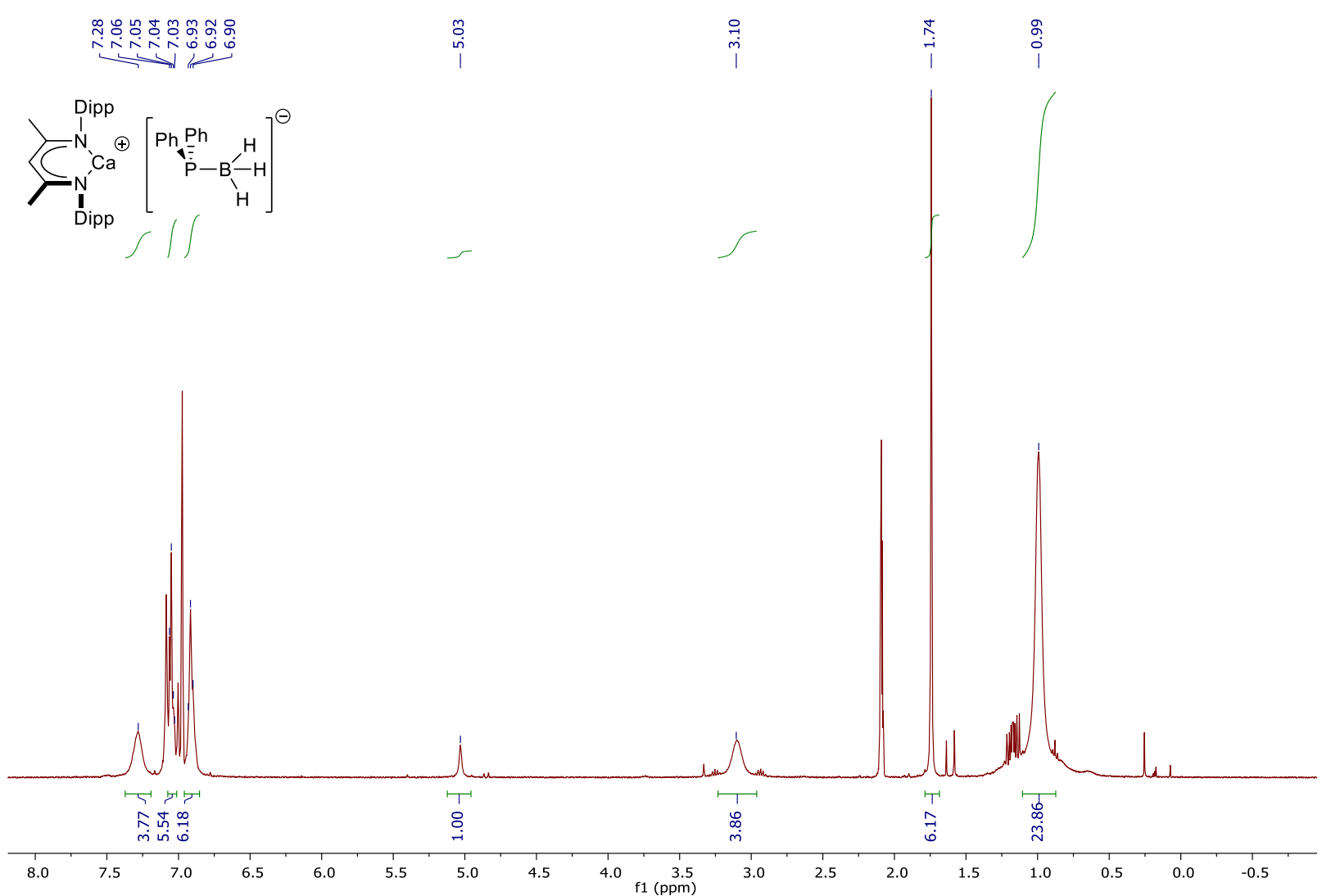

Figure S15: ${ }^{1} \mathrm{H}$ NMR spectrum (400 MHz, $320.6 \mathrm{~K}$, d8-toluene) of compound 7a. 


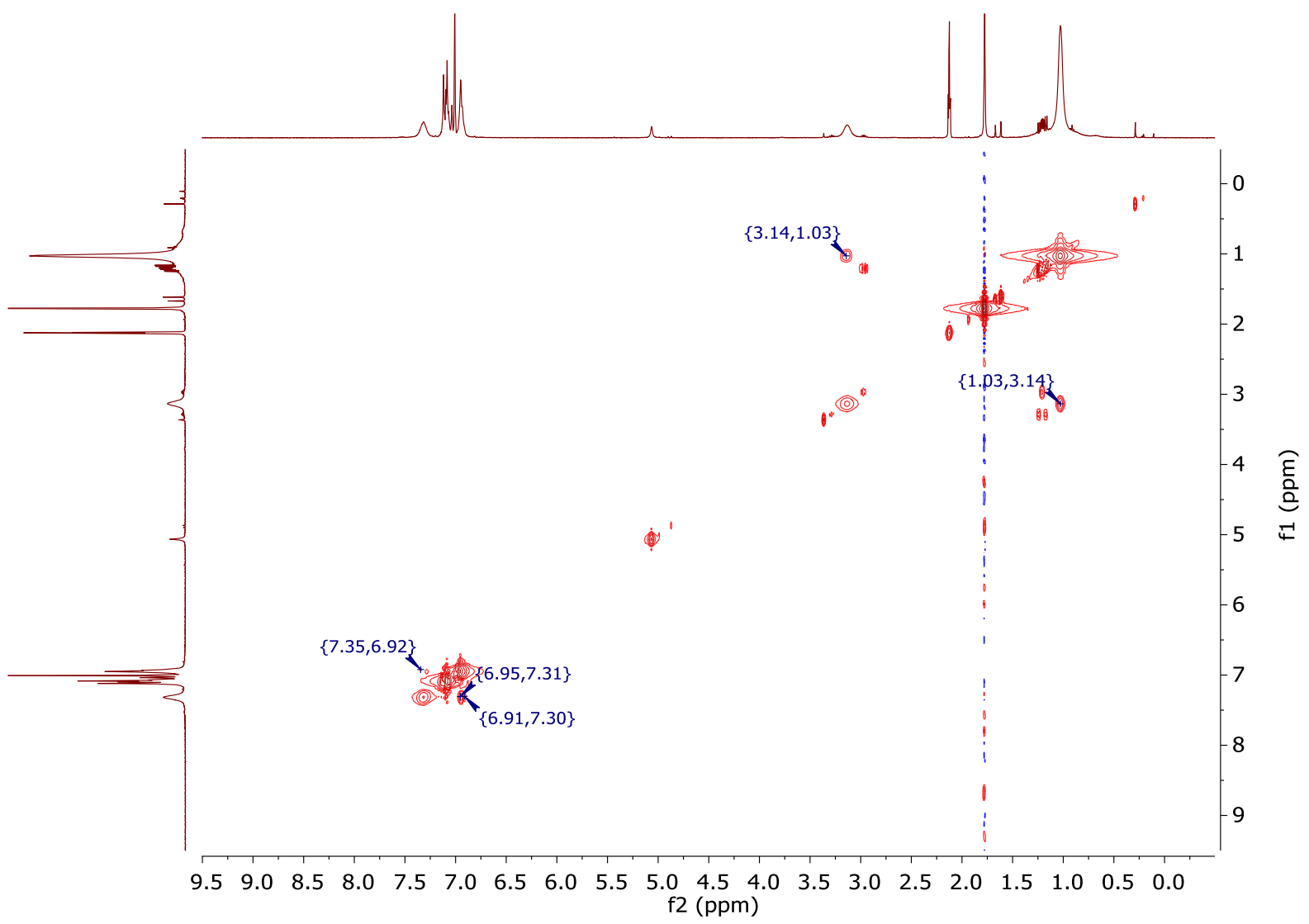

Figure S16: ${ }^{1} \mathrm{H}-{ }^{1} \mathrm{H}$ COSY NMR spectrum (320.6 $\mathrm{K}$ d8-toluene) of compound $7 \mathrm{a}$.

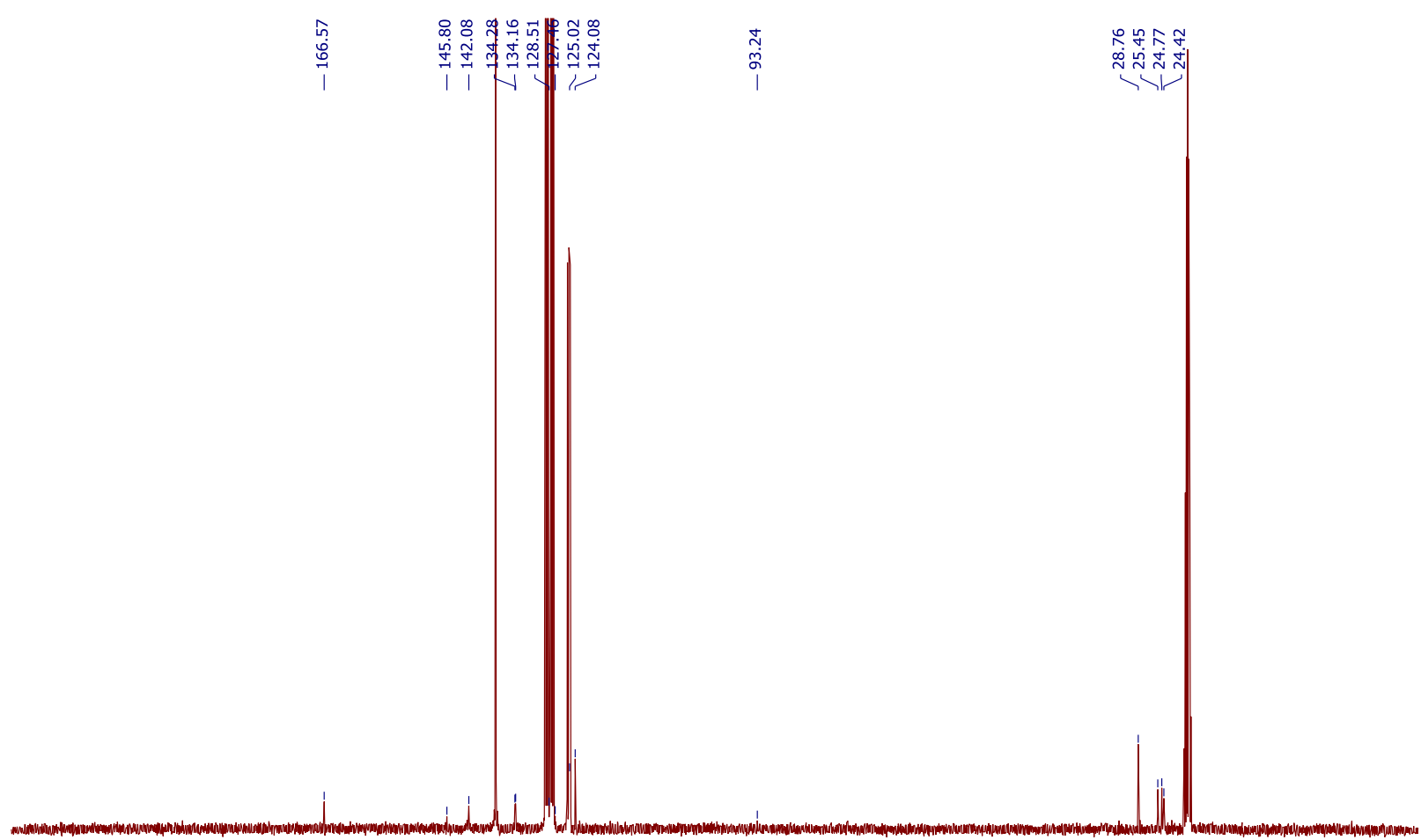

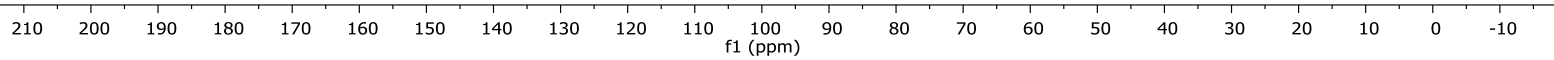

Figure S17: ${ }^{31} \mathrm{C}\left\{{ }^{1} \mathrm{H}\right\}$ NMR spectrum (101 MHz, $320.6 \mathrm{~K}$, d $\mathrm{d}_{8}$-toluene) of compound 7a. 


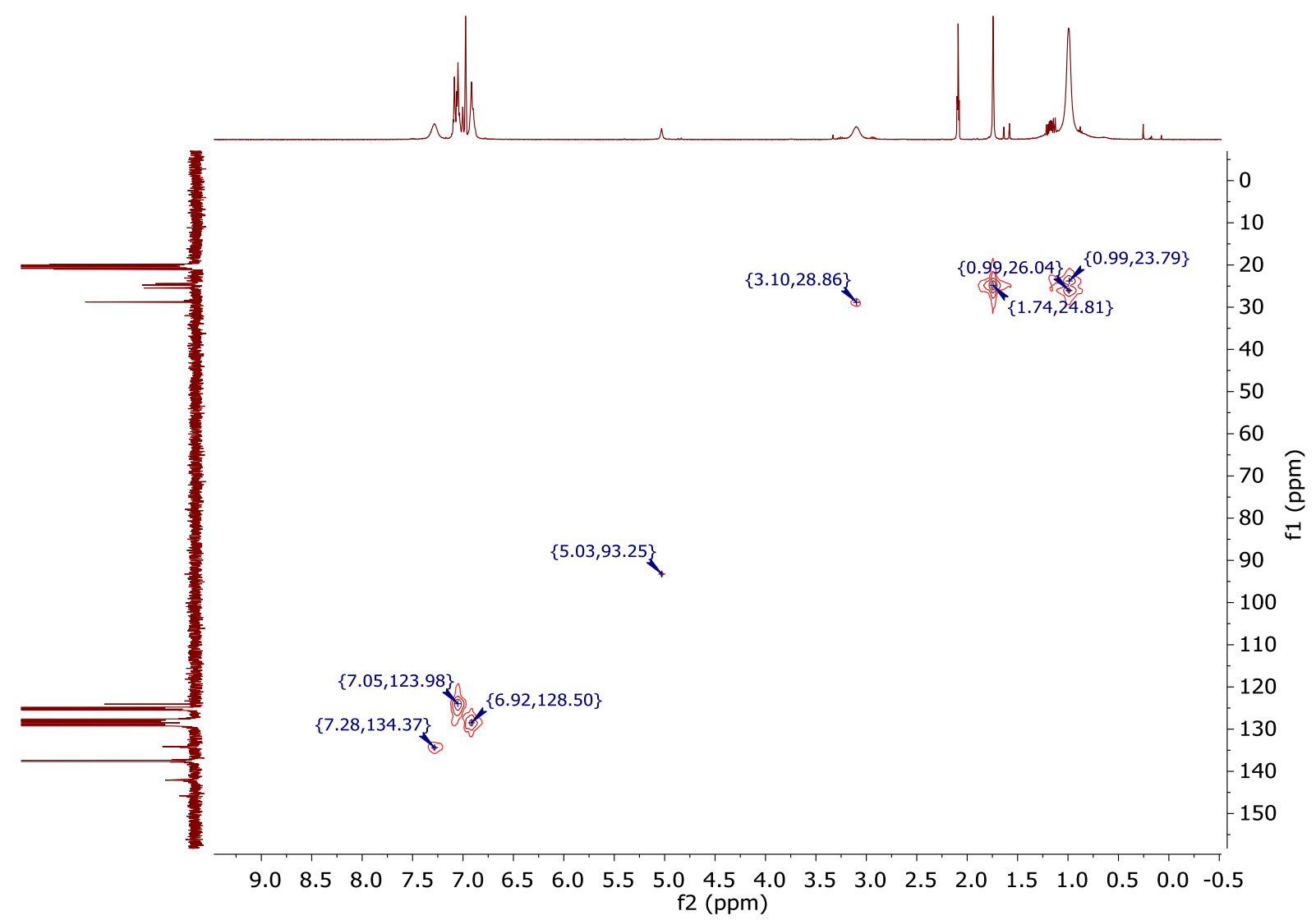

Figure S18: ${ }^{31} \mathrm{C}-{ }^{1} \mathrm{H}$ HSQC NMR spectrum (320.6 K, $\mathrm{d}_{8}$-toluene) of compound $7 \mathrm{a}$.

$$
\stackrel{\substack{p \\ \dot{p}}}{i}
$$

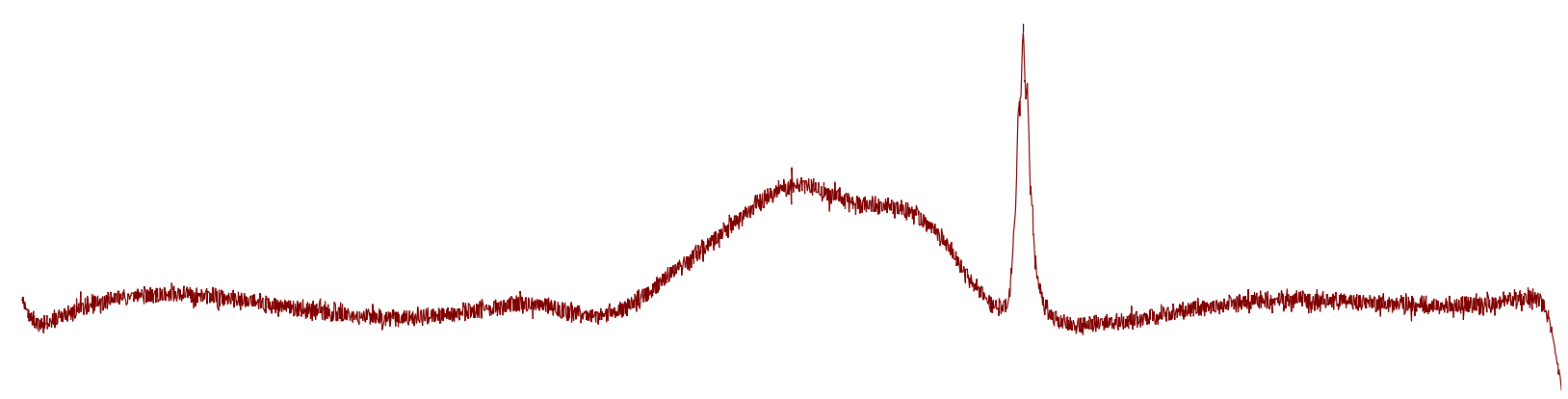

$\begin{array}{llllllllll}90 & 80 & 70 & 60 & 50 & 40 & 30 & 20 & 10 & \begin{array}{c}0 \\ \mathrm{f} 1(\mathrm{ppm})\end{array} \\ & & & & & & & \end{array}$

Figure S19: ${ }^{11} \mathrm{~B}$ NMR spectrum (128 MHz, $320.6 \mathrm{~K}$, $\mathrm{d}_{8}$-toluene) of compound 7a. 

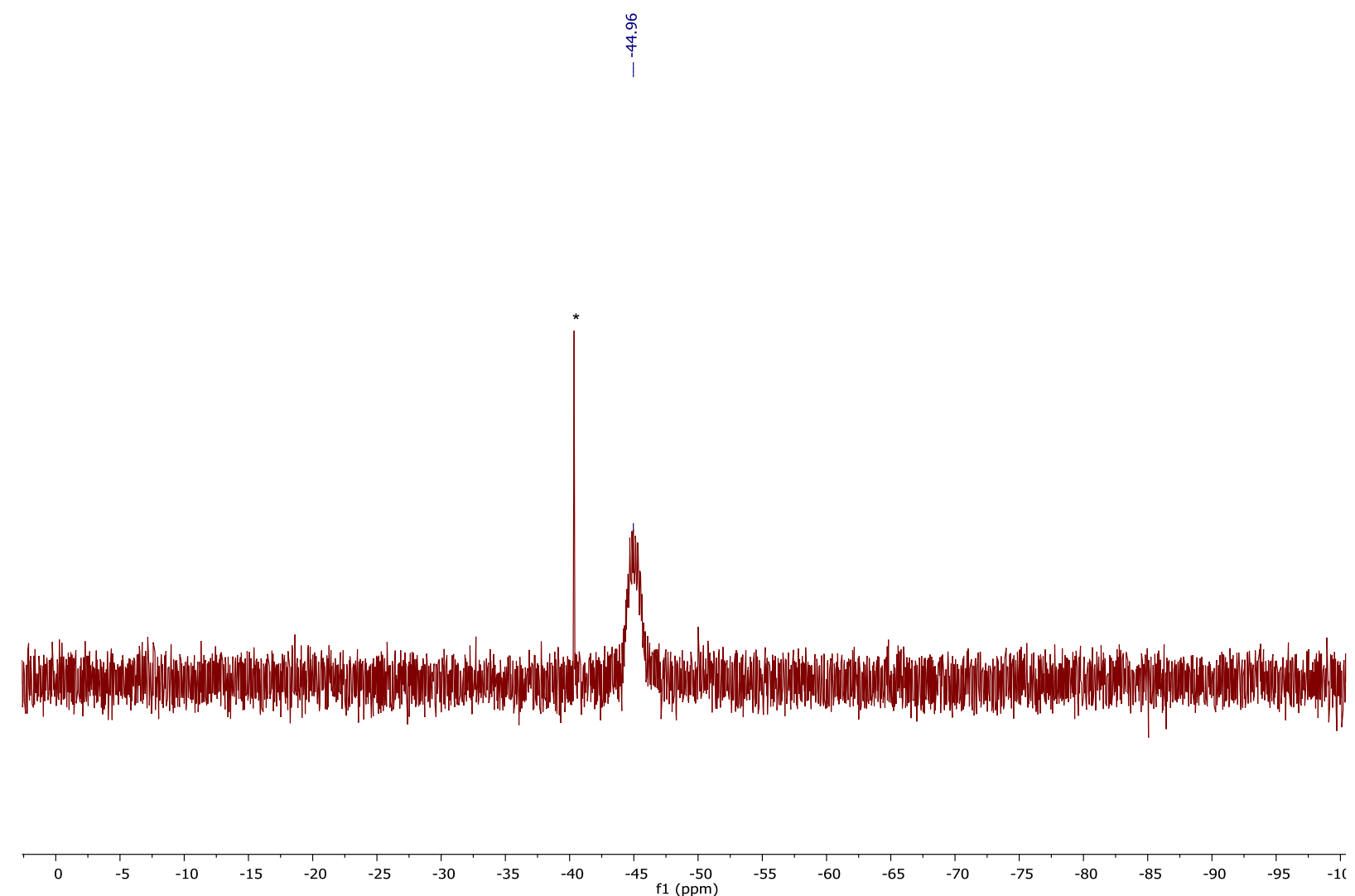

Figure S20: ${ }^{31} \mathrm{P}\left\{{ }^{1} \mathrm{H}\right\}$ NMR spectrum (162 MHz, 320.6 K d8-toluene) of compound 7a. * trace diphenylphosphine impurity.
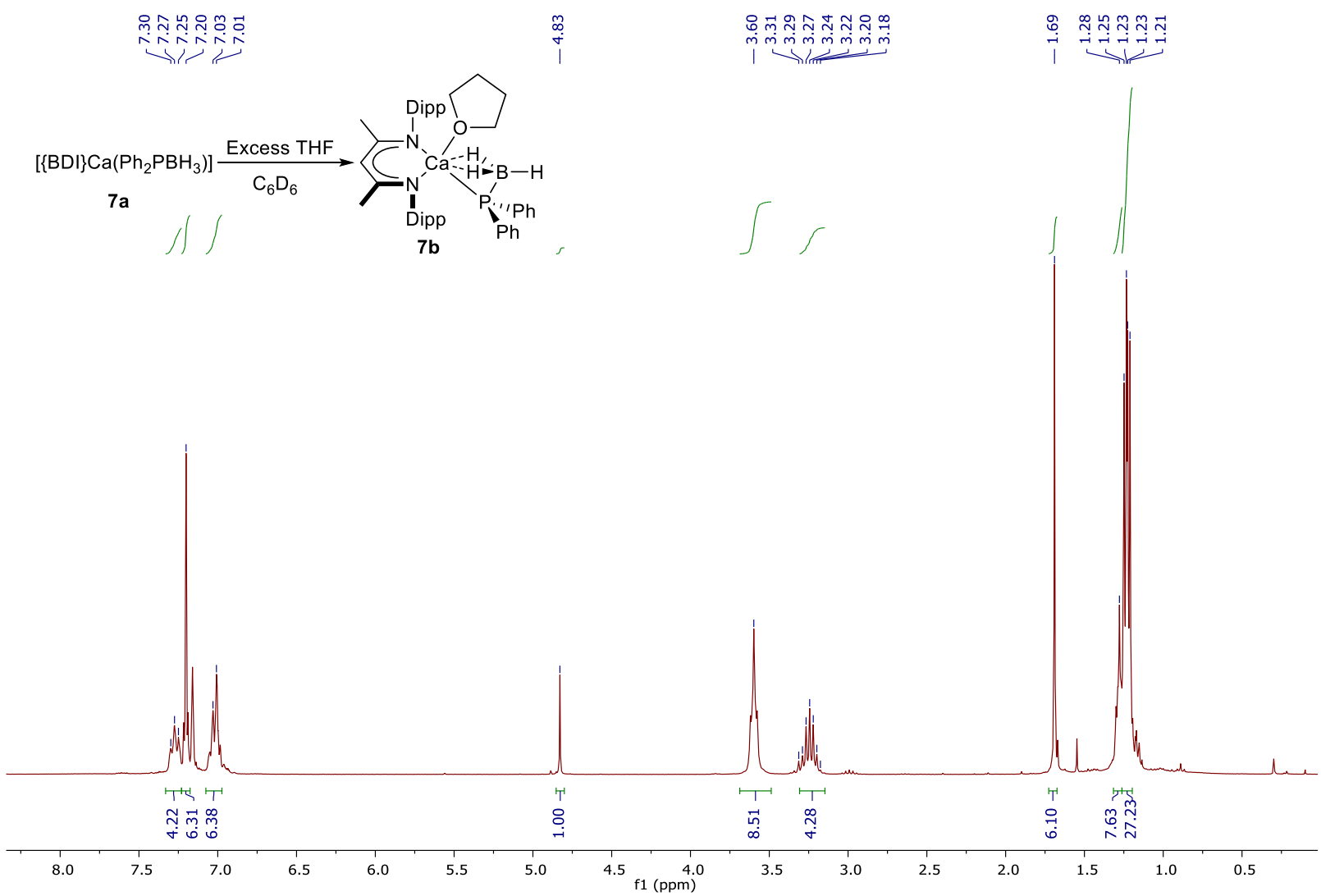

Figure S21: ${ }^{1} \mathrm{H}$ NMR spectrum $\left(300 \mathrm{MHz}, 298 \mathrm{~K}, \mathrm{C}_{6} \mathrm{D}_{6}\right)$ resulting from the addition of two equivalents of THF to a $\mathrm{C}_{6} \mathrm{D}_{6}$ solution of $7 \mathbf{a}$. 


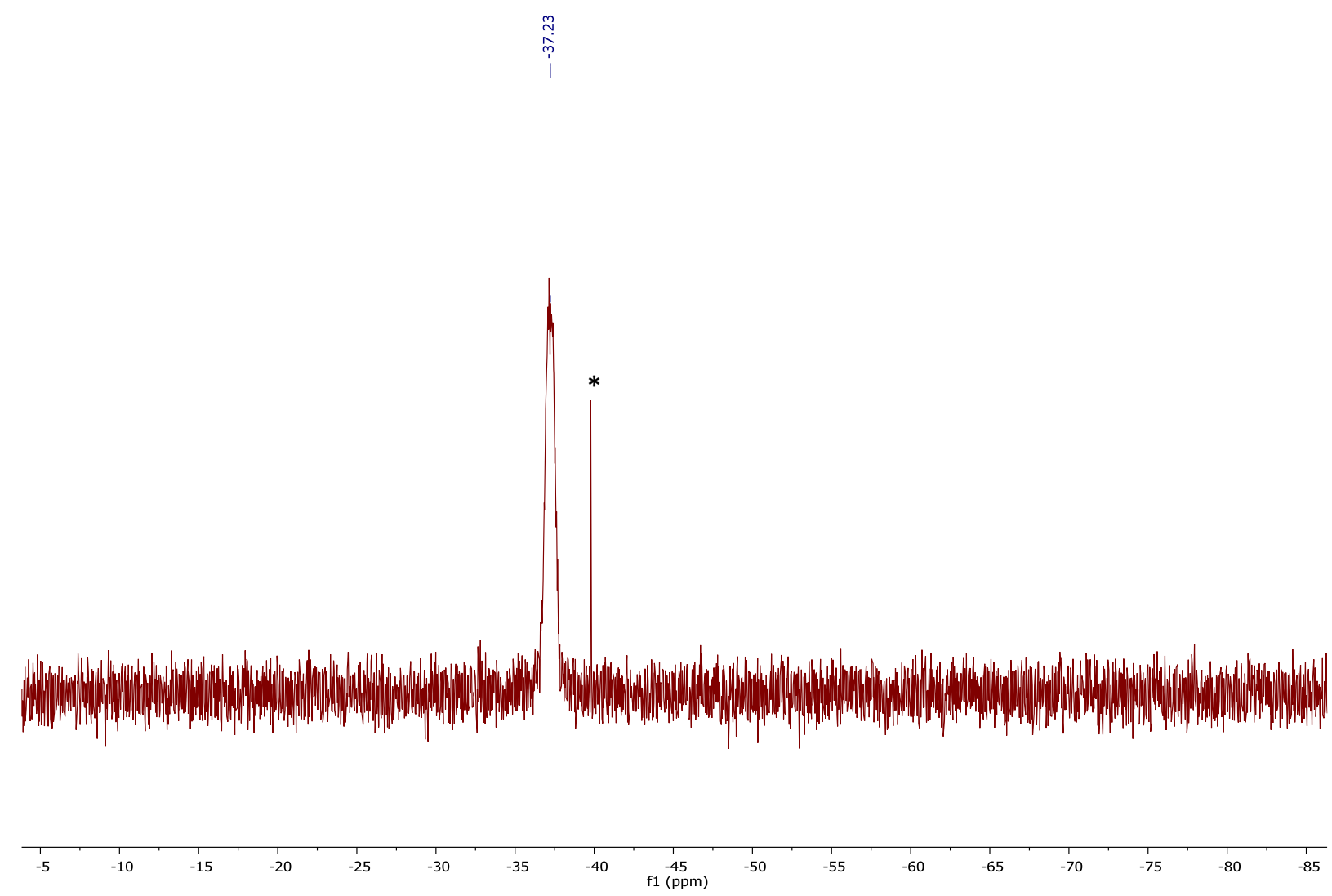

Figure S22: ${ }^{31} \mathrm{P}\left\{{ }^{1} \mathrm{H}\right\}$ NMR spectrum $\left(122 \mathrm{MHz}, 298 \mathrm{~K}, \mathrm{C}_{6} \mathrm{D}_{6}\right)$ resulting from the addition of two equivalents of THF to $\mathrm{a}_{6} \mathrm{D}_{6}$ solution of $7 \mathrm{a} .{ }^{*}$ trace diphenylphosphine impurity.

๙ָ

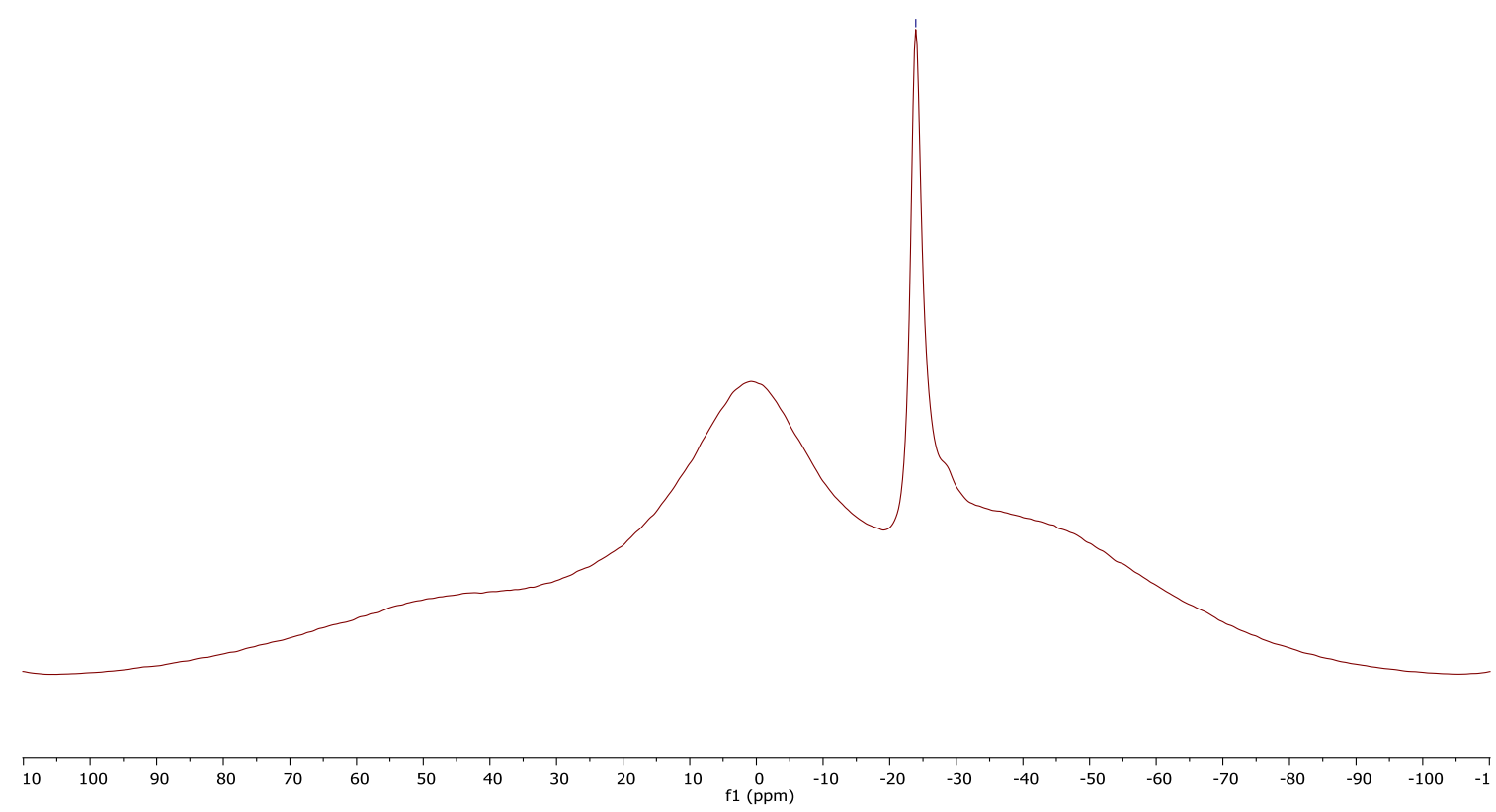

Figure S23: ${ }^{11} \mathrm{~B}$ NMR spectrum $\left(96 \mathrm{MHz}, 298 \mathrm{~K}, \mathrm{C}_{6} \mathrm{D}_{6}\right)$ resulting from the addition of two equivalents of THF to a $\mathrm{C}_{6} \mathrm{D}_{6}$ solution of $7 \mathrm{a}$.

\section{Compound $7 b$}




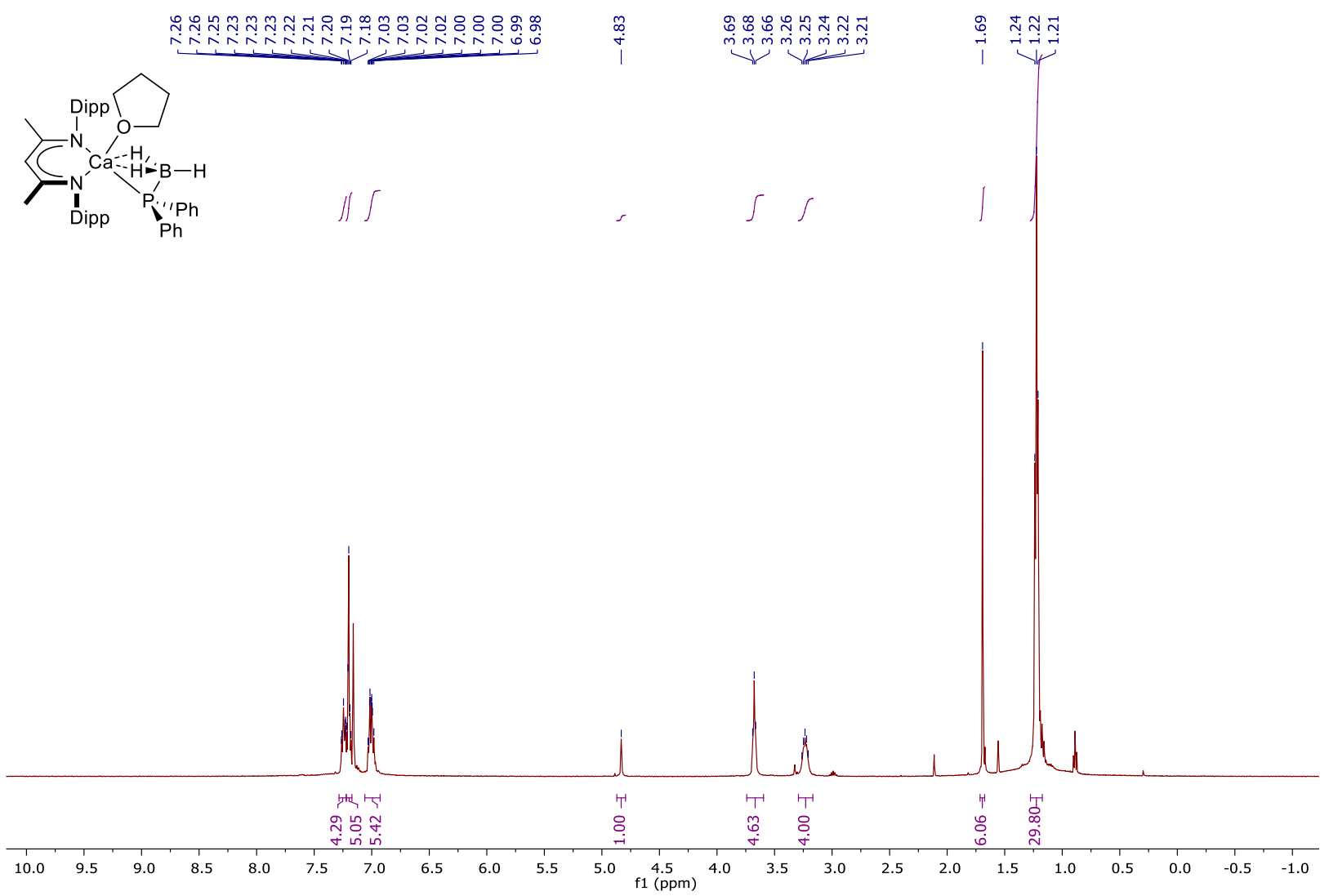

Figure S24: ${ }^{1} \mathrm{H}$ NMR spectrum $\left(500 \mathrm{MHz}, 298 \mathrm{~K}, \mathrm{C}_{6} \mathrm{D}_{6}\right)$ of compound $7 \mathbf{b}$.

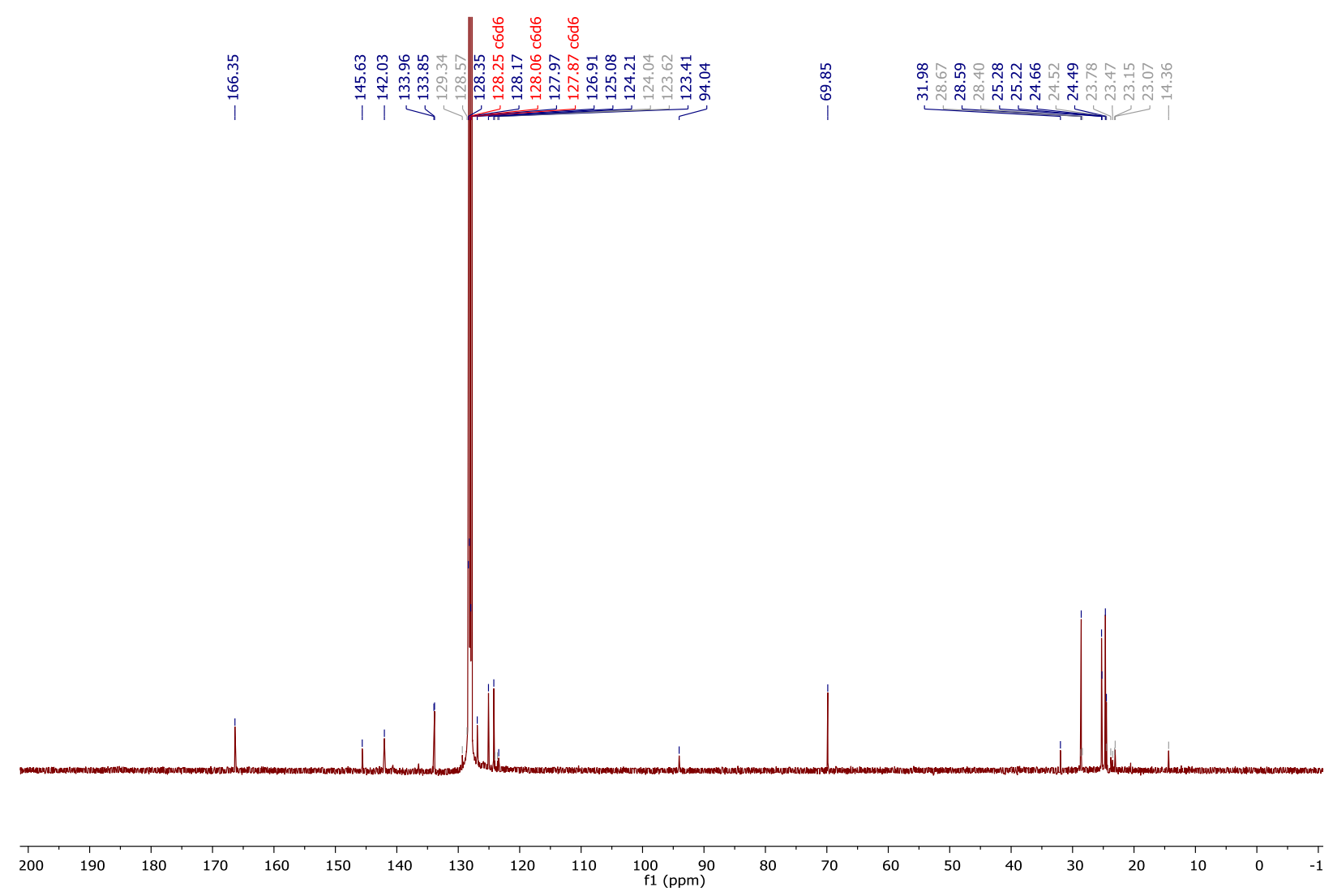

Figure S25: ${ }^{31} \mathrm{C}\left\{{ }^{1} \mathrm{H}\right\}$ NMR spectrum (126 MHz, $\left.298 \mathrm{~K}, \mathrm{C}_{6} \mathrm{D}_{6}\right)$ of compound $7 \mathbf{b}$. 


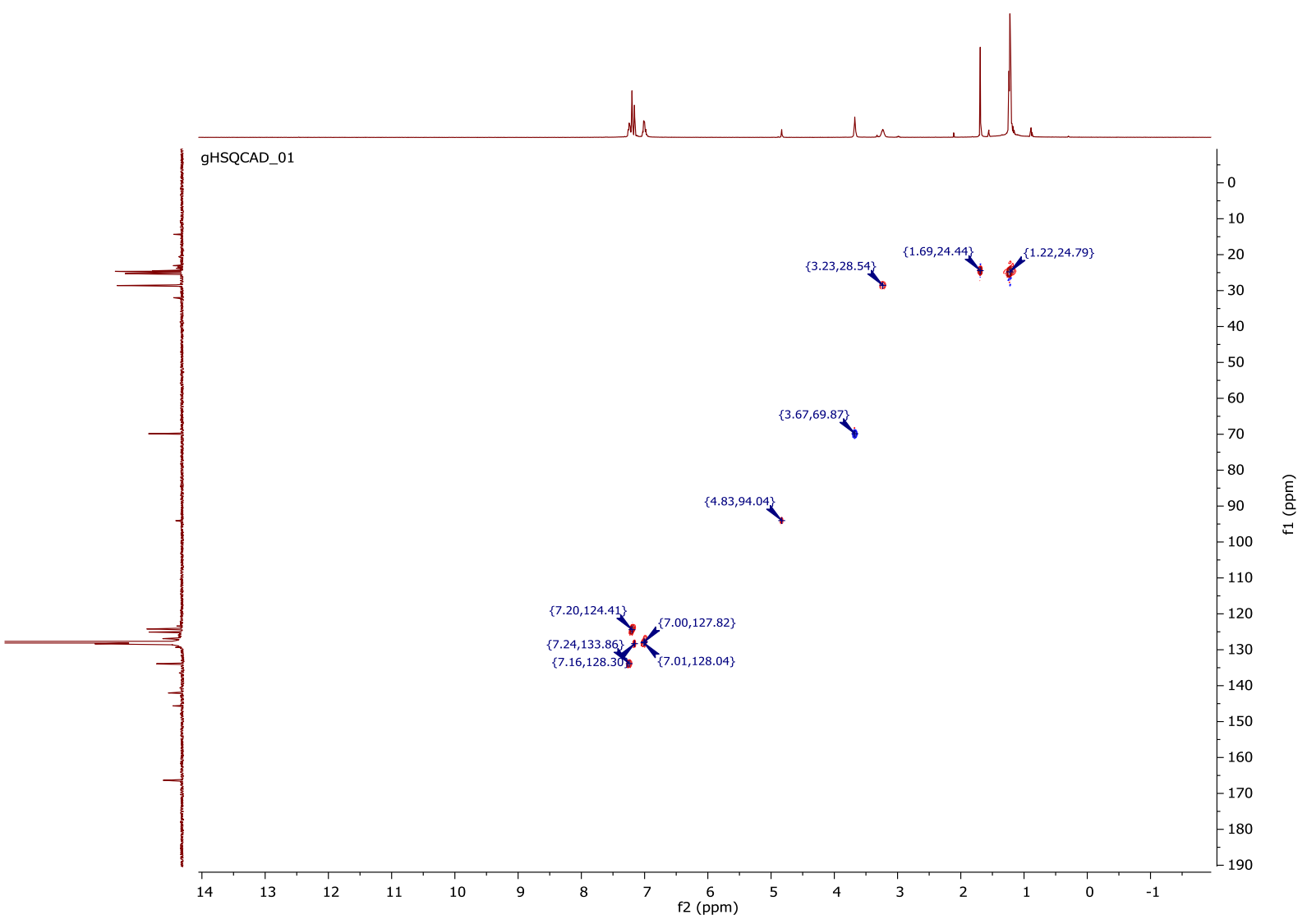

Figure S26: ${ }^{13} \mathrm{C}-{ }^{-1} \mathrm{H}$ HSQC NMR spectrum $\left(298 \mathrm{~K}, \mathrm{C}_{6} \mathrm{D}_{6}\right)$ of compound $7 \mathbf{b}$.

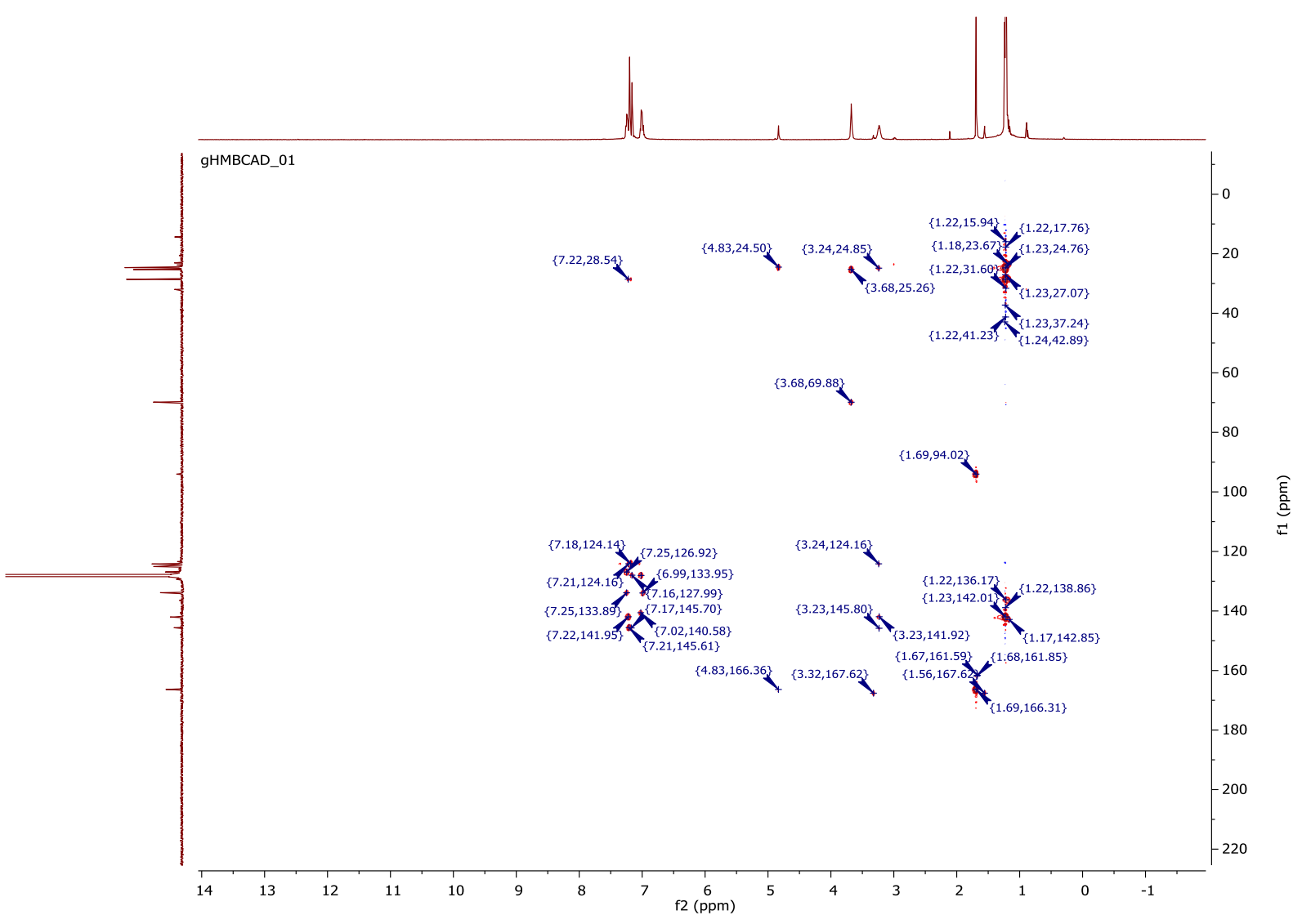

Figure S27: ${ }^{13} \mathrm{C}-{ }^{1} \mathrm{H}$ HMBC NMR spectrum $\left(298 \mathrm{~K}, \mathrm{C}_{6} \mathrm{D}_{6}\right)$ of compound $7 \mathbf{b}$. 


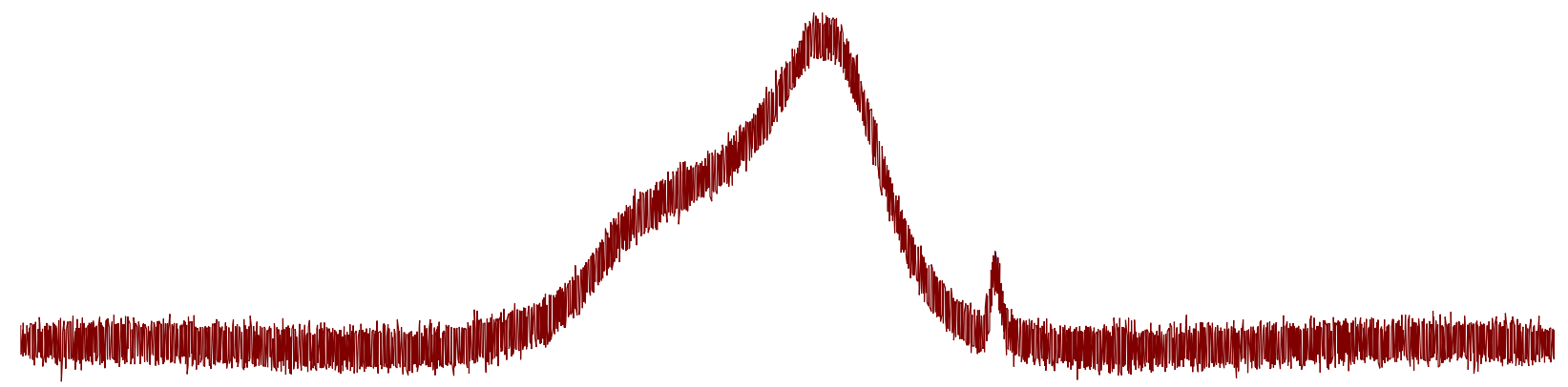

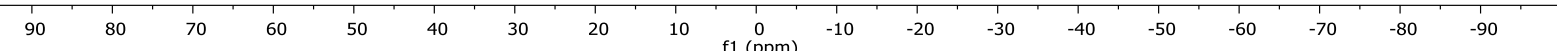

Figure S28: ${ }^{11} \mathrm{~B}$ NMR spectrum $\left(160 \mathrm{MHz}, 298 \mathrm{~K}, \mathrm{C}_{6} \mathrm{D}_{6}\right)$ of compound $7 \mathbf{b}$.

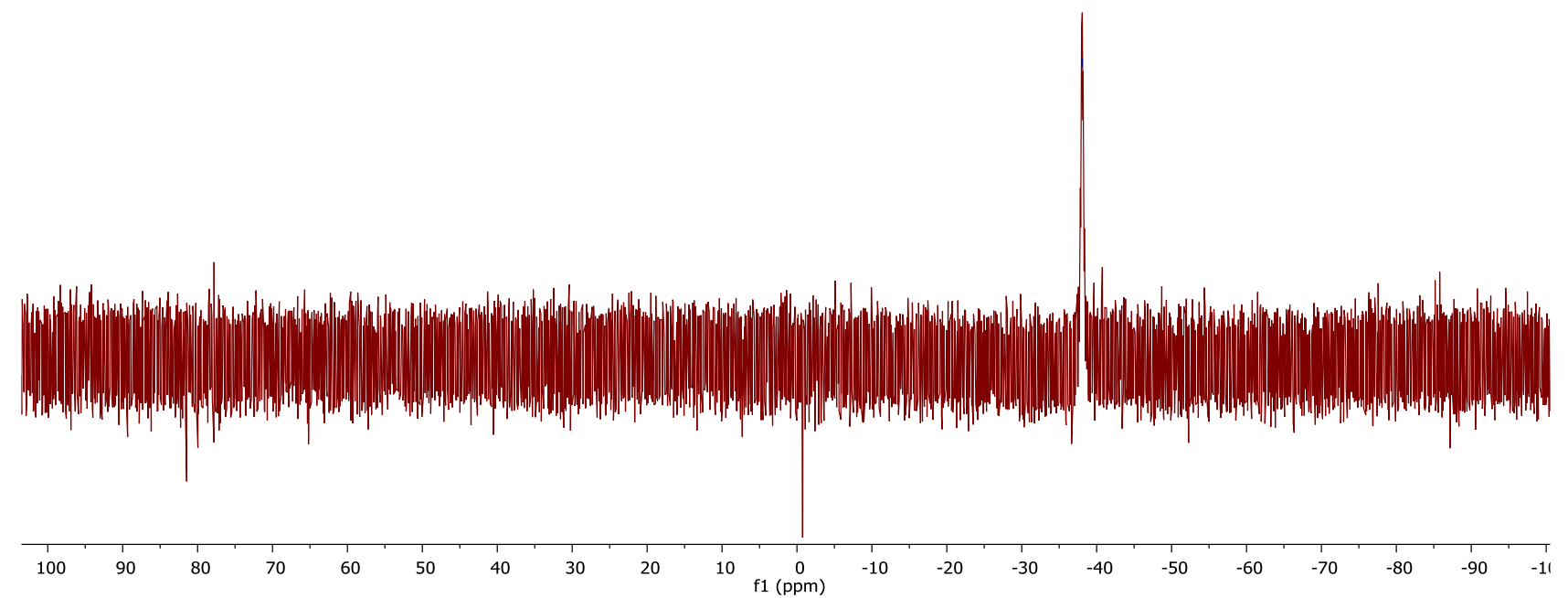


Figure S29: ${ }^{31} \mathrm{P}\left\{{ }^{1} \mathrm{H}\right\}$ NMR spectrum $\left(202 \mathrm{MHz}, 298 \mathrm{~K}, \mathrm{C}_{6} \mathrm{D}_{6}\right)$ of compound $\mathbf{7 b}$.

\section{Compound 8}

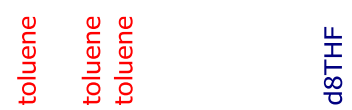

崖

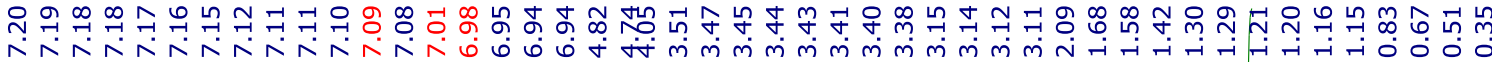

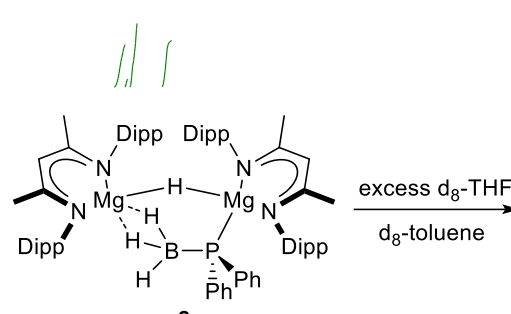

8

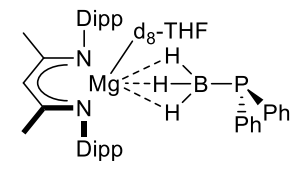

$6 b\left(d_{8}\right)$

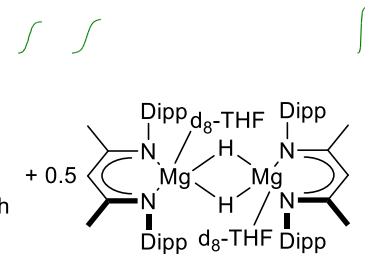

IIIa. $\left(d_{8}-T H F\right)_{2}$

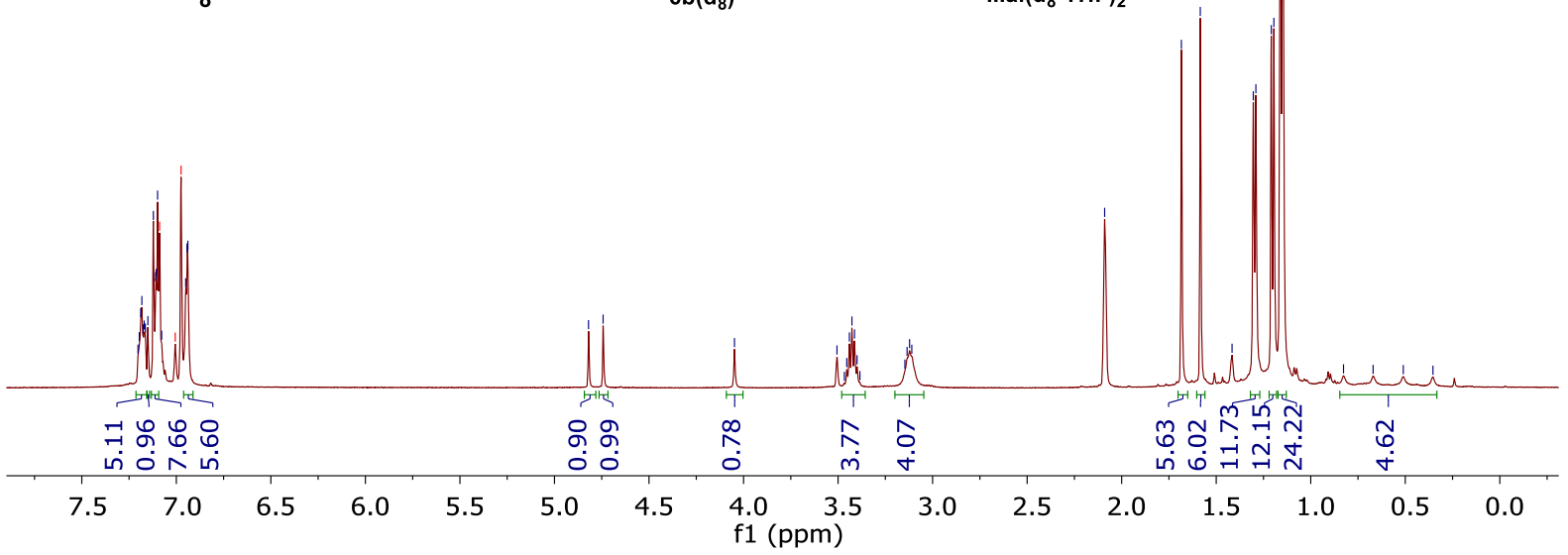

Figure S30: ${ }^{1} \mathrm{H}$ NMR spectrum $\left(500 \mathrm{MHz}, 298 \mathrm{~K}, \mathrm{~d}_{8}\right.$-toluene $+\mathrm{d}_{8}$-THF) resulting from the addition of $d_{8}$-THF $(0.1 \mathrm{ml})$ to a $d_{8}$-toluene solution/suspension of compound 8 . 


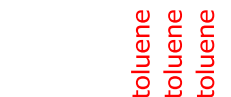

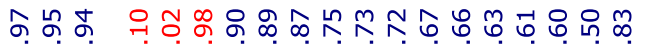
NNN N

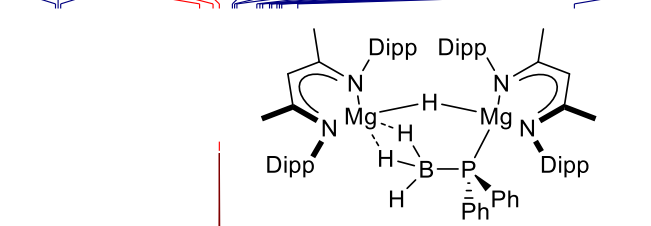

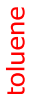

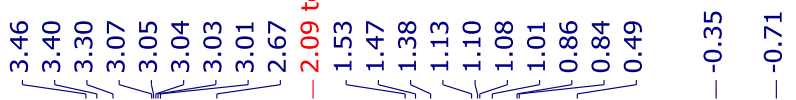

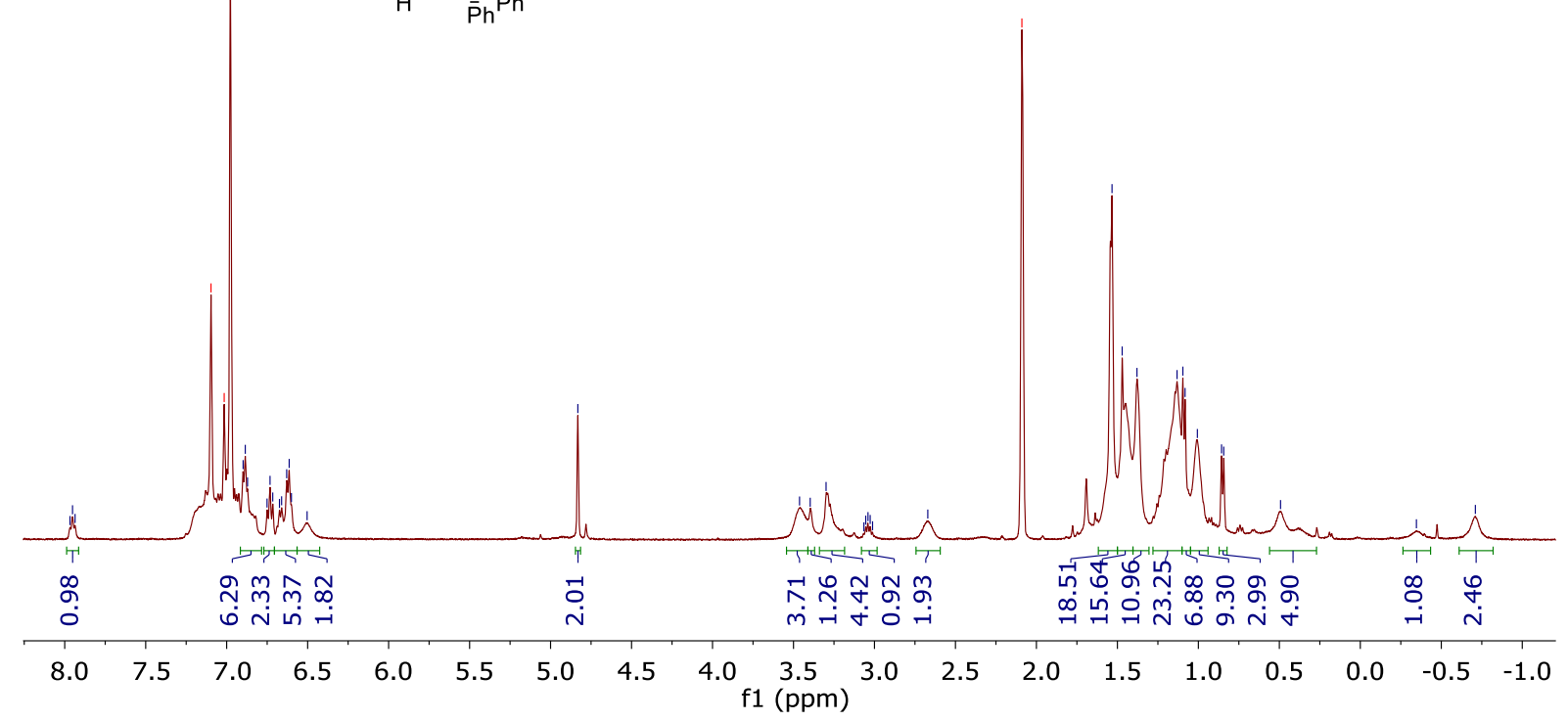

Figure S31: ${ }^{1} \mathrm{H}$ NMR spectrum ( $500 \mathrm{MHz}, 298 \mathrm{~K}, \mathrm{~d}_{8}$-toluene) of compound 8.

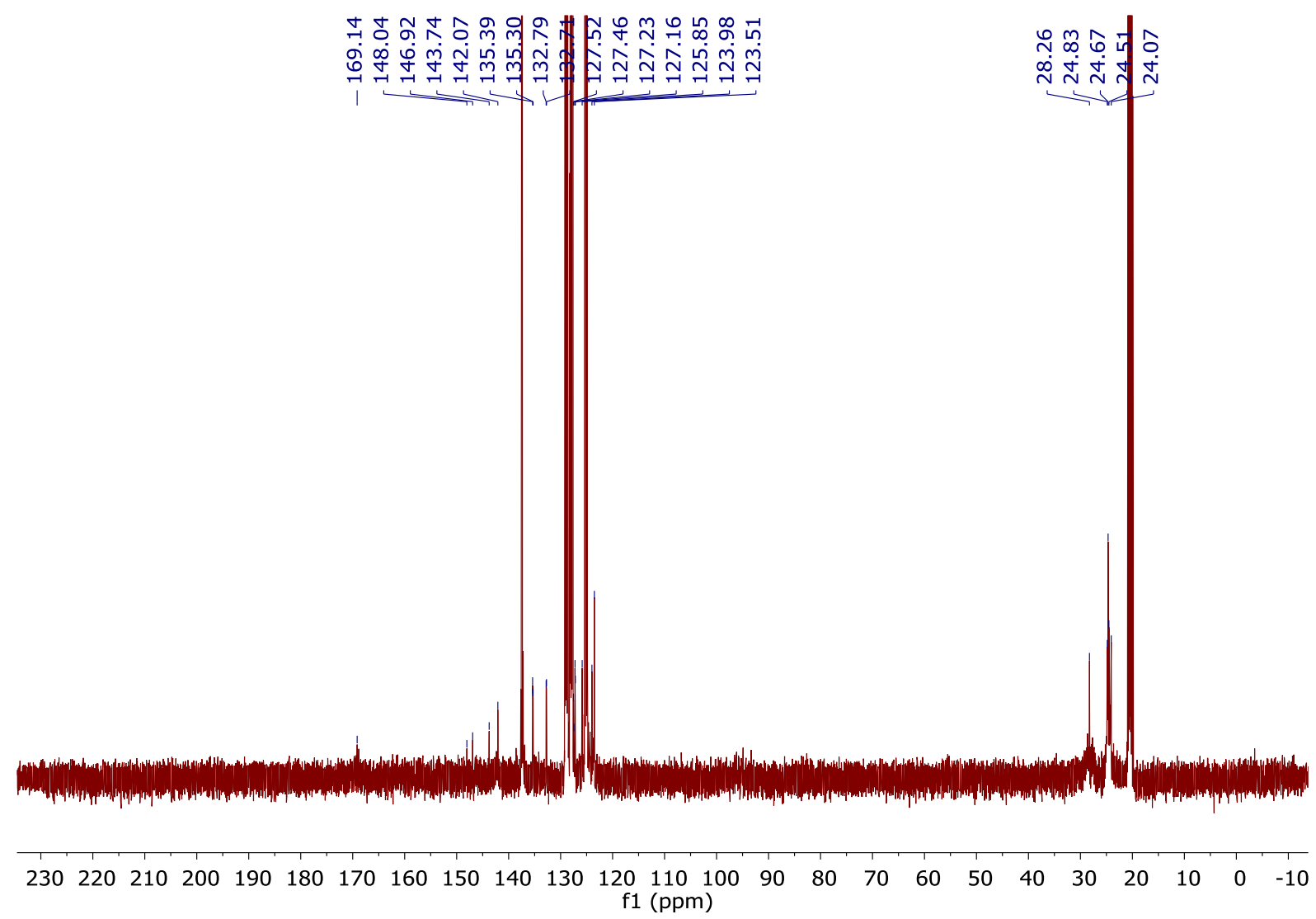

Figure S32: ${ }^{31} \mathrm{C}\left\{{ }^{1} \mathrm{H}\right\}$ NMR spectrum (126 MHz, $298 \mathrm{~K}, \mathrm{~d}_{8}$-toluene) of compound 8. 


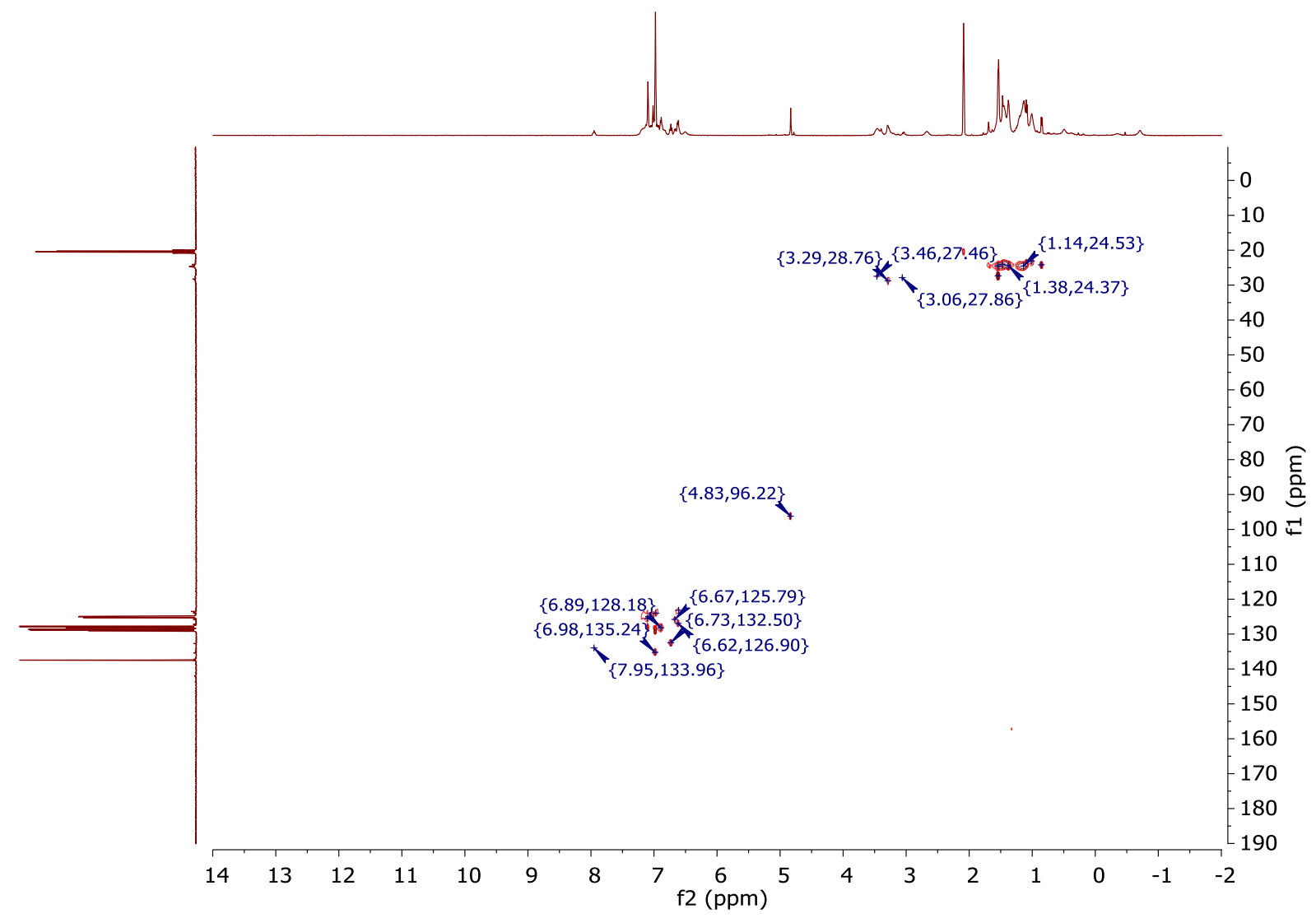

Figure S33: ${ }^{13} \mathrm{C}-{ }^{-1} \mathrm{H}$ HSQC NMR spectrum ( $298 \mathrm{~K}$, d $\mathrm{d}_{8}$-toluene) of compound 8.

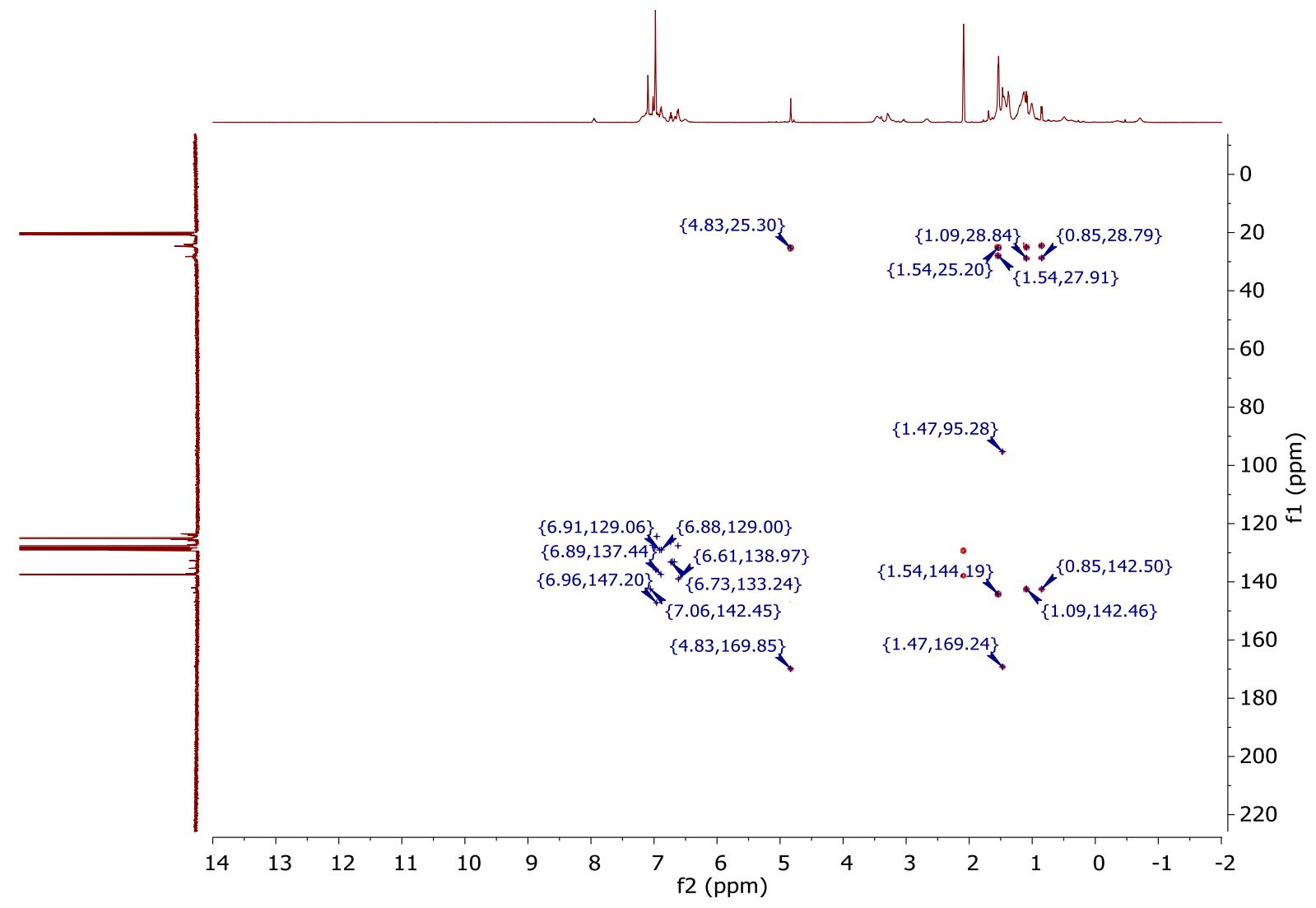

Figure S34: ${ }^{13} \mathrm{C}-{ }^{1} \mathrm{H}$ HMBC NMR spectrum (298 K, d $\mathrm{d}_{8}$-toluene) of compound 8. 


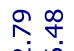 \\ î}

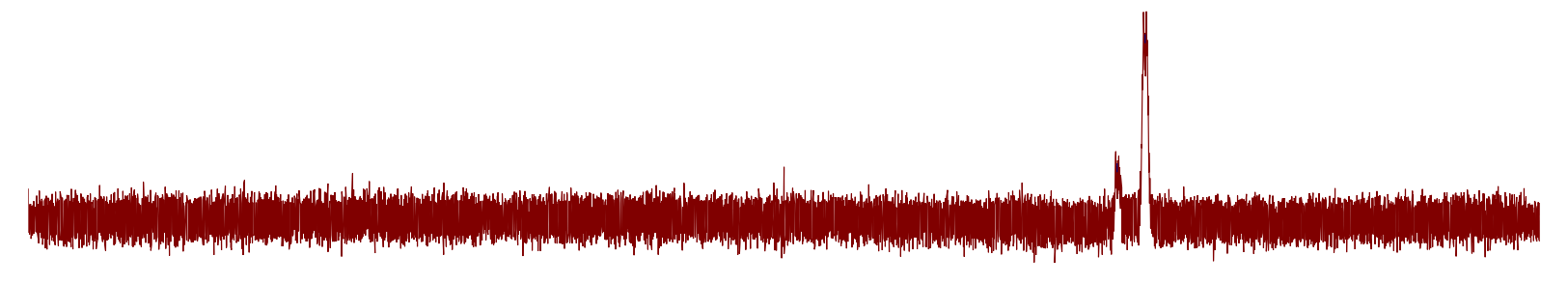

$\begin{array}{llllllllllllllllllll}00 & 90 & 80 & 70 & 60 & 50 & 40 & 30 & 20 & 10 \underset{\mathrm{f} 1(\mathrm{ppm})}{0} & -10 & -20 & -30 & -40 & -50 & -60 & -70 & -80 & -90\end{array}$

Figure S35: ${ }^{31} \mathrm{P}\left\{{ }^{1} \mathrm{H}\right\}$ NMR spectrum (202 MHz, $298 \mathrm{~K}, \mathrm{~d}_{8}$-toluene) of compound 8.

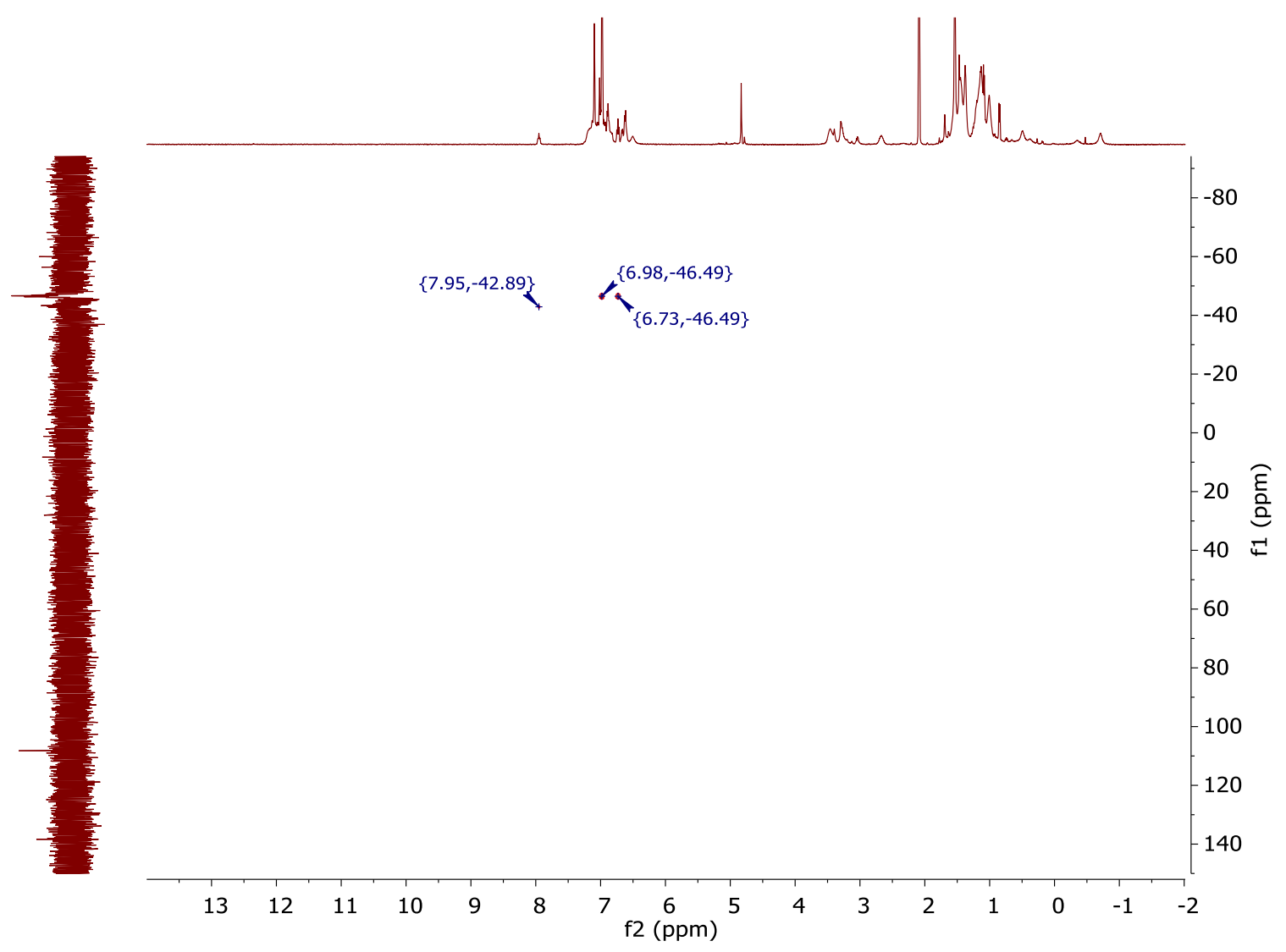

Figure S36: ${ }^{31} \mathrm{P}^{-1} \mathrm{H}$ HMBC NMR spectrum (298 K, d 8 -toluene) of compound 8. 


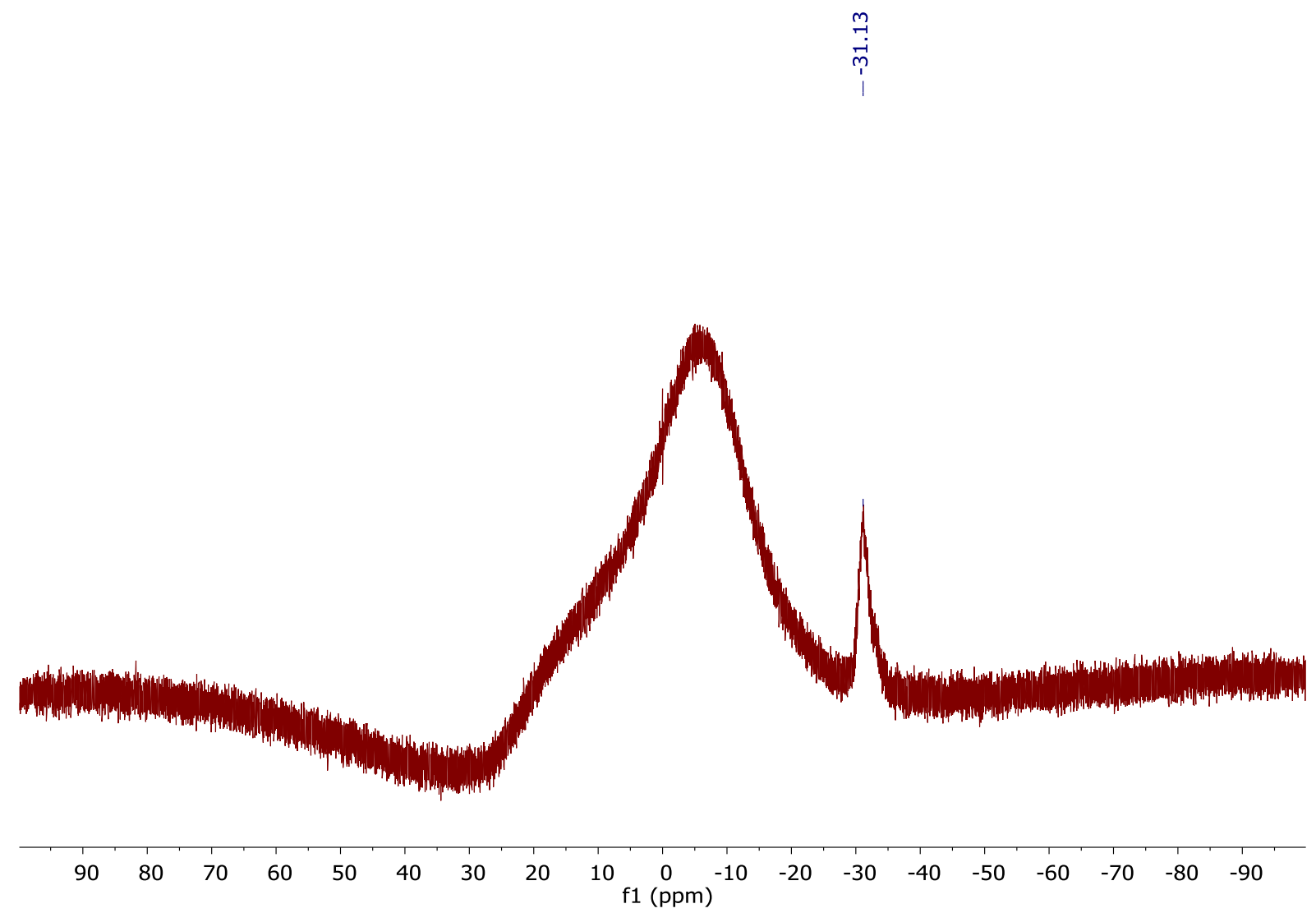

Figure S37: ${ }^{11} \mathrm{~B}$ NMR spectrum ( $160 \mathrm{MHz}, 298 \mathrm{~K}$, $\mathrm{d}_{8}$-toluene) of compound 8.

Compound 9 


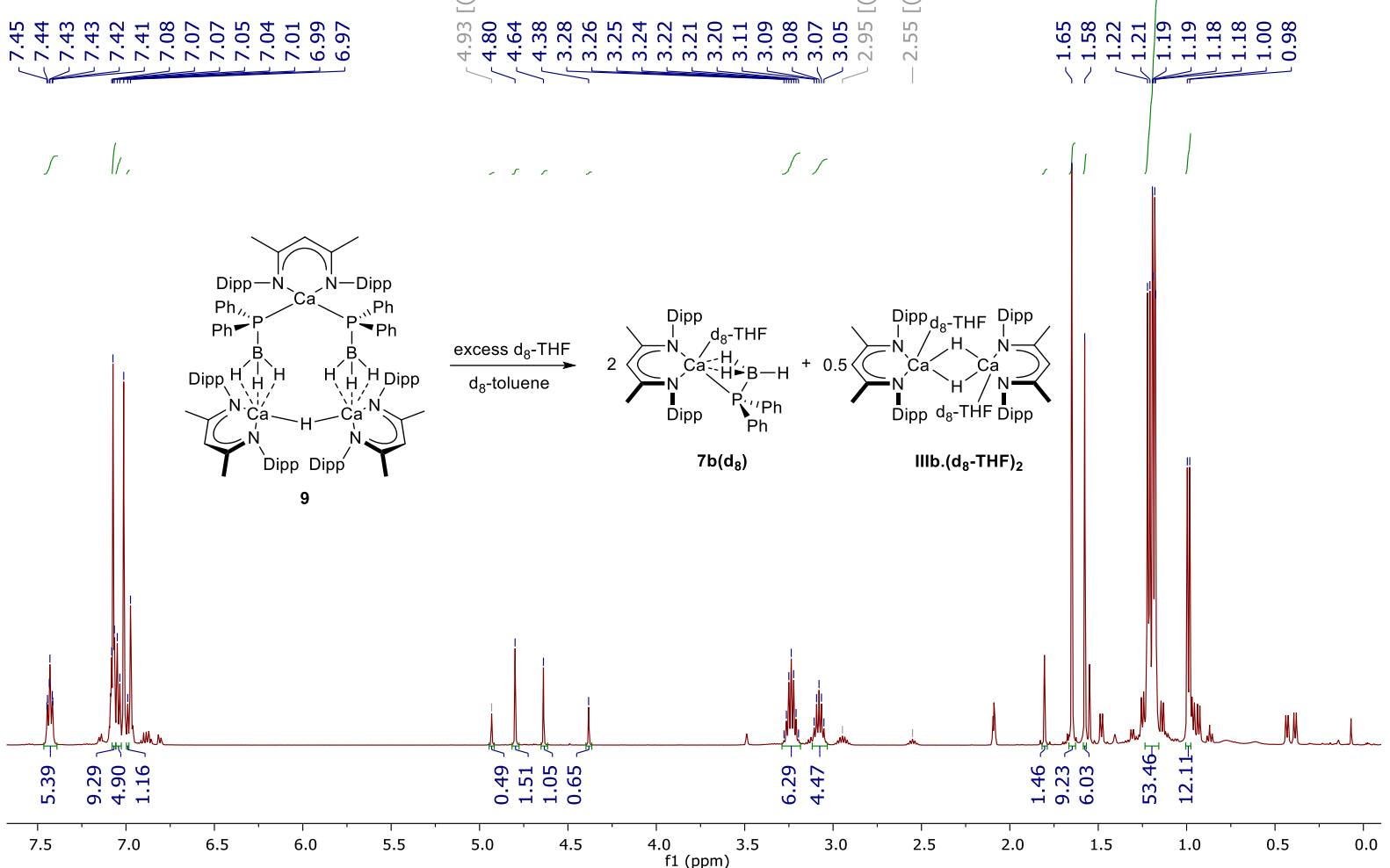

Figure S38: ${ }^{1 H} \mathrm{HMR}$ spectrum $\left(500 \mathrm{MHz}, 298 \mathrm{~K}\right.$, $\mathrm{d}_{8}$-toluene $+\mathrm{d}_{8}$-THF) resulting from the addition of $d_{8}$-THF $(0.05 \mathrm{ml})$ to a $d_{8}$-toluene solution/suspension of compound 9 to produce compounds $7 b\left(d_{8}\right)$ and IIIb.($\left.d_{8}-T H F\right)_{2}$ in situ. Marked peaks correspond to the homoleptic species $\left[(\mathrm{BDI})_{2} \mathrm{Ca}\right]$, resulting from ligand redistribution during high temperature NMR experiments. 


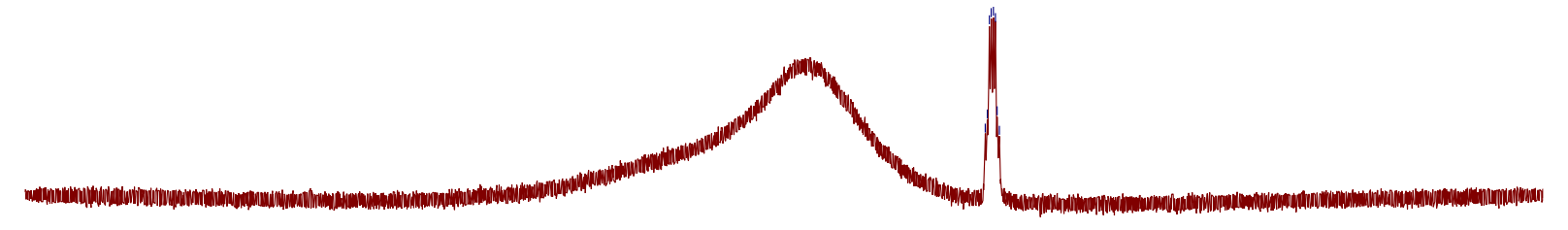

$90 \quad 80$

(7)

60

$50 \quad 40$

20

$10 \stackrel{0}{\mathrm{f} 1}(\mathrm{ppm})$

$-10$

Figure S39: ${ }^{11} \mathrm{~B}$ NMR spectrum $\left(160 \mathrm{MHz}, 298 \mathrm{~K}, \mathrm{~d}_{8}\right.$-toluene $\left.+\mathrm{d}_{8}-\mathrm{THF}\right)$ resulting from the addition of $d_{8}$-THF $(0.05 \mathrm{ml})$ to a $d_{8}$-toluene solution/suspension of compound 9 to produce compounds $7 \mathrm{~b}\left(\mathbf{d}_{8}\right)$ and IIIb. $\left(\mathbf{d}_{8}-\mathrm{THF}\right)_{2}$ in situ. 


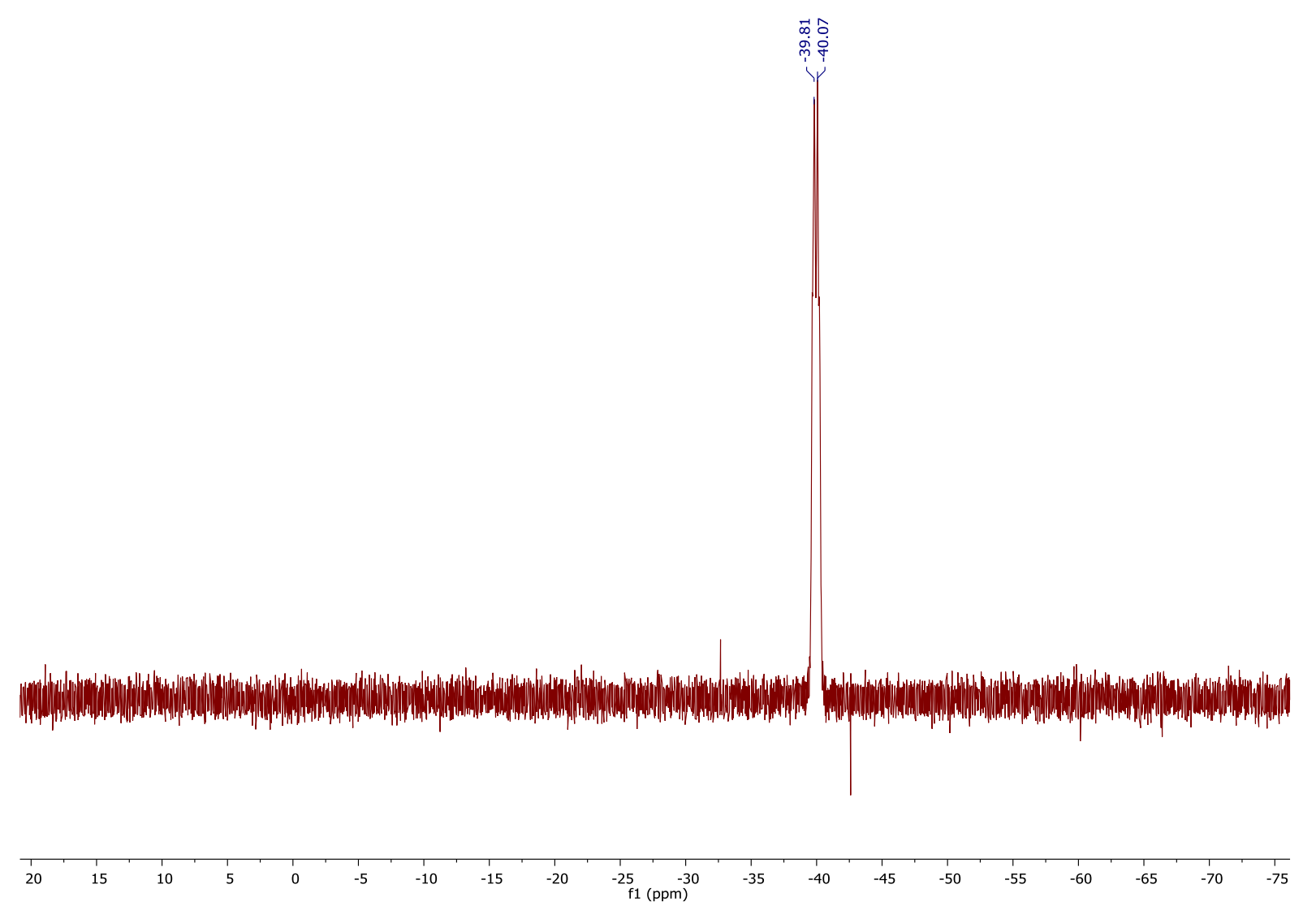

Figure S40: ${ }^{31} \mathrm{P}\left\{{ }^{1} \mathrm{H}\right\}$ NMR spectrum $\left(202 \mathrm{MHz}, 298 \mathrm{~K}, \mathrm{~d}_{8}\right.$-toluene $+\mathrm{d}_{8}$-THF) resulting from the addition of $\mathrm{d}_{8}$-THF $(0.05 \mathrm{ml})$ to a $\mathrm{d}_{8}$-toluene solution/suspension of compound 9 to produce compounds $7 \mathbf{b}\left(\mathbf{d}_{8}\right)$ and IIIb.( $\left.\mathbf{d}_{8}-\mathrm{THF}\right)_{2}$ in situ.

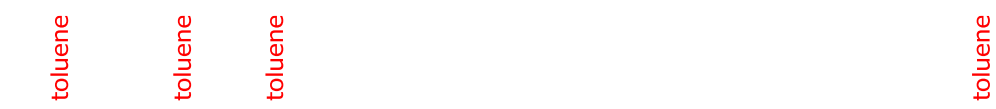

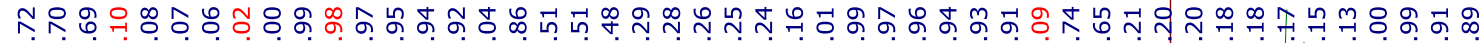

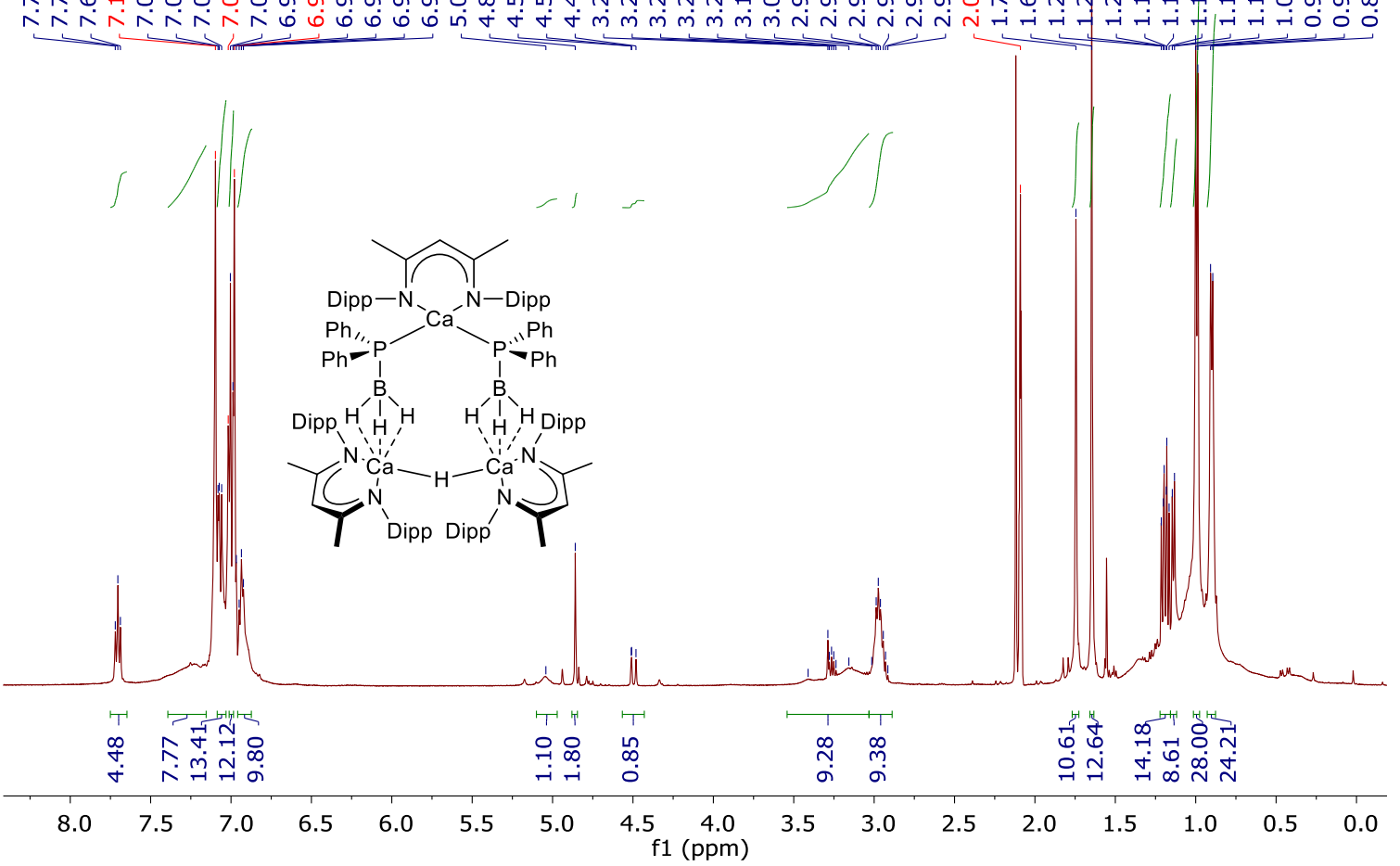

Figure S41: ${ }^{1} \mathrm{H}$ NMR spectrum (500 MHz, $298 \mathrm{~K}$, d8-toluene) of compound 9. 


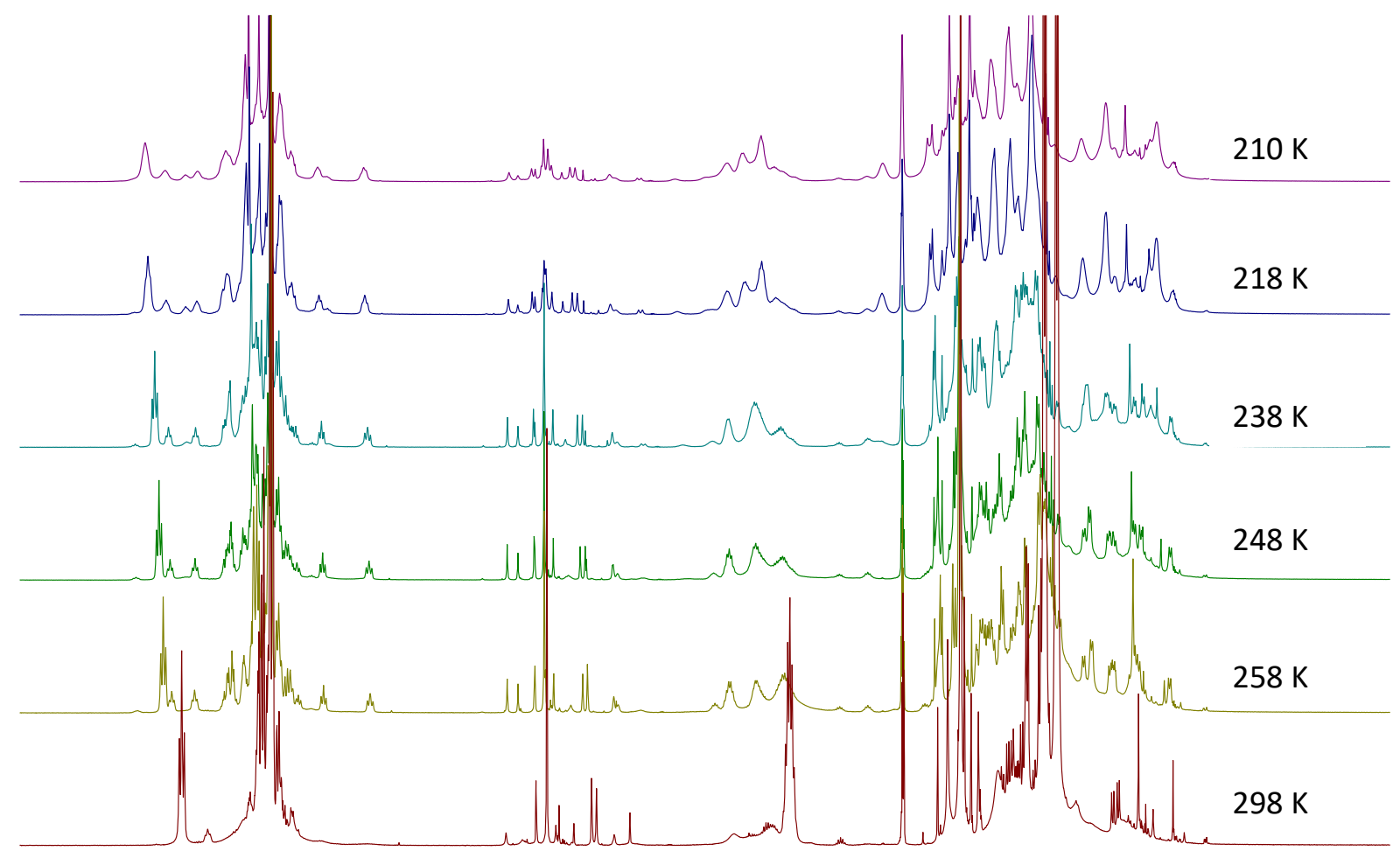

$\begin{array}{lllllllllllllllllllll}8.5 & 8.0 & 7.5 & 7.0 & 6.5 & 6.0 & 5.5 & 5.0 & 4.5 & \underset{\mathrm{f} 1(\mathrm{ppm})}{4.0} & \begin{array}{c}3.5 \\ \mathrm{fmm}\end{array} & 2.0 & 2.5 & 2.0 & 1.5 & 1.0 & 0.5 & 0.0 & -0.5 & -1.0 & -1.5\end{array}$

Figure S42: ${ }^{1} \mathrm{H}$ NMR spectra ( $\mathrm{d}_{8}$-toluene, $400 \mathrm{MHz}$ ) of compound 9 at temperatures between $210 \mathrm{~K}$ and $298 \mathrm{~K}$. 


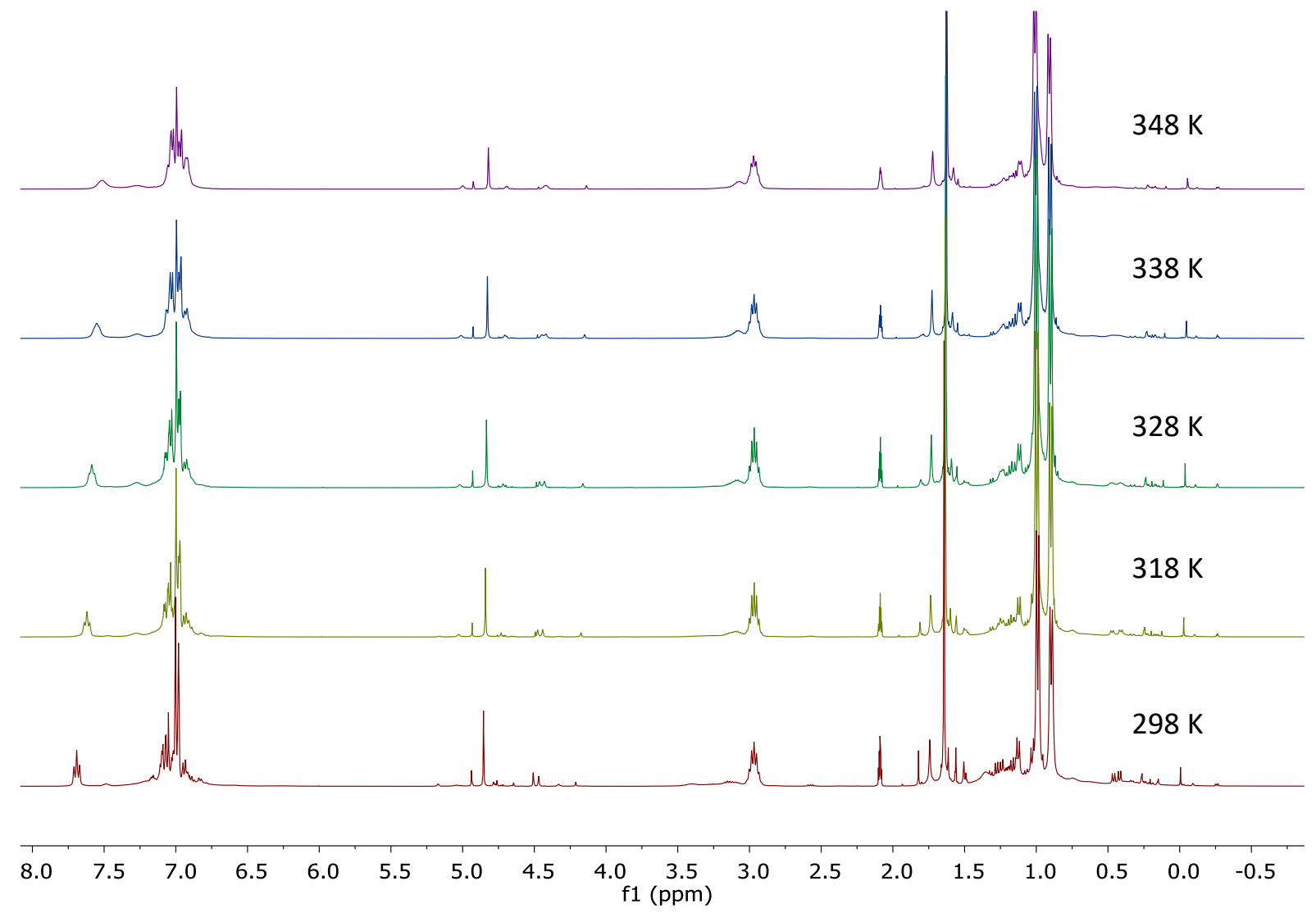

Figure S43: ${ }^{1} \mathrm{H}$ NMR spectra ( $\mathrm{d}_{8}$-toluene, $400 \mathrm{MHz}$ ) of compound 9 at temperatures between $298 \mathrm{~K}$ and $348 \mathrm{~K}$.

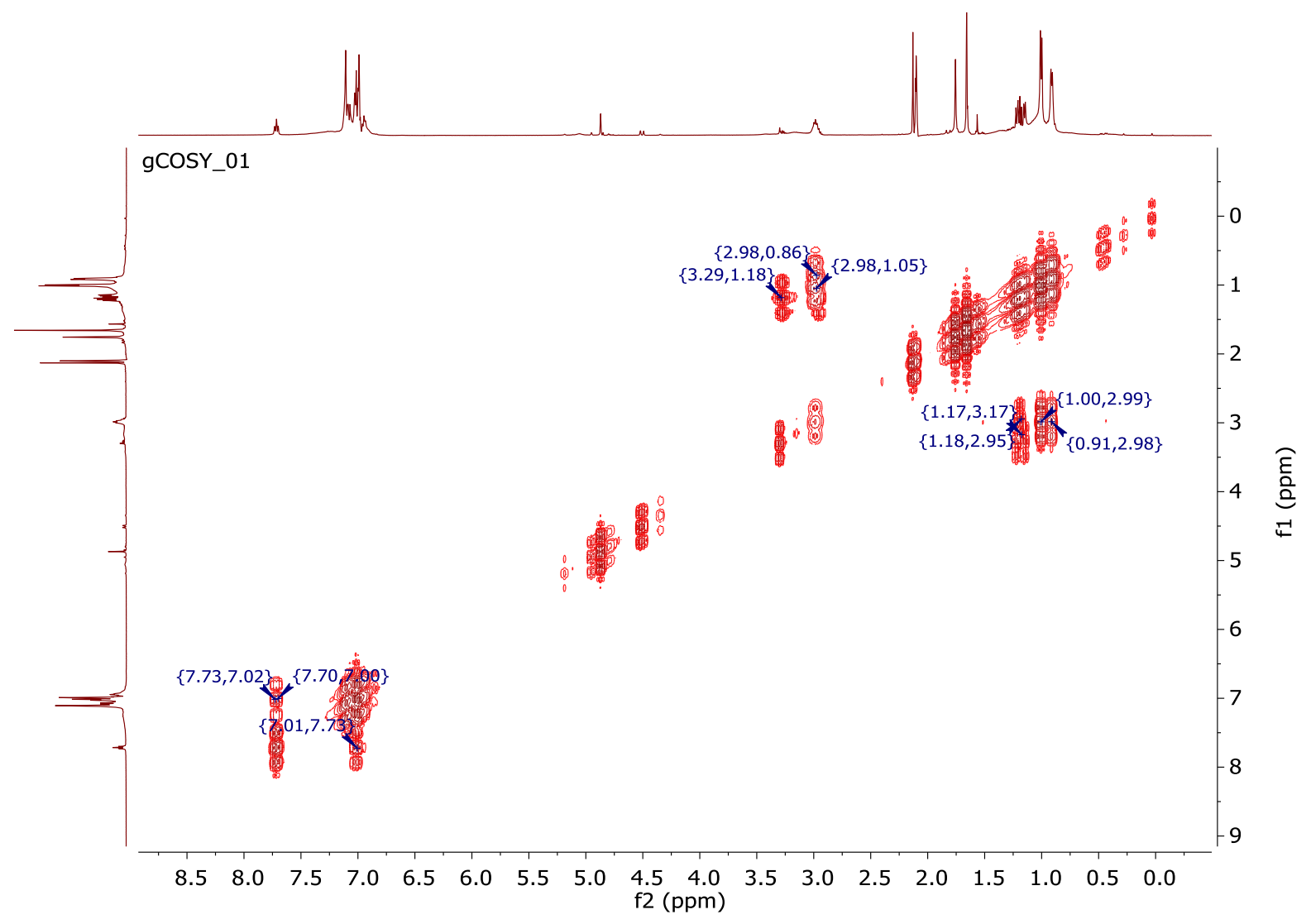

Figure 44: ${ }^{1} \mathrm{H}-{ }^{1} \mathrm{H}$ COSY NMR spectrum ( $298 \mathrm{~K}$, $\mathrm{d}_{8}$-toluene) of compound 9. 


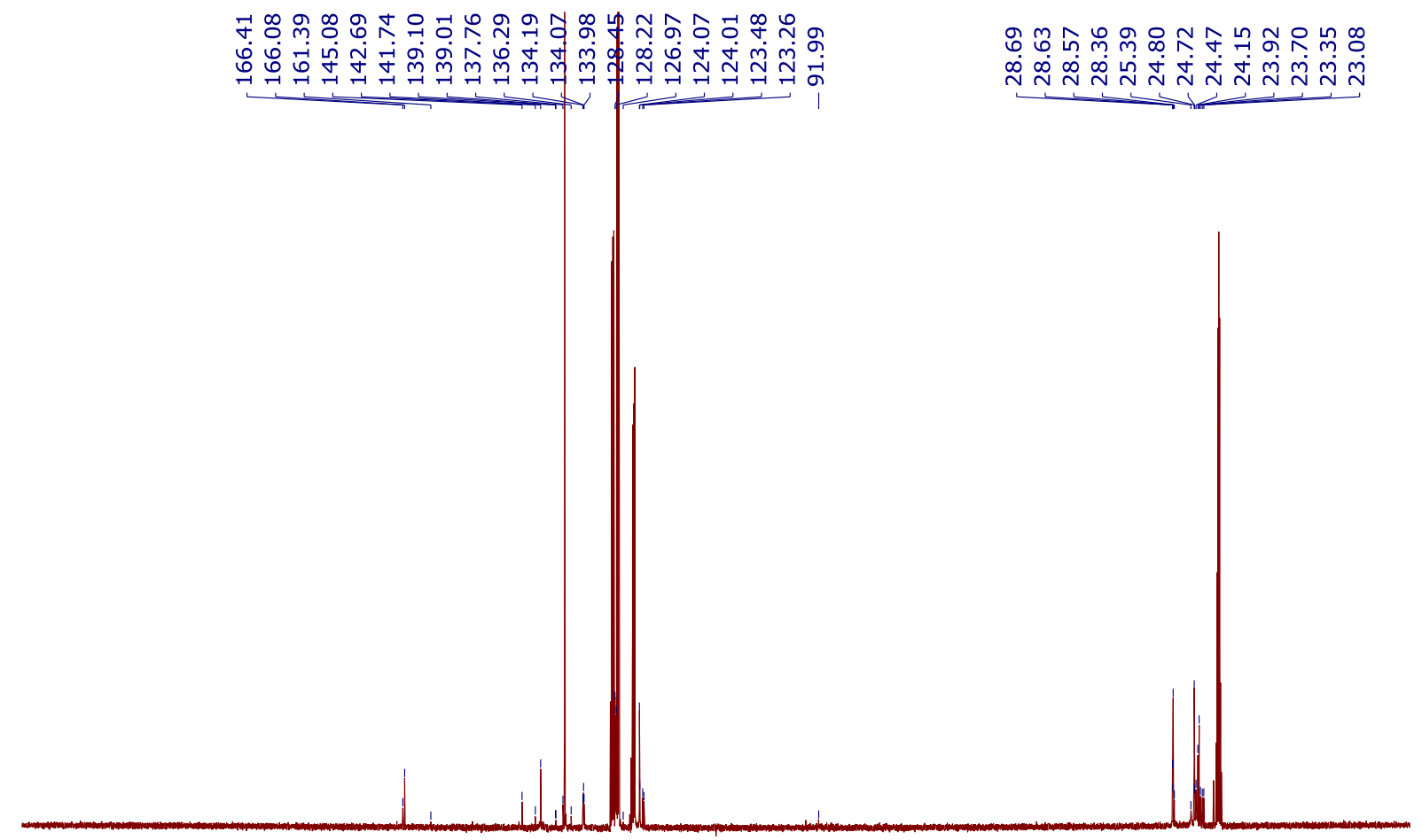

$\begin{array}{lllllllllllllllllllllllll}230 & 220 & 210 & 200 & 190 & 180 & 170 & 160 & 150 & 140 & 130 & \begin{array}{c}120 \\ \mathrm{f} 1\end{array} \begin{array}{l}110 \\ (\mathrm{ppm})\end{array} & 100 & 90 & 80 & 70 & 60 & 50 & 40 & 30 & 20 & 10 & 0 & -10\end{array}$

Figure S45: ${ }^{31} \mathrm{C}\left\{{ }^{1} \mathrm{H}\right\}$ NMR spectrum (126 MHz, $298 \mathrm{~K}, \mathrm{~d}_{8}$-toluene) of compound 9.

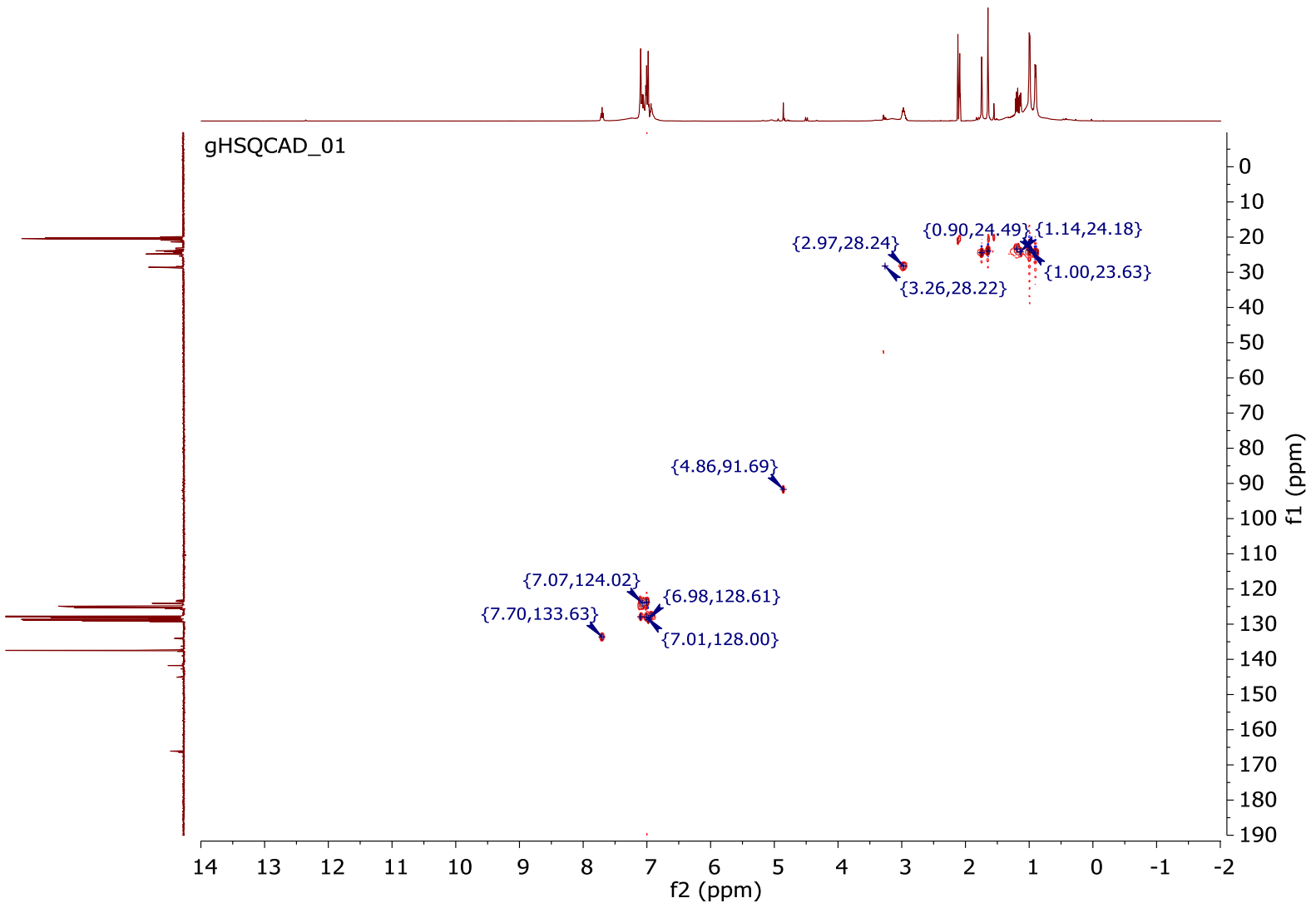

Figure S46: ${ }^{13} \mathrm{C}-{ }^{1} \mathrm{H}$ HSQC NMR spectrum (298 K, d8-toluene) of compound 9. 


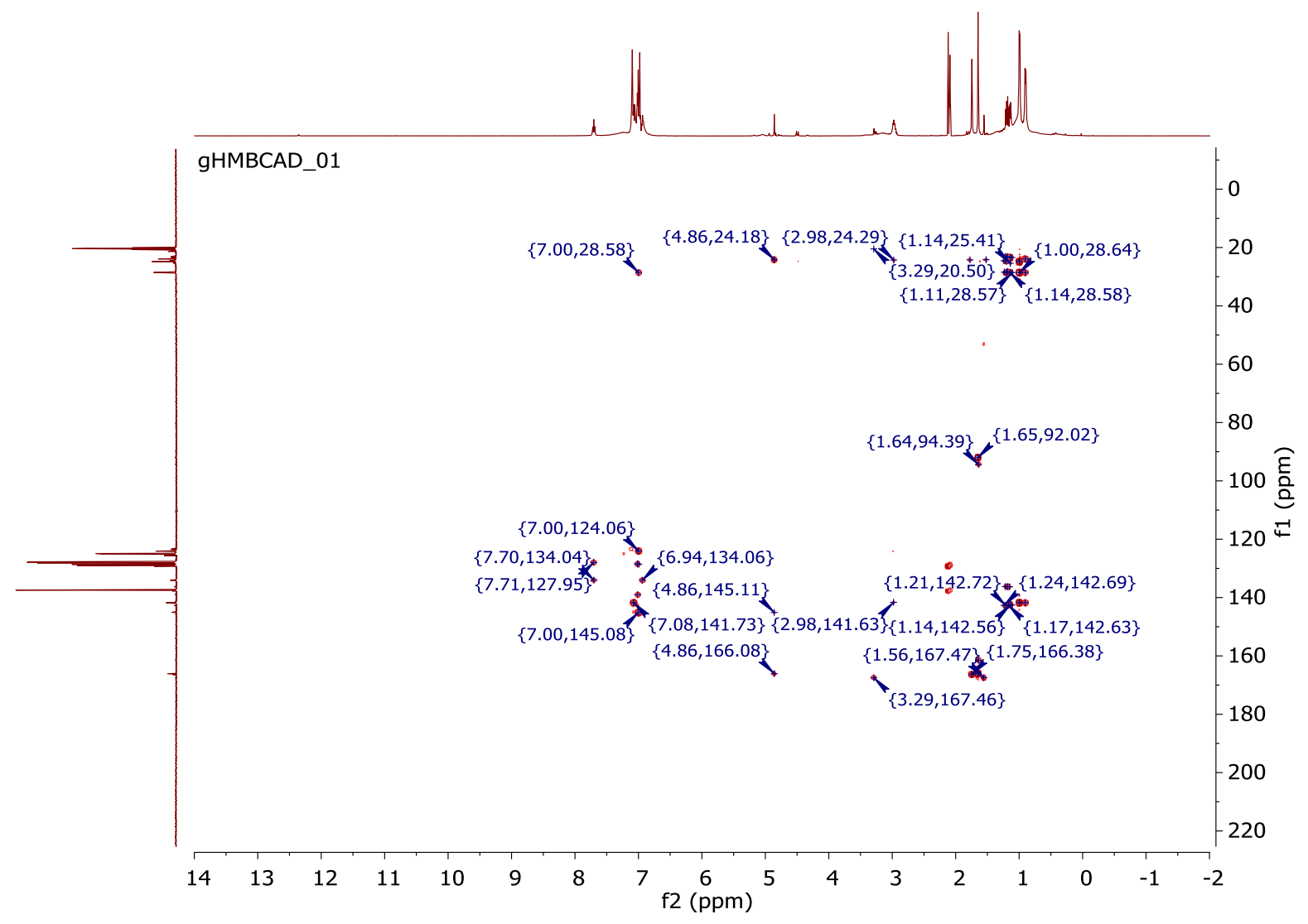

Figure S47: ${ }^{13} \mathrm{C}-{ }^{-1} \mathrm{H}$ HMBC NMR spectrum (298 K, $\mathrm{d}_{8}$-toluene) of compound 9.

$$
\text { 角 }
$$

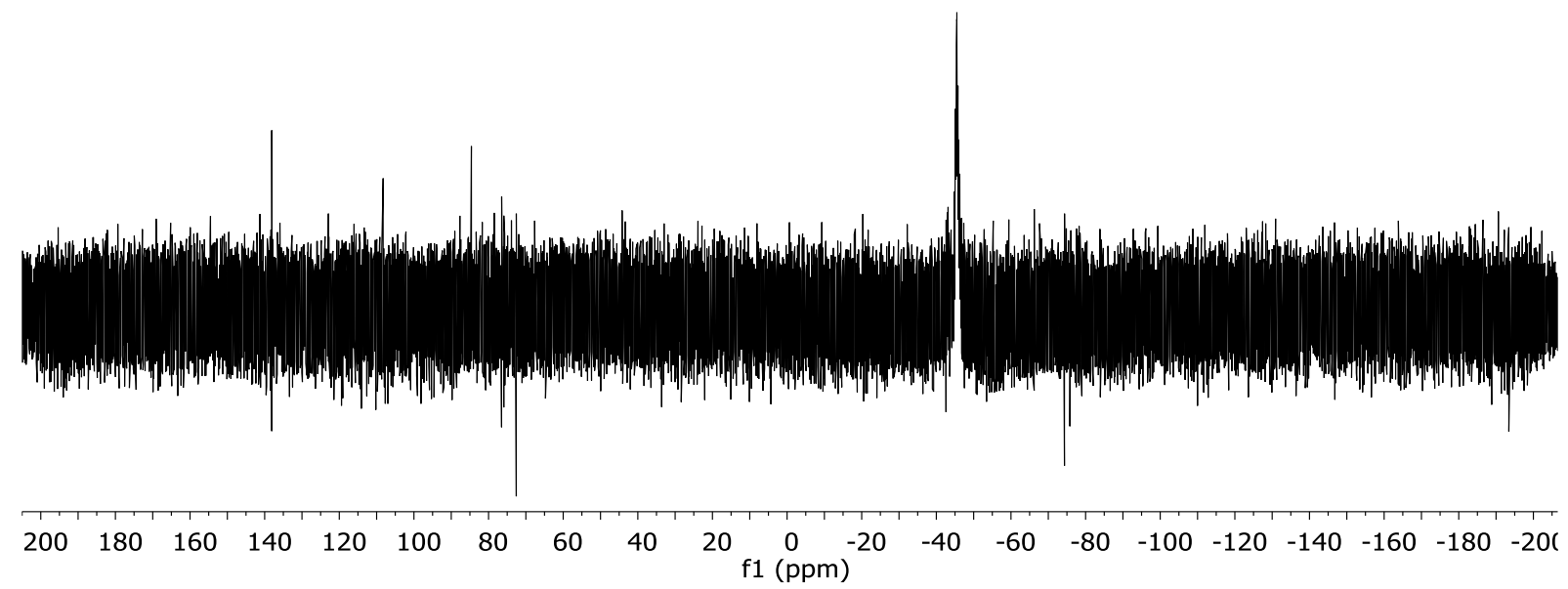

Figure S48: ${ }^{31} \mathrm{P}\left\{{ }^{1} \mathrm{H}\right\}$ NMR spectrum (202 MHz, $298 \mathrm{~K}$, d8-toluene) of compound 9. 


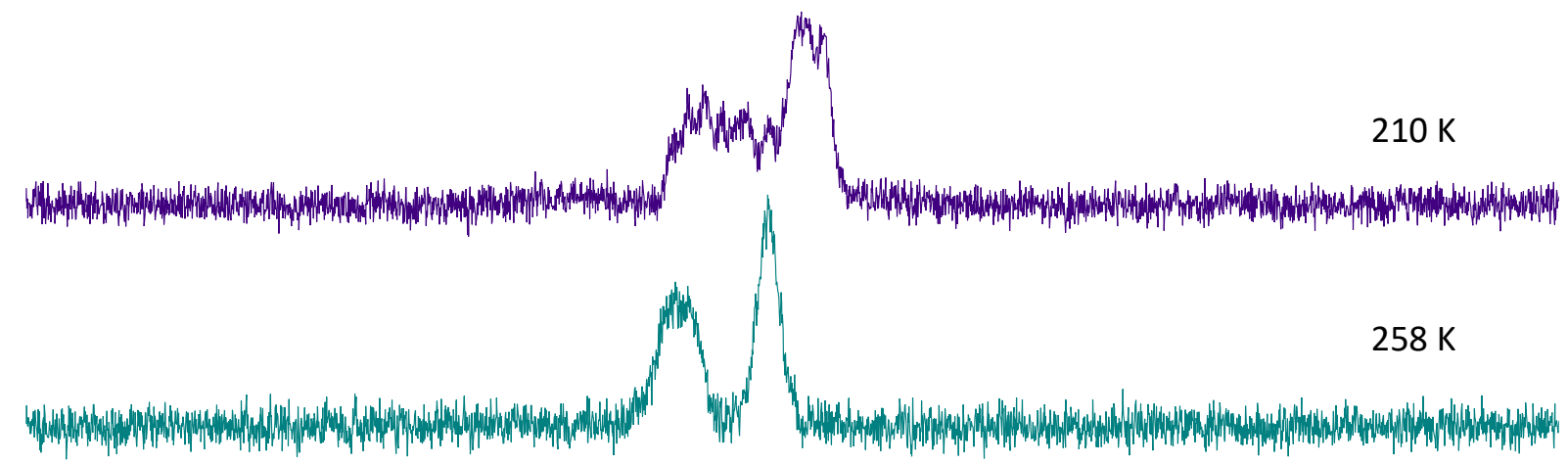

Wy.t.

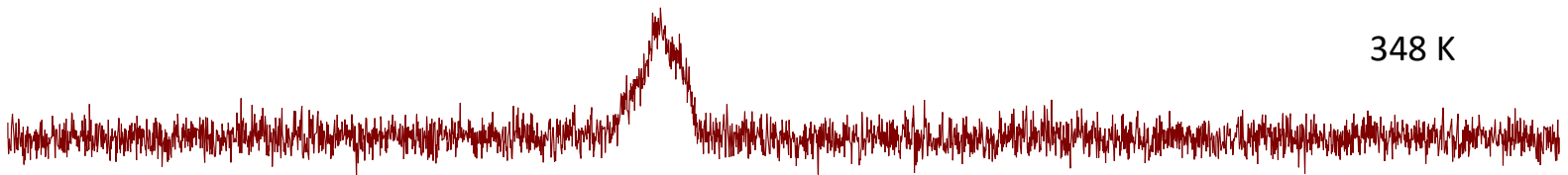

$\begin{array}{lllllllllllllllllll}-29 & -31 & -33 & -35 & -37 & -39 & -41 & -43 & -45 & -47 & -49 & -51 & -53 & -55 & -57 & -59 & -61 & -63 & -65\end{array}$

Figure S49: ${ }^{31} \mathrm{P}\left\{{ }^{1} \mathrm{H}\right\}$ NMR spectra (162 MHz, $\mathrm{d}_{8}$-toluene) of compound 9 at temperatures from $210 \mathrm{~K}$ to $348 \mathrm{~K}$.

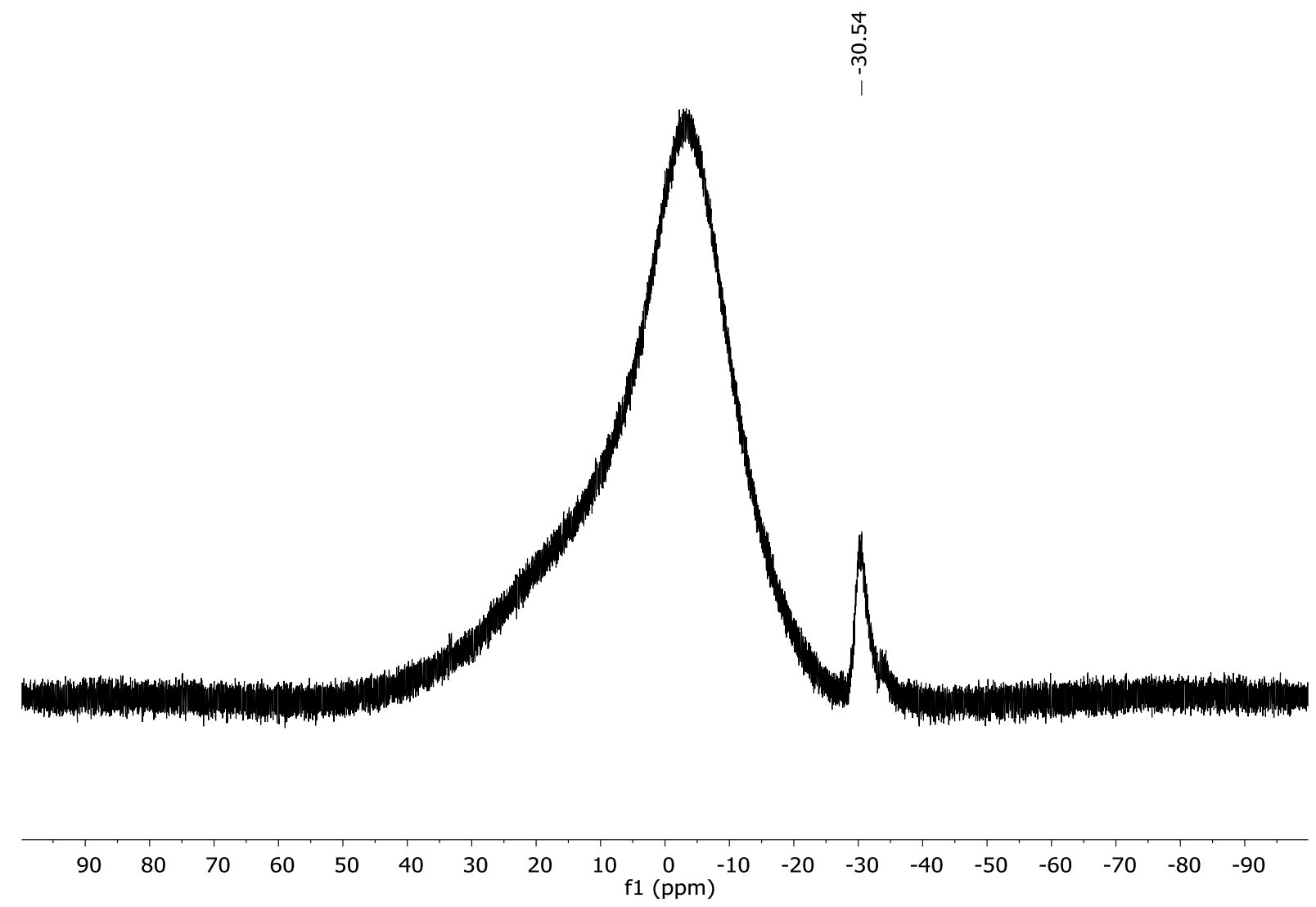

Figure S50: ${ }^{11} \mathrm{~B}$ NMR spectrum ( $160 \mathrm{MHz}, 298 \mathrm{~K}$, $\mathrm{d}_{8}$-toluene) of compound 9. 


\section{Compound 10}

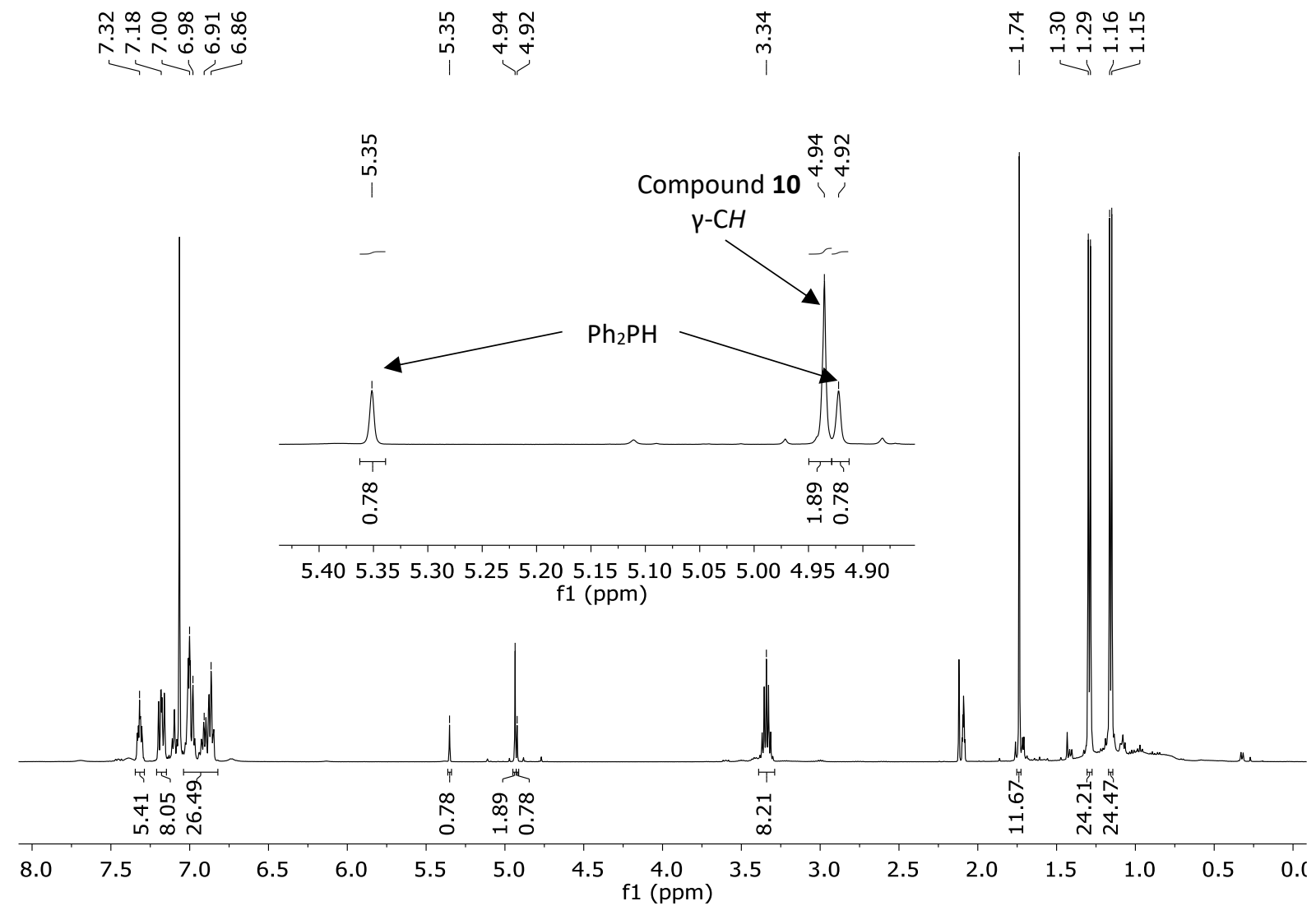

Figure S51: in situ ${ }^{1} \mathrm{H}$ NMR spectrum (500 MHz, $298 \mathrm{~K}, \mathrm{~d}_{8}$-toluene) of the reaction between compound 6a with two equivalents of $\mathrm{Ph}_{2} \mathrm{PH} \cdot \mathrm{BH}_{3}$. Inset: expanded spectrum to show resonances of $\mathrm{Y}-\mathrm{CH}$ and $\mathrm{Ph}_{2} \mathrm{PH}$ protons.

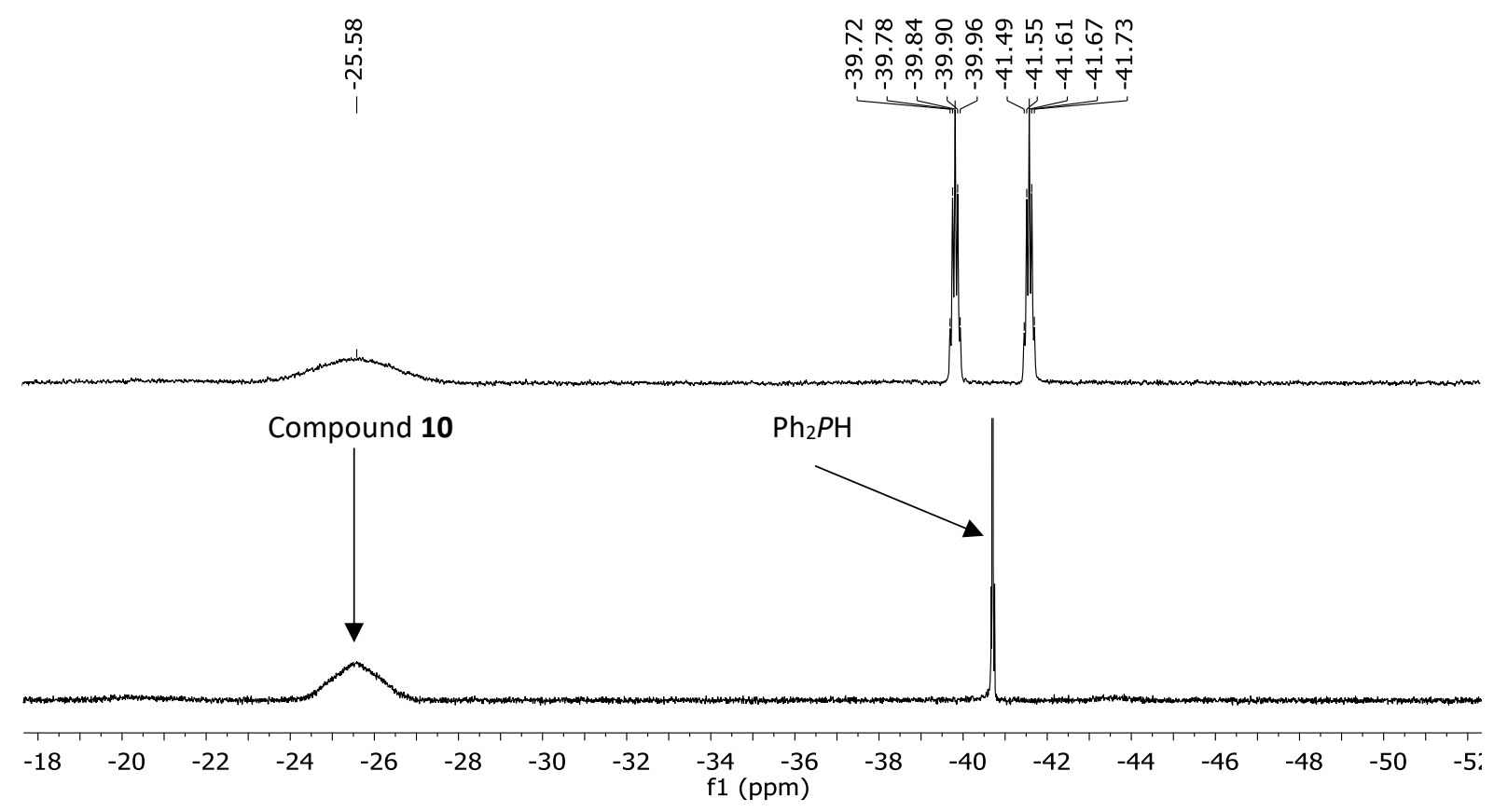

Figure S52: Expanded in situ ${ }^{31} \mathrm{P}$ (top) and ${ }^{31} \mathrm{P}\left\{{ }^{1} \mathrm{H}\right\}$ (bottom) NMR spectra (122 MHz, $298 \mathrm{~K}$, $\mathrm{d}_{8}$-toluene) of the reaction between compound 10 and two equivalents of $\mathrm{Ph}_{2} \mathrm{PH} \cdot \mathrm{BH}_{3}$. 


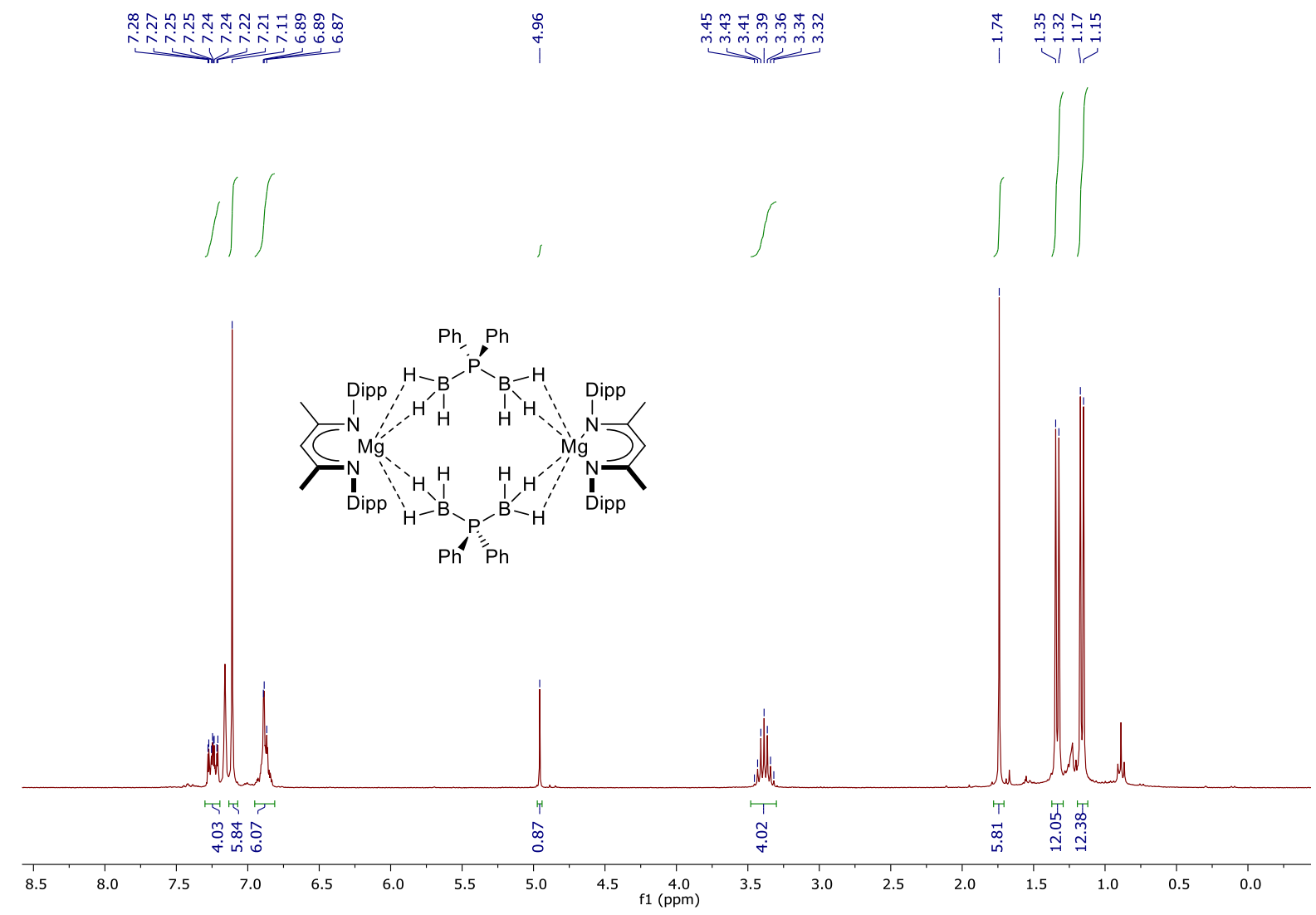

Figure S53: ${ }^{1} \mathrm{H}$ NMR spectrum of compound 10 (300 MHz, $\left.\mathrm{C}_{6} \mathrm{D}_{6}, 298 \mathrm{~K}\right)$.

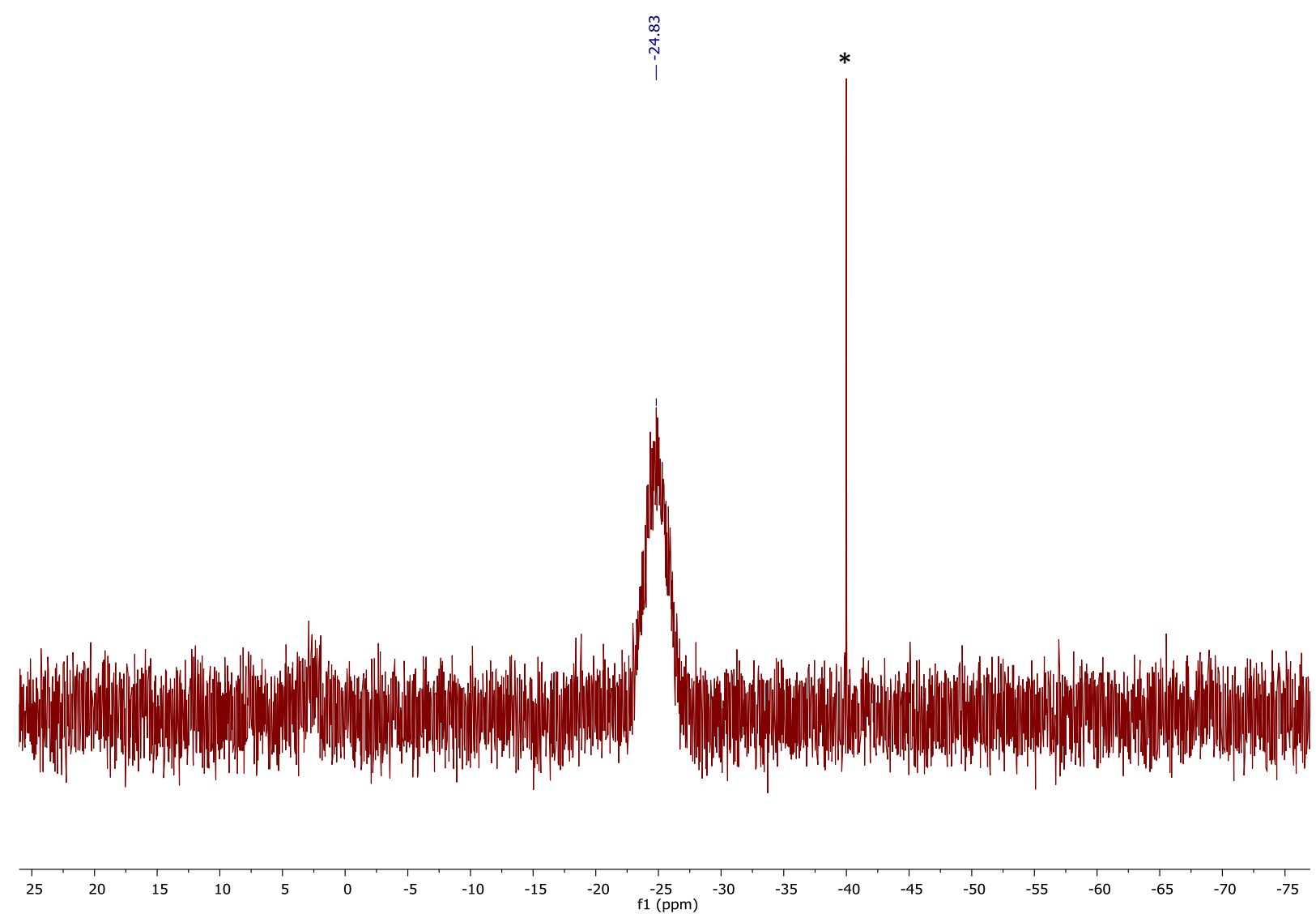

Figure S54: ${ }^{31} \mathrm{P}\left\{{ }^{1} \mathrm{H}\right\}$ NMR spectrum of compound $10\left(122 \mathrm{MHz}, \mathrm{C}_{6} \mathrm{D}_{6}, 298 \mathrm{~K}\right) .{ }^{*}=$ trace $\mathrm{Ph}_{2} \mathrm{PH}$ impurity. 


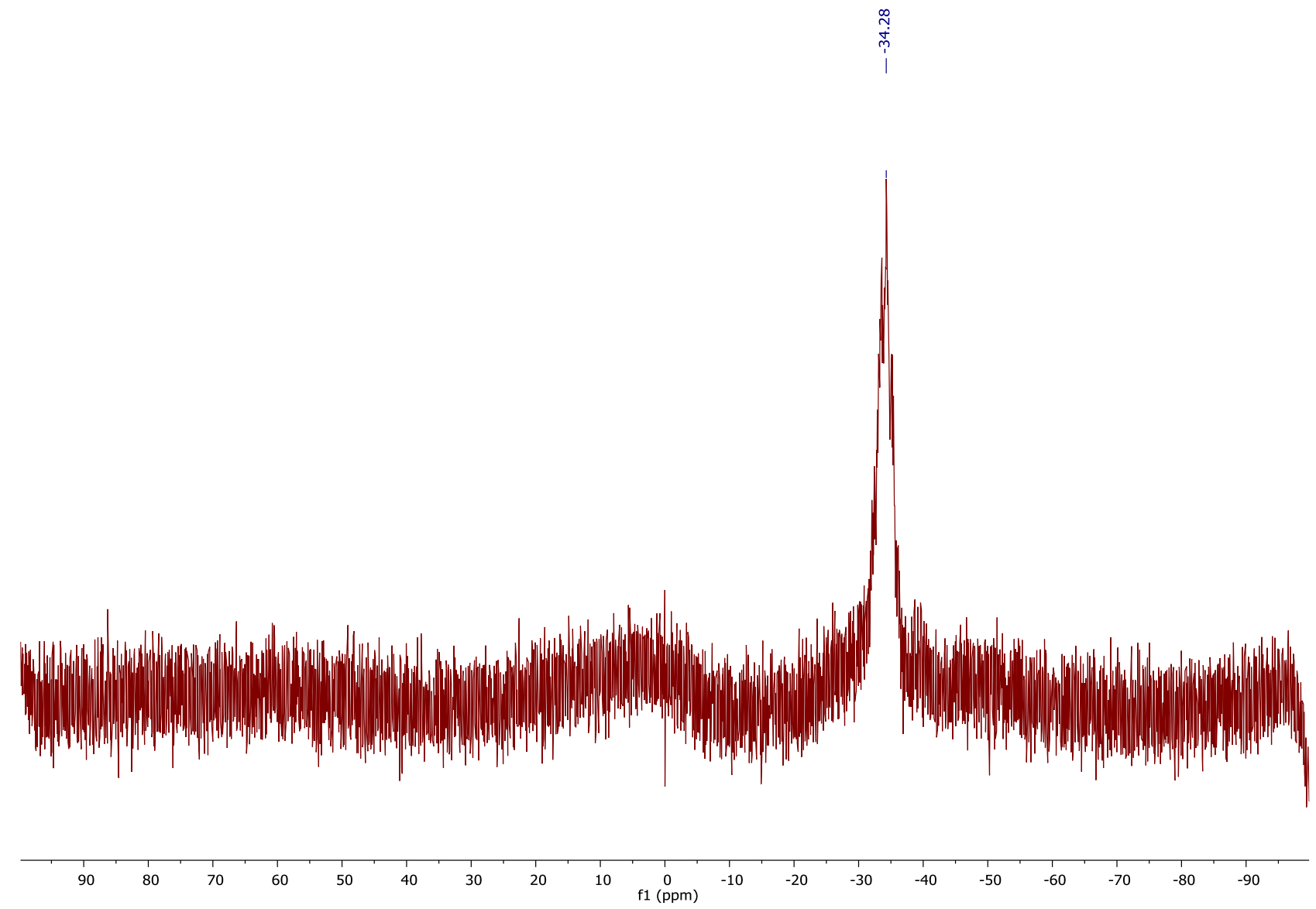

Figure S55: ${ }^{11} \mathrm{~B}$ NMR (96 MHz, $\mathrm{C}_{6} \mathrm{D}_{6}, 298 \mathrm{~K}$ ) of compound 10. 


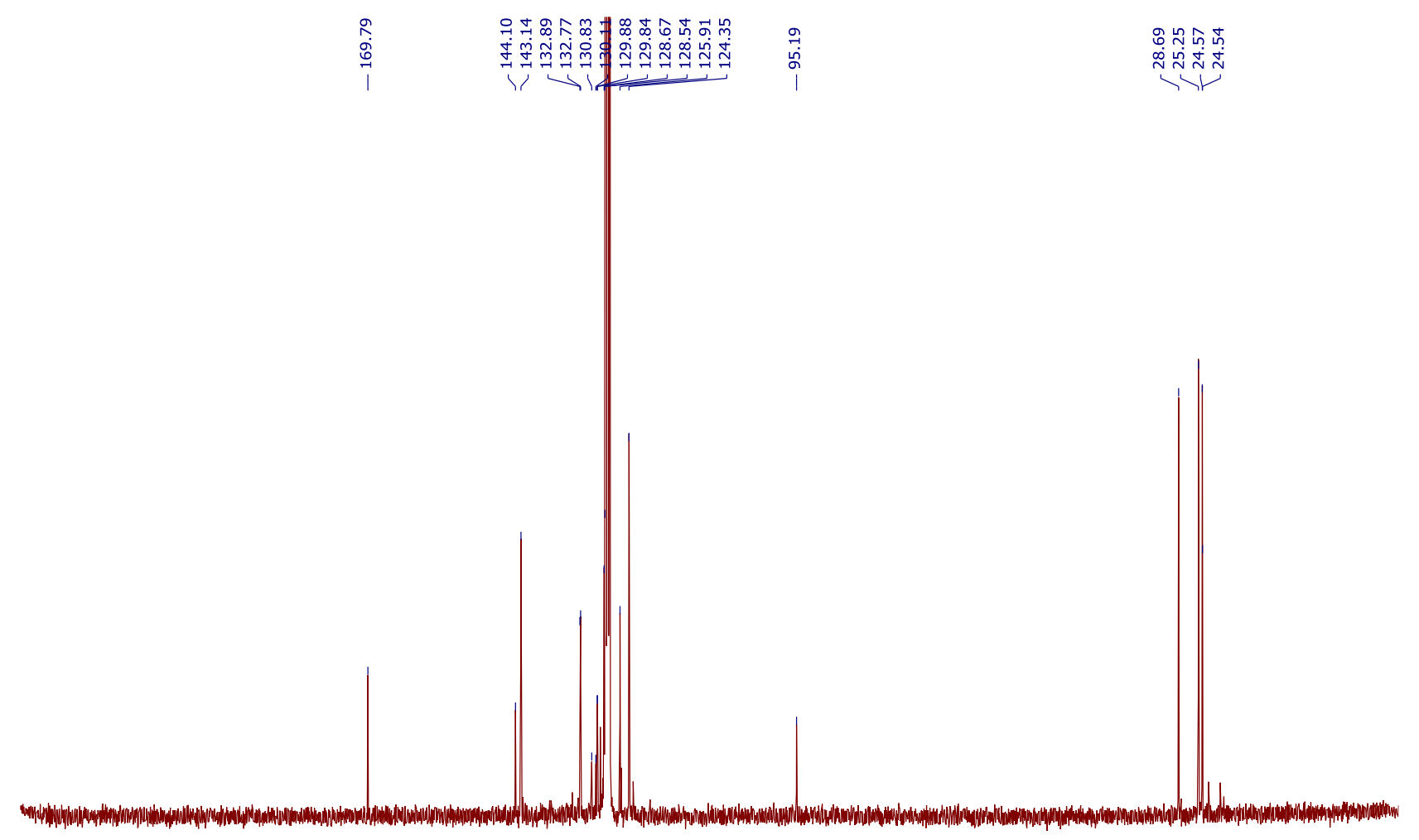

$\begin{array}{llllllllllllllllllllllllllll}30 & 220 & 210 & 200 & 190 & 180 & 170 & 160 & 150 & 140 & 130 & 120 & \begin{array}{l}110 \\ \mathrm{f} 1(\mathrm{ppm})\end{array} & 100 & 90 & 80 & 70 & 60 & 50 & 40 & 30 & 20 & 10 & 0 & \end{array}$

Figure S56: ${ }^{13} \mathrm{C}\left\{{ }^{1} \mathrm{H}\right\}$ NMR $\left(76 \mathrm{MHz}, \mathrm{C}_{6} \mathrm{D}_{6}, 298 \mathrm{~K}\right)$ of compound 10.

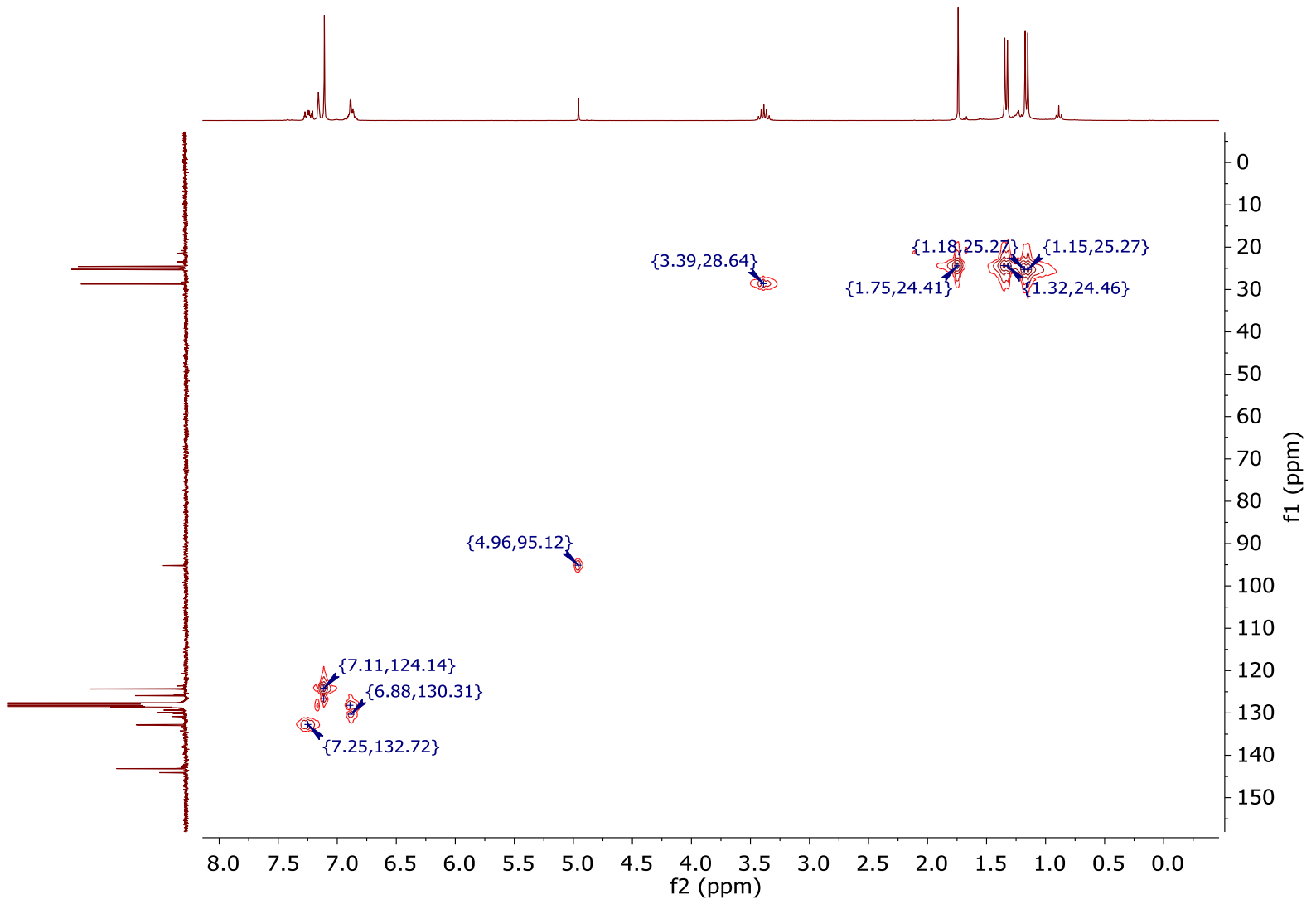

Figure S57: ${ }^{13} \mathrm{C}-{ }^{-1} \mathrm{H}$ HSQC NMR spectrum $\left(\mathrm{C}_{6} \mathrm{D}_{6}, 298 \mathrm{~K}\right)$ of compound 10. 


\section{References}

1. Dolomanov, O. V.; Bourhis, L. J.; Gildea, R. J.; Howard, J. A. K.; Puschmann, H., OLEX2: a complete structure solution, refinement and analysis program. Journal of Applied Crystallography 2009, 42, 339-341.

2. Sheldrick, G. M., SHELXT - Integrated space-group and crystal-structure determination. Acta Crystallographica A-Foundation and Advances 2015, 71, 3-8. 